Copyright is owned by the author of the thesis. Permission is given for a copy to be downloaded by an individual for the purpose of research and private study only. The thesis may not be reproduced elsewhere without the permission of the author. 


\section{Sophisticated Mediators: \\ New Zealand-born Samoan first-time mothers and strategies for their health and wellbeing}

Marianna Ellen Churchward 


\title{
Sophisticated Mediators: \\ New Zealand-born Samoan first-time mothers and strategies for their health and wellbeing
}

\author{
by \\ Marianna Ellen Churchward
}

A thesis

submitted in partial fulfilment of the requirements for the Degree of Master of Arts (Applied) in Social Science Research

Victoria University of Wellington 


\title{
DEDICATION
}

To the most sophisticated 'Sophisticated Mediator' of all my beautiful mother

Anasetasia Maria Tua Churchward (nee Tasi Mafina Tupuola)

\author{
To an emerging 'Sophisticated Mediator' \\ my beautiful daughter \\ Isabella Malia McMurrich Churchward Woods
}

James Patrick Churchward

RIP

$24 / 03 / 70-23 / 08 / 1992$ 


\section{ABSTRACT}

This thesis explores the experiences of four New Zealand-born Samoan first time mothers during pregnancy, childbirth and early motherhood living in Wellington. The impetus for this research arose from findings that showed a considerable variation in the prevalence of postnatal depression between Samoan women (7.6\%), Tongan women (30.9\%) and others (20\% for all New Zealand mothers).

Qualitative, face-to-face individual interviews were conducted within a qualitative feminist framework. The two interviews with each woman were conducted during the last trimester of their pregnancy (28+weeks gestation); and 12 months post-birth. The thesis drew upon the four-element model - Epistemology, Theoretical Perspective, Methodology and Methods to provide a framework to conceptualise and clarify the foundation for this research project. Thus the thesis is best described as a feminist phenomenological social constructionist approach.

The findings revealed the women of this study were 'Sophisticated Mediators' who, although faced with many challenges throughout their pregnancy, childbirth and early motherhood experiences, mediated successfully between, and within, existing cultural and belief systems i.e. Samoan traditional values and New Zealand cultural system; to acquire or maintain resilience toward depressive symptoms during early motherhood. Support structures such as family, in particular mothers, proved to be a vital source of support for the women.

Recommendations arising from the research are targeted at support providers and family members and this is discussed in detail. Antenatal care was important although antenatal classes not so; conversely, the women were high adopters of technology in the form of the internet to access information. It is vital for support providers to recognise the high regard the women had for the internet as a source of valuable and easily accessible information, and utilise those avenues more to provide information that will complement or improve the existing support structures. During the antenatal period, women should be encouraged to develop or strengthen relationships with a significant female who will be with them throughout the childbirth and early motherhood process. 


\section{ACKNOWLEDGEMENTS}

This thesis is not an individual labour of love, but a culmination of the love and support of the wonderful people I am fortunate to have beside me. Firstly, my sincere thanks to the four amazing women who shared their stories with me and whose voices give this thesis life. Your experiences and wisdom are an inspiration to us all.

My ultimate gratitude and love to my parents Tua and Joseph, ever so supportive, ever so reassuring, ever so 'sophisticated' - fa'amalo le tapuai. Thanks for being awesome grandparents to: André, Karlos, Hayley, Luke, Zachary, Emily, Sophie, James, Hamish, Maya, Mikaela, Isabella, Emma-Jean, Saul, Mia, Daniel, Alexander and Jonas-Jeremy; and grand-friends to Marshall, Daniel and Michael.

Innumerable thanks go to my husband, Michael John Woods, who maintained the running of our home; for being the 'constant' when things got chaotic; and for the dedication to me and the research - go raibh mile maith agat. To my children Karlos, Marshall and Isabella - thank you all for your unconditional love, support and encouragement. As your mother, I have learnt so much about myself as a person - you are my gems! Karlos, during 2010 you have shown me what can be achieved through the power of inner strength and self-belief - I am so very proud of you $\mathrm{xx}$.

Special thanks to my siblings who have all been fortunate to become parents. My three beautiful, creative sisters - Maricia, Lorina and Georgina for their ongoing friendship, wisdom, laughter, practical and emotional support, particularly during the final write up. Your experiences of motherhood that you share continue to be inspiring. Thanks to Joe, Michael and Paul - who have been, and continue to be, supportive and patient brothers but more importantly, caring and present fathers. Thank you to the partners of all my siblings Elisabeth, Paul, Margaret, Nigel, and Simon.

Thanks to my friends who provided so much encouragement and did not mind 'waiting in the wings' in particular Suzanne Millar and Marysia Lobb; and to Louise Greig who introduced me to the wonderful world of research. To 'awesome' Nicola Grace, whose friendship has been, and continues to be, invaluable. Big thanks to my family-in-law, Margaret, John, Peter 
and Cecelia, in particular to Johanna and Jonathan for attending several feis' on my behalf; and for doing the school pick up and drop off run for so long.

To my tolerant and very talented supervisor, Dr Teresia Teaiwa (MOGYM), I thank you for your $100 \%$ support and encouragement; and for nurturing my ideas that enabled me to translate them into words! I am so grateful to Dr Jenny Neale, Dr Lanuola Asiasiga and (soon to be Dr) Fuafiva Fa'alau who never stopped believing in me or my work! I am totally indebted to my wonderful sister-in-law/chief editor, Elisabeth Churchward, who read many drafts of this thesis, sent me great suggestions and insights; and was always available via email - your patience is admirable, thank you. Huge thanks to my mentors Lanuola and Sue Buckley, who sent me interesting articles; listened intently and patiently to my regular "epiphanies" and for the hours of discussions we interpreted as "theory building"!

To all the staff of Health Services Research Centre, Victoria University of Wellington, past and present, who gave some great advice, technical help and their ear along the way in particular Marie Russell, Kirsten Smiler, Dr Lynne Pere, Aliitasi Tavila, Maggy Hope, Jacob Daube and Zachary Gerring. Thanks to Gerardine Clifford-Lidstone for your rescue.

A very special mention to Associate Professor Jackie Cumming whose absolute support and belief in me has been never-ending throughout my entire academic journey, I thank you. 


\section{TABLE OF CONTENTS}

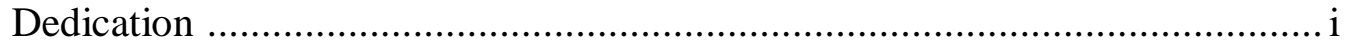

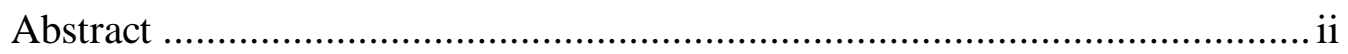

Acknowledgements ..........................................................................

Table of Contents .................................................................................

List of Tables ...................................................................................... ix

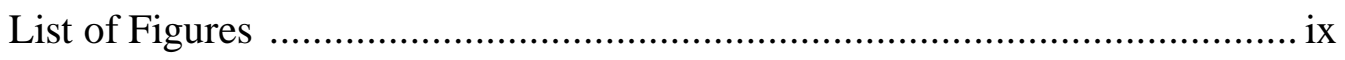

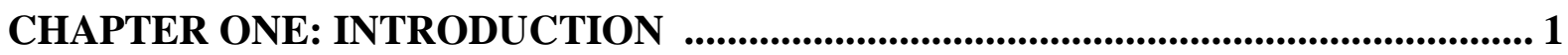

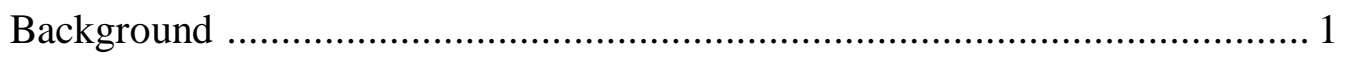

Impetus to carry out this research .............................................................. 1

Sophisticated Mediators ........................................................................... 2

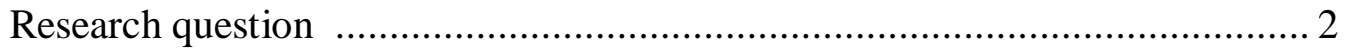

Aims and objectives ........................................................................... 3

New Zealand-born Samoan Women living in New Zealand .......................... 4

Structure of the thesis ................................................................................. 5

CHAPTER TWO: LITERATURE REVIEW ......................................................... 6

Samoan peoples in New Zealand ...................................................... 8

Samoan socio-cultural structure ...................................................... 9

Samoan peoples and their religious affiliation .......................................... 9

Maintenance of the Samoan Language ................................................... 9

The Samoan Self ................................................................................ 9

Health and Wellbeing models for Samoans living in New Zealand ............... 10

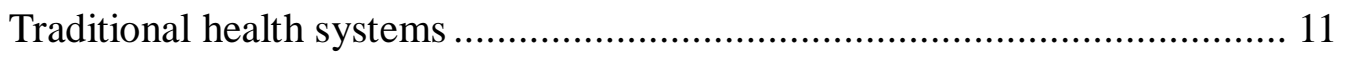

Samoan traditional social position of babies ............................................ 12

Traditional customs and Western practice ................................................. 13

New Zealand-born Samoans ..................................................................... 15

Maternity care services in New Zealand .................................................. 15

Contraception and pregnancy planning/Not planning ........................ 16

$\sim$ Expectations versus experiences ................................................. 17

Antenatal care attendance by mothers ............................................ 18

Motivations to attend antenatal Care ................................................ 19

Antenatal care attendance in New Zealand ......................................... 20

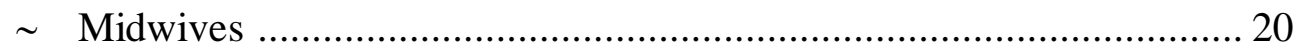

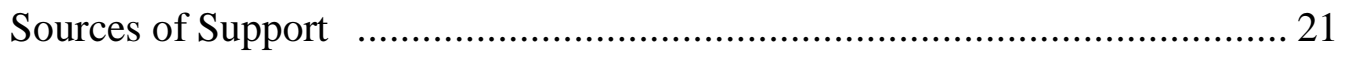

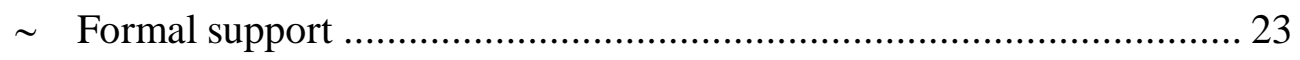

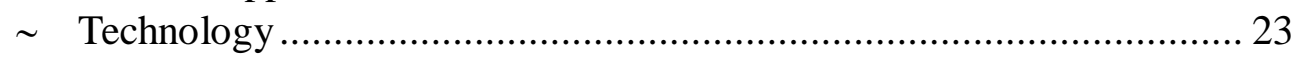

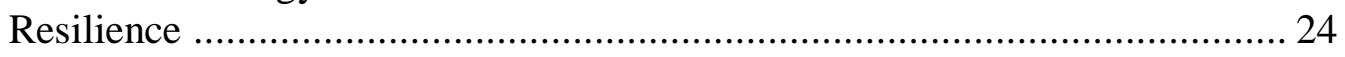

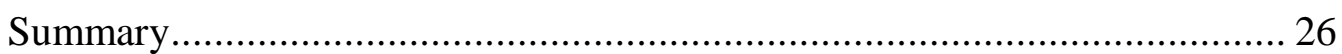




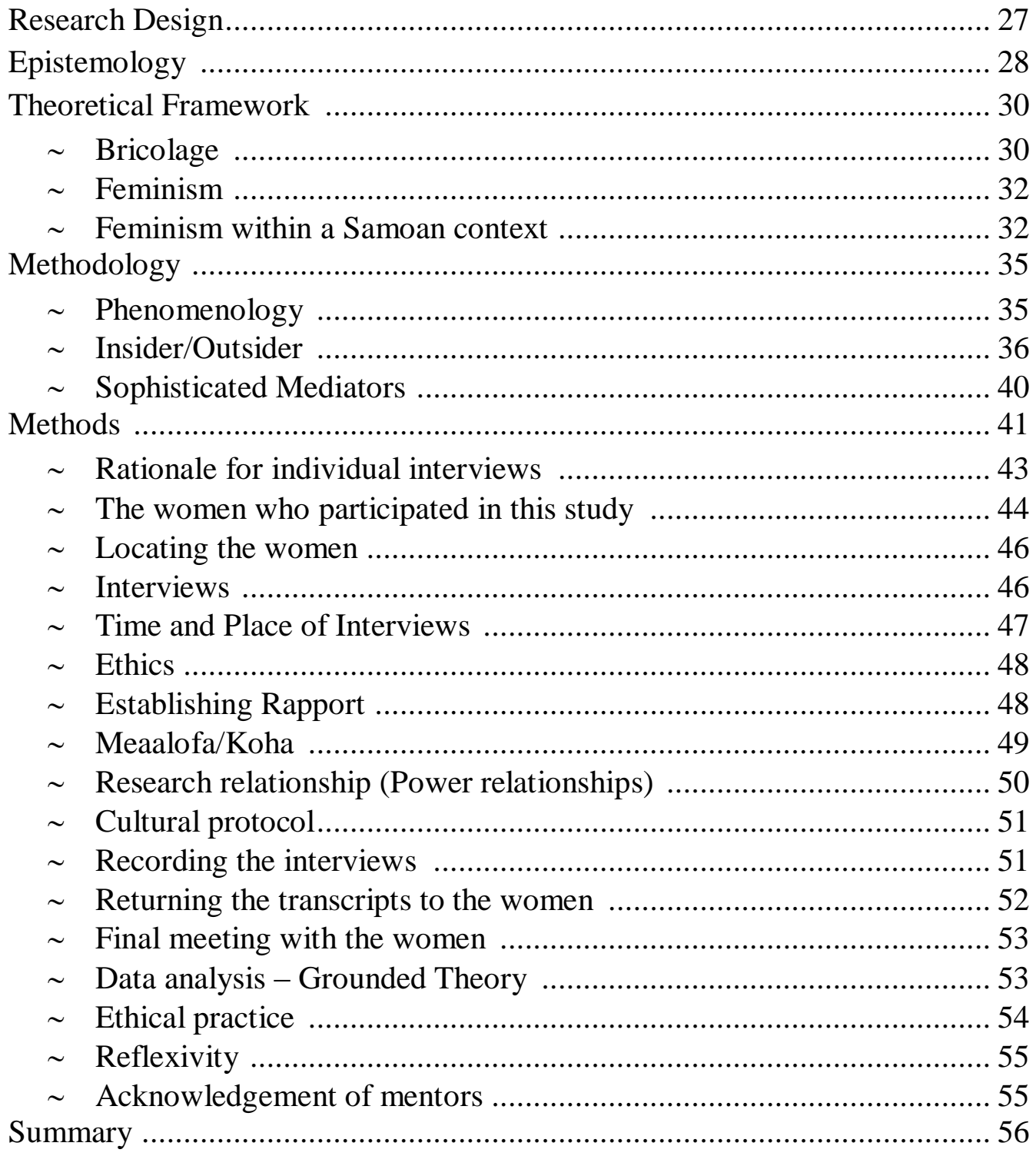

\section{FINDINGS}

CHAPTER FOUR: PREGNANCY

Cultural positions of the women: "I am who I am" ................................... 57

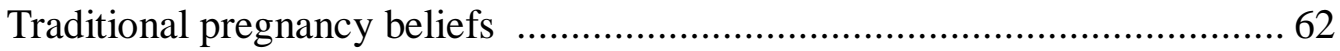

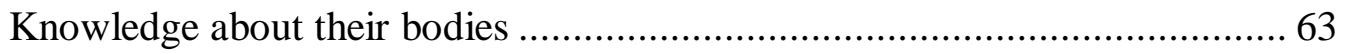

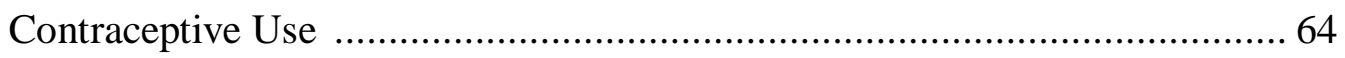

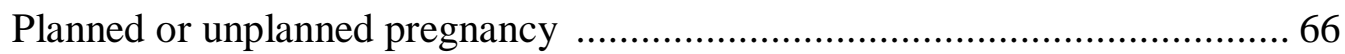

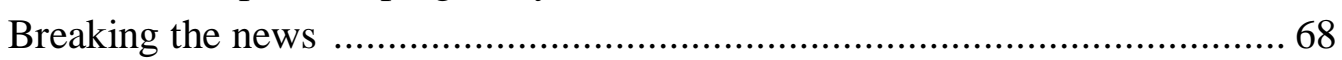

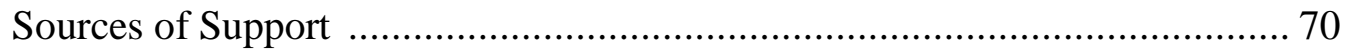

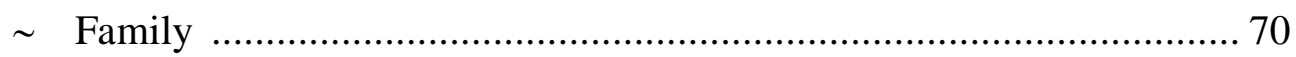




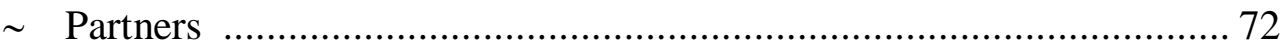

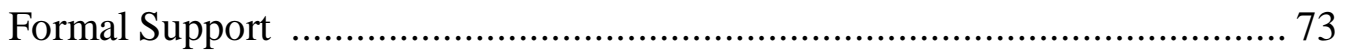

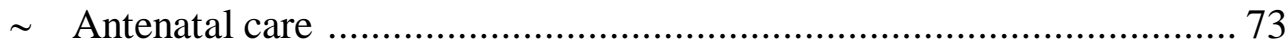

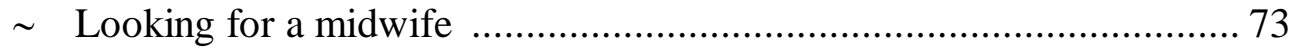

Midwife characteristics ............................................................. 75

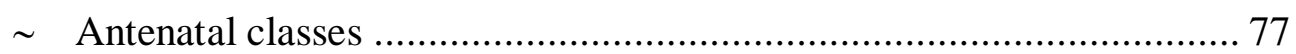

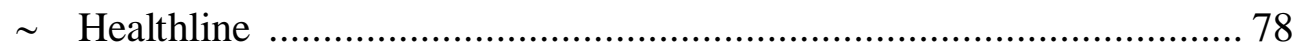

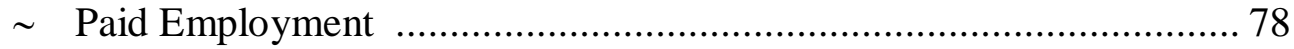

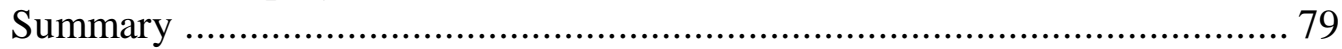

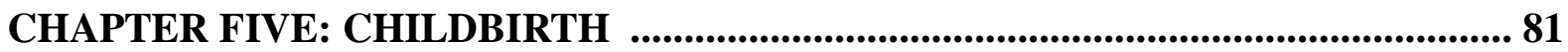

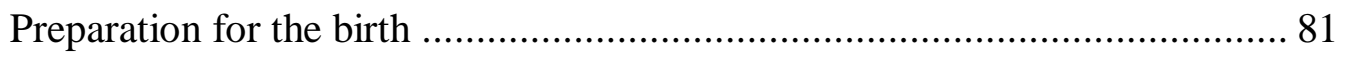

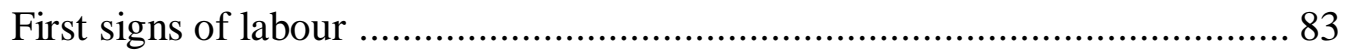

The role of the midwife during labour ................................................... 86

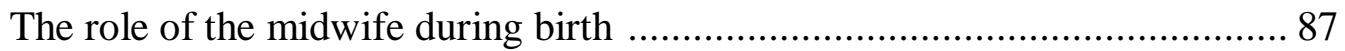

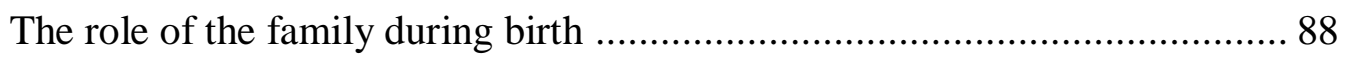

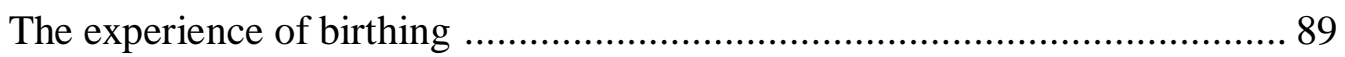

Dealing with a difficult birth ................................................................ 91

Communication with medical staff ........................................................ 93

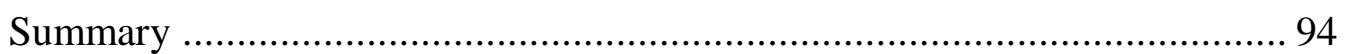

CHAPTER SIX: EARLY MOTHERHOOD ........................................................ 96

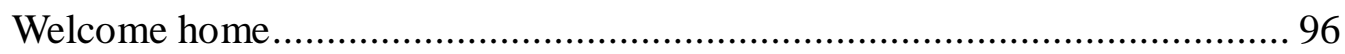

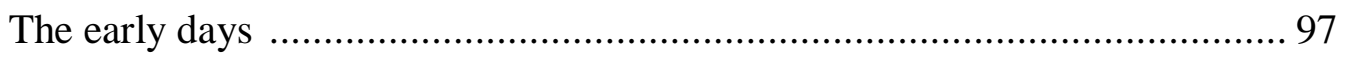

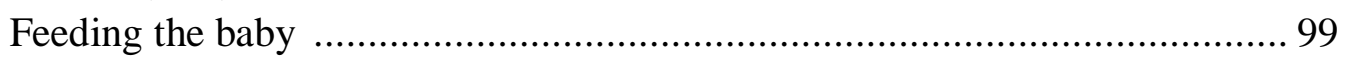

Establishing breastfeeding .................................................................... 100

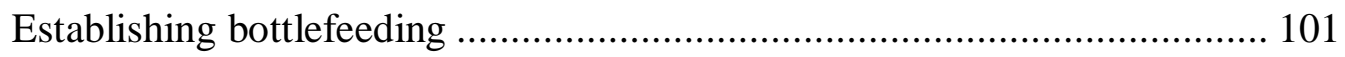

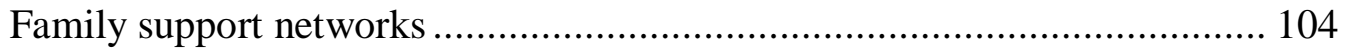

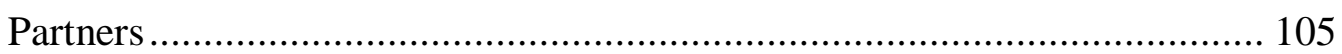

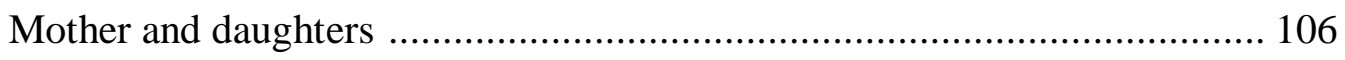

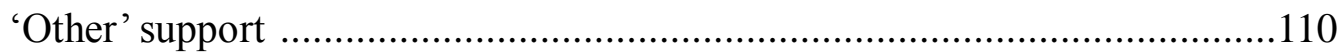

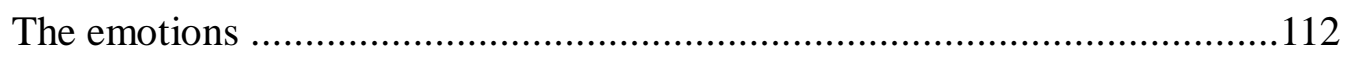

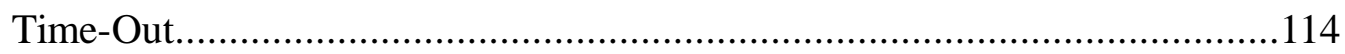

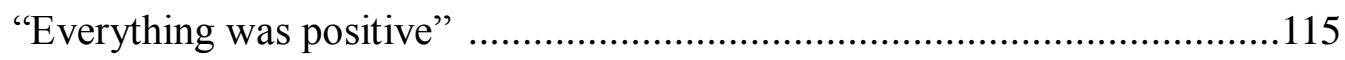

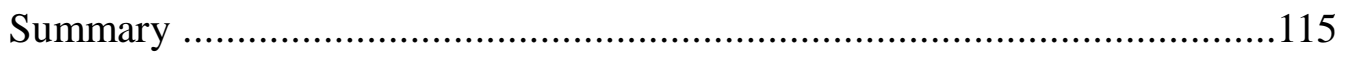

Final words from the women ................................................................117

CHAPTER SEVEN: CONCLUSION

Sophisticated Mediators ...................................................................118

Key factors contributing to health and wellbeing .................................. 121 


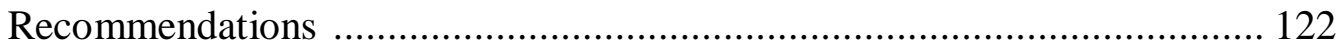

Risk Factors ............................................................................. 123

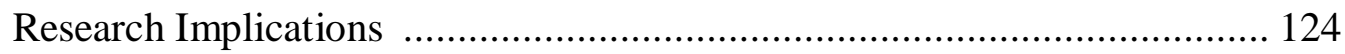

Limitations of the research ............................................................... 124

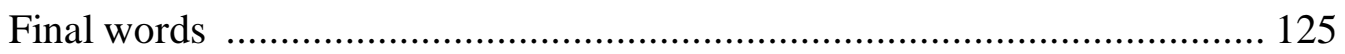

REFERENCES $\quad$........................................................................................................127

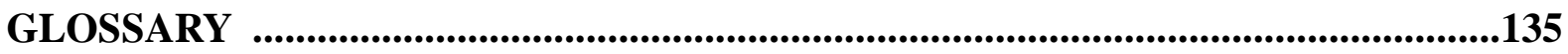

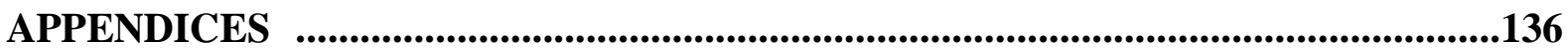

Appendix One:

Participant Information Sheet ........................................................... 136

Appendix Two:

Participant Consent Form/Interview One and Interview Two ................... 137

Appendix Three:

Interview Schedule: Interview One ................................................... 138

\section{Appendix Four:}

Interview Schedule: Interview Two ….................................................. 139 


\section{List of Tables}

Table 1: Information about the women - Antenatal period......................................... 45

Table 2: Information about the women - Postnatal period ....................................... 45

\section{List of Figures}

Figure 1: Fonofale Model 


\section{CHAPTER ONE: INTRODUCTION}

\section{Background}

This thesis is concerned with Samoan women's transition to motherhood - conception, pregnancy, labour, delivery and the early days of motherhood. Statistics show Samoan women living in New Zealand have the lowest prevalence of postnatal depression compared with women in New Zealand and other Pacific Island nations. The rates are not only low by New Zealand standards but also in comparison to international rates.

I first heard about the low prevalence of postnatal depression for Samoan women while I was living in the Republic of Ireland. RTÉ Radio (equivalent to New Zealand's National Radio) was running a news item about postnatal depression which compared the prevalence of postnatal depression in mothers living in Ireland. The segment specifically quoted the findings of the New Zealand study which highlighted the low rates for Samoan women. As a New Zealand-born Samoan mother, this news immediately piqued my curiosity; why was the prevalence of postnatal depression so low in Samoan women? However, it was not until I embarked on an MA thesis that I was provided with an opportunity to explore this question further and, at the very least, hopefully contribute to the body of knowledge on Samoan mothers and their maternal health.

I had two presuppositions as to why this might be occurring: firstly, the possibility that Samoan women did not identify postnatal depression as it was clinically classified (if this were the case then these women were at greater risk as they may not be accessing appropriate support and help). Secondly, if Samoan women did in fact recognise symptoms of postnatal depression they may have kept "silent" for fear of shaming their families (Tupuola, 2000) by being seen as not coping with motherhood or showing weakness. I also theorised the possibility that Samoan mothers' expectations of motherhood were matched by their experiences; therefore they were not disappointed by the outcome. Perhaps also the coping strategies utilised by the women were adequate for their situations. 
The study by Abbott \& Williams (2006) ${ }^{1}$ produced some very interesting statistics on what is very much a public health issue but while the strength of the quantitative data was that it presented a very positive outcome in the case of the Samoan women, it did not provide any rationale as to why this might be happening. As a mother of Samoan descent myself, and one about to embark on a research project, I decided a qualitative component would complement the study and contribute to our understanding of what protects Samoan mothers from postnatal depression during early motherhood.

\section{Sophisticated Mediators}

Sophisticated Mediators is a concept that describes the women in this research, it accurately depicts them in terms of the behaviours they all exhibited i.e. the ability to mediate between their own value and belief system and the socially and culturally constructed systems to which they belong (Samoan and New Zealand). This ability to mediate successfully ensured they sustained or acquired resilience against perceived risk factors. The term Sophisticated Mediators in relation to women and motherhood first appeared in the 1986 article by Patricia Hill Collins, Learning from the Outsider Within: The Sociological Significance of Black Feminist Thought. Hill Collins used the term to describe Black mothers mediating between "the competing offerings of an oppressive dominant culture and a nurturing Black valuestructure" (Hill Collins, 1986, p. S22). However, the redefinition of this concept specifically in relation to this research and the four New Zealand-born Samoan women involved does not ascribe a higher value for the Samoan value-structure over the dominant culture system of New Zealand. Rather, the concept provides the women with a resilient standpoint. The research approach, which allowed the concept of Sophisticated Mediators to emerge, was from a strengths-based position i.e. what can we learn from Samoan mothers who have better emotional outcomes; what key factors promote better health and wellbeing outcomes for these women?

\section{Research question}

The impetus for this current study was the concerns expressed by researchers regarding the limited research conducted to date on understanding why some women even though they are exposed to adverse circumstances including multiple known risk factors (Abbott \& Williams,

\footnotetext{
${ }^{1}$ I acknowledge that on $25^{\text {th }}$ September 2010 (during the final write-up stage of this thesis) it was brought to my attention that the findings from a recent study questioned the validity of the Edinburgh Postnatal Depression Scale (EPDS) for Pacific women (Ryan \& Ekeroma, 2010). The Abbott \& Williams study included the EPDS as a tool to indicate the presence of probable depressive disorder among Pacific Island mothers (2006).
} 
2006), have low rates of postnatal depression symptoms. Samoan women living in New Zealand have the lowest rates of postnatal depression (PND) symptoms both internationally and in comparison with other Pacific groups within New Zealand e.g. Tongan and Niuean. Prevalence rates for Samoan women were $7.6 \%$ in comparison to Tongan women at $30.9 \%$. While there are differences in cultural backgrounds between Pacific Island groups there are many similarities including acculturation and social situation in New Zealand (2006). Some of the findings of the Abbott \& Williams study suggest that maintaining elements of traditional Pacific identity and culture serve as a protective role with respect to postnatal psychological disorder.

Drawing on this, the research question that informed the present study asked: What are the experiences of a group of first-time New Zealand-born Samoan mothers before and after birth? The question around 'experience' was to draw out subjective narratives from women who related their own experience of pregnancy and motherhood. The aim of this project was to explore, in depth, factors that may affect or contribute to the health and wellbeing of Samoan women living in New Zealand during pregnancy and post-birth. The aim was deliberately kept broad in order for the themes to be constructed by data generated by the women themselves. 'Factors' that contribute to the health and wellbeing of Samoan women are classified as positive or negative and this classification will be determined by the women themselves in their descriptions of their own situations.

\section{Objectives}

Family, community and the Church have been identified as important sources of support for Pacific families (Fairbairn-Dunlop \& Makisi, 2003; Tamasese, Peteru, Waldegrave, \& Bush, 2005; Tiatia, 1998; Tupuola, 1998). However, it is uncertain whether the women in the present study viewed them as sources of support or stress or even to what extent they utilised these sources; or, indeed, if they viewed formal support i.e. midwives, antenatal classes, General Practitioners (GPs) as an integral part of the pregnancy and post-birth process.

Therefore, an important objective of this research was for women themselves to define what constituted support systems and key factors that contribute to their health and wellbeing. More specifically, the broad objective was to understand how Samoan women give meaning to their experiences and how they reconstruct their world and themselves in that world 
following childbirth, to identify coping strategies, and; to contrast their perceived expectations against their actual experience.

In essence two main objectives underpin the broad objective of this research, detailed as follows:

\section{Objective 1:}

To undertake a literature review on international and New Zealand research on women and their experiences of pregnancy and post-birth;

\section{Objective 2:}

To conduct narrative interviews with Samoan women:

(i) Identifying a range of support systems utilised by Samoan women during: (a) late pregnancy and; (b) up to 12 months post-birth; to determine the usefulness of these support systems;

(ii) Exploring the impact of pregnancy on the emotional wellbeing of Samoan women;

(iii) Exploring the perceived expectations of Samoan women during the maternity period up to 12 months post birth and whether these expectations were met;

(iv) Identifying coping strategies Samoan women use up to the first 12 months post-birth;

(v) Exploring the impact of motherhood on the emotional wellbeing of New Zealand-born Samoan women;

(vi) Identifying the key factors identified by New Zealand-born Samoan women that have affected their health and wellbeing from the last trimester of the antenatal stage through to the first 12 months postnatal.

\section{Why New Zealand-born Samoan Women living in Wellington?}

Researchers of Samoan heritage have emphasized the unique characteristics of first generation New Zealand-born Samoans (Anae, 2002; Tiatia, 2003; Tupuola, 1998) and in doing so, they have contributed to discussion around the emerging interests of this group which has, in turn, highlighted the importance of conducting further research with this group. This thesis will contribute to the knowledge of New Zealand-born Samoans, particularly in the area of their health and wellbeing. 


\section{Structure of the thesis}

The structure of the thesis followed the natural progression of the research. Chapter One introduces the topic of this research and the impetus behind the choice of this particular topic. It identifies the key research goals, objectives and a rationale behind the research. The next chapter provides a literature review which includes the demographics of Samoan people in New Zealand; a brief overview of traditional Samoan structure including health and wellbeing models for Samoan people living in New Zealand; a review of the studies exploring traditional and Western pregnancy and childbirth practices: the significance of support systems; and the maternity services offered in New Zealand. It introduces the efforts by Samoan people to successfully stake their position within New Zealand society while maintaining their Samoan identity - the first signal of sophisticated mediating. Chapter Three describes the methods and methodology used in this research. The four element model developed by Crotty (1998) provided a framework to conceptualise the foundation for this research. A detailed explanation of the Epistemology, Theoretical Perspectives, Methodology and Methods that informed this research is described. This chapter provides a theoretical premise for successful mediation by integrating traditional and introduced knowledge within an indigenous group. It also features an information table with brief facts about each of the women. Chapter Four discusses the findings and themes that emerged, it introduces the concept of Sophisticated Mediators as first demonstrated by the women in describing the period before and during their pregnancy. Chapter Five covers the period of the childbirth experience. It also describes the women's experiences and the themes that emerged are discussed. The ability to mediate successfully was illustrated during labour and childbirth, a time which, although short, was intense and driven by the need to make quick decisions. Chapter Six provides an insight into the early months of motherhood and the changing dynamics brought about by the introduction of the new baby to the immediate family. Chapter Seven ends this thesis with the key conclusions which emerged: Sophisticated Mediators specifically relates to the four New Zealand-born Samoan women in this study, a concept which clearly posits the women in a position of strength where resilience has been either acquired or maintained and is a solid and positive contribution to the health and wellbeing of these women. Support networks were critical in the overall psychological, physical and emotional sustenance of the women particularly in regards to the extended family, partners, work colleagues, friends and technology. Implications and limitations of this study are also discussed in this final chapter. 


\section{CHAPTER TWO: LITERATURE REVIEW}

In this chapter I discuss the key bodies of literature that help to illuminate a qualitative understanding of Samoan women's experiences of pregnancy, childbirth and early motherhood. I provide an overview of Samoan peoples living in New Zealand. I then present a review of New Zealand and international literature on pregnancy, childbirth and early motherhood. I begin a brief review of the Samoan social structure including perspectives on health and wellbeing. Pacific health and wellbeing models will be highlighted before looking at the wider constructs of pregnancy, childbirth and motherhood including traditional versus Western systems. The concept of support systems will also be discussed.

Pregnancy, childbirth and early motherhood are significant life events for many women, particularly for those experiencing pregnancy for the first time, and they can have lasting meaning for mothers which, in turn, can influence all subsequent reproduction experiences. Pregnancy is also a turning point, a transition (Crouch \& Manderson, 1993; Oakley, 1979), a life crisis; a first baby 'turns a woman into a mother, and mothers' lives are incurably affected by their motherhood; in one way or another the child will be a theme for ever" (Oakley, 1979, p. 24). This life changing event not only affects the woman experiencing the physical and emotional elements of the transition period to motherhood but also has implications for the wider family and community (Oates, et al., 2004); and, although having a baby is a biological process, childbirth and motherhood is ultimately shaped by culture (Oakley, 1980 as cited in M. Stewart \& Hunt, 2004, p. 41). Positive pregnancy and childbirth experiences are found to be important factors in determining the mental and physical wellbeing of women post-birth. These experiences can influence, and be influenced by, the relationship a woman has with her partner, family and herself i.e. her level of self-esteem and can contribute to parenting in a successful way (Dwyer, 2009; Forster, et al., 2008; Lia-Hoagberg, et al., 1990; Melender, 2002; Rice \& Naksook, 1999; Torkan, Parsay, Lamyian, Kazemnejad, \& Montazeri, 2009).

Postpartum or postnatal depression is a significant global public health issue which affects approximately $13 \%$ of women within a year of childbirth (D. E. Stewart, Robertson, Dennis, Grace, \& Wallington, 2003). The impact is far reaching and not only detrimental to the woman's own physical and emotional health and wellbeing but that of her relationship with 
the child, her family, community and wider society (Logsdon, Wisner, \& Pinto-Foltz, 2006; McMahon, Barnett, Kowalenko, \& Tennant, 2006).

Well known risk factors for postnatal depression include: a prior history of depression, depression during pregnancy, marital/partnership relationship problems, single motherhood, lack of support from partner or significant others and recent stressful life events, including bereavement, housing problems and unemployment (Abbott \& Williams, 2006; Crouch \& Manderson, 1993; Robertson, Celasun, \& Stewart, 2003; M. Stewart \& Hunt, 2004). However, risk factors identified in Western populations may not apply to Pacific women and the protective factors for Pacific women may include traditional family and cultural supports, affordable childcare and antenatal education (Abbott \& Williams, 2006). Furthermore, the findings suggest that preservation of aspects of traditional Pacific identity and culture serve a protective role with respect to postpartum psychological disorder.

There is little research on women from minority ethnic groups regarding their experiences of pregnancy, childbirth and early motherhood. Even fewer studies have used qualitative methodology to describe the personal rather than the medical experience of pregnancy for ethnic minority groups in New Zealand. Factors that contribute to their health and wellbeing during this period are also missing from the literature.

This study is intended to fill a gap in social science research by investigating factors that contribute to the health and wellbeing during pregnancy, childbirth and early motherhood of New Zealand-born Samoan women and to contribute to the understanding of why Samoans have low prevalence of postnatal depression when exposed to adverse circumstances. It is envisaged the study may assist in identifying protective factors which would help in the development of prevention programmes for other high-risk groups, not just other Pacific Island groups.

The findings of the current study will be of considerable value as it will highlight reasons why Samoan women in New Zealand show more resilience to postnatal depression than other Pacific Island populations. Factors that contribute to the health and wellbeing of Samoan women may be protective and this will be beneficial in assisting the development of appropriate prevention programmes for Pacific Island and other high-risk groups. 
The literature around pregnancy, childbirth and motherhood has a definitive focus on the health of the infant rather than the health and wellbeing of the mother. Literature specifically around the health and wellbeing of the mother, particularly Samoan first-time mothers, is scarce. Since this study is concerned with Samoan women, it is fitting to begin with a background of Samoan Peoples living in New Zealand.

\section{Samoan Peoples in New Zealand}

There is limited international research investigating the experiences of pregnancy, birth and post-birth of minority groups. This is true, even more so, within a New Zealand context. Samoan women are an ethnic minority group within New Zealand. At the 2006 census (Statistics New Zealand, 2006), the total population for New Zealand was just over 4,000,000 people. Of those, almost 266,000 people were of Pacific ethnicity, an increase of $14.7 \%$ from the 2001 Census $(232,000)$. Pacific peoples make up over 7\% of New Zealand's total population, of which 59\% are New Zealand-born (Statistics New Zealand, 2008a, p. 493).

The Pacific population in New Zealand incorporates at least 13 distinct languages and cultural groups and includes people born in the Pacific Islands and in New Zealand. The many Pacific ethnicities are represented primarily by Samoan, Cook Islands, Tongan, Niuean, Fijian and Tokelauan groups, with smaller numbers from Tuvalu, Kiribati, Papua New Guinea, Vanuatu, the Solomon Islands and the small island states of Micronesia (Statistics New Zealand, 2008b).

Samoan people were the largest Pacific ethnic group, making up just under half (49\%) of the total Pacific population in New Zealand, and of that $49 \%$ over half $(60 \%)$ were born in New Zealand (Statistics New Zealand, 2008c). The majority of all Samoans live in the North Island: Auckland region (67\%) and Wellington region (16\%) (Statistics New Zealand, 2008c). Statistics show that $38 \%$ of all Samoan women in New Zealand over the age of 15 did not have any children. The statistics for other Pacific women and non-Pacific populations were $36 \%$ and $31 \%$ respectively (Statistics New Zealand, 2008c). The percentage of Samoan women who had four or more children was 23\%; the percentage for the total New Zealand population was 14\% (Statistics New Zealand, 2008c). When comparing New Zealand-born Samoan women with overseas born, a higher proportion of those born outside New Zealand had more children; those born in New Zealand were more likely to have fewer or no children (Statistics New Zealand, 2008c). 


\section{Samoan socio-cultural structure}

The social structure of Samoan society, fa'aSamoa, is held together and actively understood both at home (in Samoa) and in the diasporas through a variety of unwritten yet understood cultural conventions (Ngan-Woo, 1985). The governing system of fa'aSamoa involves the social system of rank and conformity that underpin the family and village life. The three principles that formalise the system are based around ava (respect), fa'aalaolo (reverence) and alofa (love, compassion and concern) and are the basis of spiritual and cultural living, the prime motivational force being to safeguard the family status (Fairbairn-Dunlop, 1998; NganWoo, 1985). Religious affiliation, language and the self in relation to others are elements that are critical in maintaining the cultural essence of the Samoan culture.

\section{Samoan Peoples and their Religious affiliation}

Religion plays a major role in the lives of Samoan people and this is reflected in the statistics. At the last census, 2006, 86\% of the total Samoan population in New Zealand declared affiliation to at least one religious denomination; in comparison the total Pacific and New Zealand populations with religious affiliation were $83 \%$ and $61 \%$ respectively (Statistics New Zealand, 2008c).

\section{Maintenance of the Samoan language}

In 2001, the proportion of the New Zealand-born Samoan population able to speak Samoan was unchanged from 1996 i.e. 48\% were fluent in Samoan. Among the New Zealand-born population, the 20-24 year old age group was the most likely to be able to speak Samoan i.e. 54\% in 2001 (Statistics New Zealand, 2008c). The importance of maintaining language as a link to cultural heritage has been documented as protecting against postnatal depression (Abbott \& Williams, 2006).

\section{The Samoan Self}

It is well documented that the family is the central part of Samoan culture. Describing the concept of "self" within a Samoan context is to view the person as a relational being rather than an individual entity and this highlights that the "Samoan person does not exist as an individual" but rather "in relationship with each other" (Airini, et al., 2010; Tamasese, et al., 2005 , pp. 29, 83). In essence, a person always exists in relationships; therefore, the maternal period of a women's life is almost always within a family situation and viewed in this way. 


\section{Health and Wellbeing models for Samoans living in New Zealand}

At this stage it is important to acknowledge some of the currently available models of health and wellbeing for Pacific peoples in New Zealand. Whilst these models will not be utilised as part of the analytical process for this particular thesis, it is acknowledged that these metaphorical and theoretical concepts are important for the future development of Pacific peoples.

While I acknowledge the Tongan Kakala model developed by Konai Helu Thaman and the Cook Island Tivaevae model by Teremoana Maua-Hodge (Kingi-Uluave \& Olo-Whaanga, 2010), I will mainly detail the Samoan models of health which are based on traditional Samoan values and beliefs and holistic views of health and wellbeing. One such model is the Soifua Maloloina which is translated as meaning "life wellness" (Lui, 2007). The cycle of this model is important as it connects the Tagata (People), Atua (God), Laufanua (Land or Environment) together (Ermine, Sinclair, Jeffery, \& Indigenous Peoples' Health Research Centre, 2004). A person who is in a state of wellness is able to meet his or her responsibilities and obligations to self, family, community, and God. Samoans therefore view health in a broad and holistic sense (Lui \& Schwenke, 2003).

The "Fonofale" 2 " model, holistic in nature, encapsulates the individual in relation to the environment and those around them. The traditional Samoan fale (house) is used as a metaphor (See Figure 1 below).

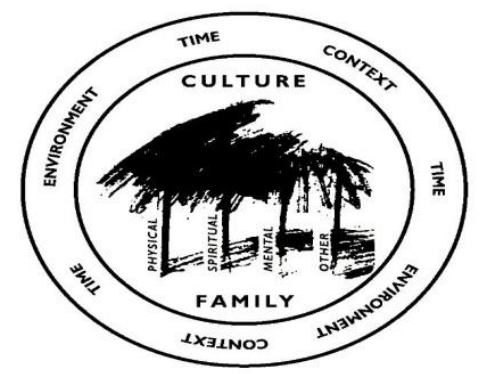

Figure 1 : Fonofale Model http://www.alac.org.nz/DBTextworks/PDF/AODYouthWorkCh4.pdf

The model identifies six dimensions of health. The foundation that the family is built upon represents family, both nuclear and extended family (āiga) and forms the fundamental basis of social organisation. The four pou (posts) represent four further dimensions of health,

\footnotetext{
${ }^{2}$ The Fonofale model was created by Fuimaono Karl Pulotu-Endemann as a Pacific Island model of health for the use in the New Zealand context.
} 
namely: Physical - the wellbeing of the body, which is measured by the absence of illness and pain; Spiritual - the sense of inner wellbeing, encompassing beliefs around Christianity, traditional spirits and nature or a combination of both; Mental - the wellbeing of the mind which involves thinking and emotions as well as the behaviours expressed; Other encompasses variables such as finance, gender, age, education and sexual orientation. The roof symbolises the sixth dimension of culture representing the values, attitudes and beliefs of Pasifika culture. Some families may lean towards a Palagi ${ }^{3}$ orientation where those particular family members practice the Palagi values and beliefs. Other families may live their lives in a continuum that ranges from a traditional Pacific cultural orientation to a Palagi cultural orientation. These can include beliefs in traditional methods of healing as well as Western methods.

The six dimensions of health are encapsulated in a cocoon: the environment addresses the relationships and uniqueness of Pacific people to their physical environment; context relates to the where/how/what and the meaning it has on people; time is relevant to the individual, all of which have either a direct or indirect influence upon one another. The dimensions are interwoven and interdependent, so that altered states of wellness only occur when one or more of the dimensions are out of balance (Kingi-Uluave \& Olo-Whaanga, 2010; PulotuEndemann, et al., 2007). While the Fonofale was developed as a response to the Samoan experience of mental health, other Pacific groups have adapted this model to address the needs of their own Pacific communities (Agnew, et al., 2004; L. Williams, McCreanor, \& Barnes, 2003).

\section{Traditional health systems}

Traditional belief systems around pregnancy and childbearing and Western maternity structures may coexist for women who are part of a minority group, be it Indigenous or ethnic immigrant. These traditional beliefs may conflict with what is considered to be medically acceptable within the dominant society (Oates, et al., 2004).

Historically, in New Zealand some traditional Samoan mothercare methods have been questioned and challenged by Western medical practitioners - in particular, attending a fofo to receive a milimili (massage). In the 1980s the practice was seen to be the cause of the high

\footnotetext{
${ }^{3}$ Definition of Palagi (it is the shortened version Papalagi) meaning: a foreigner (Pratt 1892); European, white man (Milner 1966) (Pratt 1892; Milner 1966, as cited in Asiasiga, 2007, p. 8).
} 
incidence of stillbirth amongst Pacific women in New Zealand due to intracranial haemorrhage. However, these claims were disputed and it was argued by Laing and Mitaera (1994) that there was a lack of sound research evidence to support these claims. Furthermore, it is argued that "the validity given to the claims was due to the dominance of Western medicine over the construction of health knowledge" (Laing and Mitaera 1994, as cited in Abel, Park, Tipene-Leach, Finau, \& Lennan, 2001). The knowledge of traditional health practitioners is holistic in nature rather than individualistic, particularly pertaining to midwives.

Throughout the process, from conception to birth, the main job of the fa' atosaga ${ }^{4}$ (traditional Samoan midwife) is to provide the mother with good advice and utilise massage to assist with a successful birth. Depression and abnormal appetite can easily upset many pregnant women's balance - mentally, physically and spiritually - and the fa'atosaga's regimen of fofo and counselling is therefore a holistic approach. It considers the need for balancing the harmony of the mind and body of the mother paramount, ensuring that these are in balance with the growth of the unborn child (Tamasese Ta'isi Efi, 2009). The indigenous Samoan concept of pregnancy (ma'itaga, ma'ito) is viewed as an illness or a sickness (Kinloch, 1985; Macpherson \& Macpherson, 1990; Tamasese Ta'isi Efi, 2009). It is known as ma'itagata or more commonly known by its shorter name as ma'itaga which essentially is to be ill ( $\left.m a^{\prime} i\right)$ with a new tagata (human being) (Tamasese Ta'isi Efi, 2009, p. 119).

\section{Samoan Traditional social position of babies}

The social positioning of babies is remarkably high in Samoa. Meleisea and Schoeffel point out that in a traditional Samoan village setting, although children are in the lowest status in the aiga, babies and toddlers are often "indulged" up to the age of 2 years or more (Meleisea \& Schoeffel, 1998).

In addition, Samoan babies of single mothers are afforded the same status within a fa' aSamoa social structure as those of married mothers. In her study of the experience of female-headed households in Samoa, Stewart-Withers found that the practice of fa'aSamoa supports the welfare and wellbeing of female-headed households, including children born of those

\footnotetext{
${ }^{4} \mathrm{Fa}$ 'atosaga is a person who is regarded as a specialist (midwife) and a professional by herself and her community but is yet to complete a Department of Health midwife training course. TBA (traditional birth attendants) is the term used to refer to midwives and other women who have attended the Department of Health training course (Barclay, Aiavao, Fenwick, \& Papua, 2005).
} 
households (Stewart-Withers, 2007, p. 283). The importance of the family and the practical aspect of fa'aSamoa ensure the female-headed households do not remain victims of their circumstances and thus are not isolated, shamed or discriminated against. The following quote from a participant referring to an unmarried pregnant woman illustrates this point:

We forgive her, even if she doesn't have a husband. When the baby is born everybody will look after her and the baby. This is what fa'aSamoa is (StewartWithers, 2007).

This is further elaborated upon by Ngan-Woo when he explains that under fa'aSamoa no child is without a aiga (family) regardless of the situation between the mother and father (i.e. if they are unmarried or separated). The ritual fa'ailoga tama must be performed to achieve a reconciliation of both families; this ensures the child's a aiga has recognised that the child is one of its own members. After the ritual, the baby is then culturally legitimised and will not be known as an "illegitimate" child; more importantly, all of the support systems of the aiga are then available to the child with no moral judgement made about either parent (Ngan-Woo, 1985). For Samoan women living in New Zealand, they may take on the principle of this custom rather than have the fa'ailoga tama ritual performed.

A later study exploring the parenting experiences of Pacific Island single mothers in New Zealand found that within the New Zealand social context, the maintenance and strengthening of their cultural connections - particularly family - enhanced the young mothers' experiences (Stewart-Withers, Scheyvens, \& Fairbairn-Dunlop, 2010). Abel et al confirm that strong family support for pregnant women and new parents was assumed and readily available, as the children's wellbeing was the responsibility of the extended family (also see Ngan-Woo, 1985). However, others have also pointed out that in the case of unmarried pregnant women, family support initially could be equivocal because of the stigma to the family of an unplanned pregnancy confirming the woman is no longer a virgin, but that was usually only temporary (Abel, et al., 2001; Tupuola, 2000). The overriding cultural aspect may also act as a protective factor.

\section{Traditional custom and Western practice}

For some women, overlooking traditional customs in favour of Western practice can be liberating. Rice and Naksook (1999) interviewed 30 Thai women living in Australia to identify their perceptions and experiences of pregnancy care, labour and birth. A distinction 
was drawn between traditional and Western practices, especially for the women who had previously given birth in Thailand. Within Thai tradition, screaming and crying is discouraged as the ability to keep silent is perceived to indicate the maturity of the woman. Crying out, groaning, and moaning are considered childish and inappropriate responses that imply a future inability to care for the newborn (York, Bhuttarowas, \& Brown, 1999). However, many of the participants in the Rice \& Naksook study commented that being "allowed" to scream and cry during birth in Australia (Rice \& Naksook, 1999) provided a sense of relief from the pain and gave them the option of choosing to be silent or vocal during childbirth. Thus, they chose to opt out and not follow their traditional Thai childbirth ritual. This autonomy allowed the women to freely express their emotions, giving them more control over their birth event and thus increasing the positive experience of childbirth.

Not all migrant women, however, forgo traditional beliefs. In her study examining the ways in which migrant Samoan women gave birth in Wellington, New Zealand in the mid-1980's, Donnelly states that "Samoan women come to New Zealand already aware of an obstetric or medical way of doing birth as well as a distinctly Samoan way" which is less dominated by the biomedical paradigm (Donnelly, 1992, p. 55). In addition, in terms of diagnosing and treating general health and illness, Samoans in Samoa draw from both Samoan and Western knowledge systems (Macpherson and Macpherson as cited in Donnelly, 1992; Norris, Fa'alau, Va'ai, Churchward, \& Arroll, 2009). This is supported by other studies that have shown that migrant Samoans living in New Zealand utilise both Western medical and Samoan healing systems in the treatment of illness (Kinloch 1980 as cited in Donnelly, 1992).

However, others suggest that while many New Zealand-raised Pacific parents see the value in traditional beliefs and values, many find it difficult trying to marry two very different belief systems (Abel, et al., 2001; Tiatia, 2003). Yet, having the ability to access and utilise both Western and traditional Samoan systems may be a source of empowerment for pregnant women, particularly in New Zealand where the dominant health system is perceived to be the appropriate method. However, the confidence of the mother to access both systems may rely on many factors, the least of which may be the appropriateness or the social acceptability of whichever she might choose. 


\section{New Zealand-born Samoans}

The duality of roles played out by New Zealand-born Samoans attempting to navigate what some essentially see as two opposing world views, is well documented (Tiatia, 1998; Tupuola, 1998). The "struggle" to keep the culture of their parents alive while contributing to an emerging culture has been to bridge the gap or merge between the two (Anae, 2002). In addressing the New Zealand-born identity, Tupuola clearly articulates that not all young New Zealand-born Samoans experience a "cultural dilemma or identity crisis", just as not all of them see themselves as a "bridge" between the two cultures (Tupuola, 1998, p. 62). In her dissertation examining Pacific women's stories of becoming nurses in New Zealand, Margaret Southwick describes the women in her story as "mediating between discontinuous worlds". It highlights the women's vulnerability when trying to negotiate between the two worlds; some, eventually, are forced to choose one world over the other in order to "fit in" (Southwick, 2001, p. 80). This mediation created much tension as the women struggled to essentially fit both worlds into their realm. However, Southwick refers to one of the women being successful in her mediation:

She has found a way of mediating the tensions between her two worlds by simply acknowledging that they are different worlds and is very clear about not getting the roles muddled up. She makes no attempt to integrate them. They are separate and different and she moves with confidence between them. It is very likely that this clarity is what enables her to stand her ground. (Southwick, 2001, p. 98)

Southwick makes a salient point in reiterating the two worlds are "different", however, it begs the question of whether "moving between" means letting go of one's culture to fit in another and whether it is really "success" or more defined as conforming to each of the separate cultures. Furthermore, "discontinuous" or "opposing" worldviews may or may not really be discontinuous or opposing which is heavily dependent on the views and cultural backgrounds of the women.

\section{Maternity Care Services in New Zealand}

Maternity services in New Zealand are designed to offer women and their families' choice in who cares for them and continuity of that care. Care in pregnancy and childbirth is free for New Zealand citizens and permanent residents, or for their partners or spouses, except for care provided by private obstetricians and at private hospitals. This care covers the diagnosis of pregnancy, antenatal care, childbirth, postnatal care and miscarriage. Women who are not 
eligible for publicly funded health services may be charged for antenatal, labour, birth and postnatal services provided to them. Also, babies born from 1 January 2006 will only be eligible for New Zealand citizenship if at least one of their parents is a New Zealand citizen or permanent resident. If you are not eligible for publicly funded maternity services and your baby is not eligible to be a New Zealand citizen, you will also pay any costs for your baby (New Zealand Ministry of Health, 2010c).

\section{Contraception and Pregnancy Planning/Not Planning}

The complexity of the intent to become pregnant cannot be underestimated. A planned pregnancy, an unplanned pregnancy, or an unconscious decision to become pregnant is complex and complicated. Women possessing several characteristics of an unplanned and unconscious decision to become pregnant indicate a lack of a "firm commitment" (StevensSimon, Beach, \& Klerman, 2001). Other studies have a third category of women who were "okay either way" about becoming pregnant or not becoming pregnant (McQuillan, Greil, \& Shreffler, 2010). A study exploring reasons for the high rate of unintended pregnancy among low income women in Georgia (USA) found that women who strongly desire to avoid pregnancy select an effective contraceptive method and use it consistently, whereas women who are undecided about pregnancy will consider the cost of contraception as higher than the cost of pregnancy (Lifflander, Gaydos, \& Hogue, 2006).

A New Zealand study exploring pregnancy planning found the rate of unplanned pregnancies among Pacific mothers in New Zealand to be $60 \%$ and, of those mothers, almost three quarters of the women were not using contraception when they conceived (Paterson, Cowley, Percival, \& Williams, 2004). Some of the reasons cited by the women as to why they were not using contraception included: did not like using contraception, decided to take a chance and did not want to risk the associated weight gain (2004). In her research with Samoan women and pregnancy, Donnelly (1992) found that first time Samoan mothers not using contraception at the time they conceived were overrepresented in her sample. Reasons for low contraceptive use among Samoan women may be due to: strong religious and family values, the high social and cultural value placed on children, a lack of discussion about sexuality and the fear and embarrassment of potentially being seen at family planning services. These examples are seen as real barriers to accessing contraceptive services for many Pasifika people (Asiasiga, 1994; Collins, 2005; Fa'asalele Tanuvasa, 1999). 


\section{Expectations versus experiences}

It is well documented that factors affecting the health and wellbeing of women during this significant life event include expectations and experiences of pregnancy, childbirth and early motherhood. These include maternity care, childbirth, postnatal care and support systems that include families (for example Abbott \& Williams, 2006; Cox, Holden, \& Sagovsky, 1987; Oates, et al., 2004; Robertson, Grace, Wallington, \& Stewart, 2004; Watson, Hodson, Johnson, \& Kemp, 2002). A systematic review using several databases to obtain studies relating to the experience and expectations of pain and its relief during childbirth found that if women were well prepared during pregnancy they were more likely to have "realistic expectations of pain" which would lead to a more positive experience. The authors also found that if there was a mismatch between the level of pain expected and that actually experienced then it was more likely to cause a less favourable experience of the labour (Lally, Murtagh, Macphail, \& Thomson, 2008).

In a New Zealand study examining the relationship between birth experience and depression 10 days post-birth, participants were involved in self-reporting via a questionnaire in the last trimester of pregnancy and again each day during their hospital stay - on average 7 days. The authors found that the mismatch between the expectation of birth, birth experience and whether the birth mismatched the expectation was not a predictor for postpartum blues. They also found that the predictor for depression was the anxiety and fear of birth itself rather than the match of experience and expectation and that the level of depression during pregnancy was the best indicator for depression post-birth (Knight \& Thirkettle, 1987). The limitations of this study were that all the women were married, most held a post-college degree, all were recruited from an antenatal class from one geographical area and all the questionnaires were completed within a hospital setting.

However, a meta-synthesis exploring the development of postpartum depression found that there was a disparity between women's expectations and the reality of motherhood in several areas including: labour and delivery, life with their infants, self as a mother, relationship with partners, support from their family and friends. These, according to Robertson, Celasun and Stewart (2003) were factors that contribute to postpartum depression (Robertson, et al., 2003, p. 52). They alluded to the term "dangerous myths" in which becoming a mother equates with fulfilment and happiness. In addition, the often unrealistic expectations were shattered by 
their experiences of not attaining the "perfect" mother status (Nicolson, 1990 as cited in Robertson, et al., 2003).

A sense of control, whether perceived or actual, has been found to be an important characteristic of women's satisfaction with the childbirth experience as found by Gibbens and Thomson (2001). In their study of eight pregnant women expecting their first baby, they found that one factor which helps women feel confident about childbirth was being in control of the situation. Although the women's experiences of labour, pain and delivery were described as different from what they expected, all the women expressed positive feelings (Gibbins \& Thomson, 2001; World Health Organisation, 2009).

Another study exploring the impact of maternity care staff on women's experiences and feelings associated with the childbirth process found that, while their physical aspects of care were sufficient, the psychological dimensions were seen as insignificant. Similar to other studies, the perceived lack of control, few choices, and little influence over decision making were all issues raised by the women. Along with these issues, the "(negative) attitudes and behaviour of some staff" meant women would often underreport negative experiences. This was associated with "a range of feelings including disappointment, anger, inferiority, inadequacy and bullying" (Baker, Choi, Henshaw, \& Tree, 2005, p. 332).

\section{Antenatal care attendance by mothers}

While many profess that adequate antenatal care during pregnancy contributes to a more positive wellbeing for the mother (Lavender, Downe, Finnlayson, \& Walsh, 2007; Low, et al., 2005; Robertson, et al., 2004), there are others who believe that antenatal care represents one way of "controlling women" and the benefits are not universal and may be appropriate to selected groups of women in selected localities (Oakley, 1984). This form of "control" according to Oakley is by not acknowledging women's own experiences of pregnancy, both culturally and traditionally; by replacing her own experience with information and knowledge using techniques and medical interventions by the medical expert in midwifery (Oakley, 1984).

Barriers to seeking antenatal care and whether it is considered adequate include a variety of inter-related socio-economic, educational, cultural and personal factors including: structural or financial difficulties, education, other family commitments or poverty (as cited in Rice \& 
Naksook, 1999), cost of transport, late recognition or denial of the existence of pregnancy, reliance on traditional/tribal (herbal, spiritual) pregnancy beliefs, lack of perceived benefits and influence of partner (Lavender, et al., 2007). Lia-Hoagberg et al. (1990) found that being poor often led people to feel they had few options and little control over basic life decisions: "In the process of putting food on the table, caring for other children, coping with everyday stresses of being poor, and dealing with the worries engendered by the pregnancy, significant numbers of women found it difficult to give high priority to prenatal care" (Lia-Hoagberg, et al., 1990, p. 493).

Geographical proximity or otherwise was also a factor as to whether or not some women attended antenatal care services in their communities. For some women, the absence of a clinic or limited availability of classes is a barrier for attending antenatal care, particularly in remote communities as was the case for Indigenous mothers from the Northern Territory of Australia (Watson, et al., 2002). Language barriers and the lack of culturally appropriate services can also hinder women seeking antenatal care.

\section{Motivations to attend antenatal care}

Some have argued it is not the barriers to seeking antenatal care that should be the focus; rather, focus should be on what motivates women to seek antenatal care. Motivators may include reasons such as the safety of the baby, good relationships with medical professionals, personal autonomy, encouragement and support from others to seek care (Lia-Hoagberg, et al., 1990; Rice \& Naksook, 1999). Rice \& Naksook (1999) found that Thai mothers living in Australia conformed to antenatal care and regularly attended appointments even though they had limited English and had to depend on their partners to take them to the appointments. They saw antenatal care as an important aspect of their pregnancy and sought care as soon as they suspected they were pregnant. However, they were more concerned about the wellbeing of their babies rather than their own health (Rice \& Naksook, 1999). Encouragement from others such as partners, health professionals, and the wider family also contribute to women seeking antenatal care.

With this in mind, many researchers support the contention that attending antenatal care and receiving good social support during and after pregnancy contributes to the health and wellbeing of women during childbirth and postnatal experience. It may even act as a protective factor against developing depression after childbirth (Goodman, Mackey, \& 
Tavakoli, 2004; Rice \& Naksook, 1999; Righetti-Veltema, Conne-Perreard, Bousquet, \& Manzano, 1998; Robertson, et al., 2004). However, while women may see antenatal care as important and are just as likely to be concerned about the wellbeing of their child, it does not mean they will attend antenatal care regularly or early (Lia-Hoagberg, et al., 1990).

\section{Antenatal Care Attendance in New Zealand}

Pacific women living in New Zealand have very low rates of antenatal attendance, despite the fact that maternity care in New Zealand is free to women who are residents and citizens (New Zealand Ministry of Health, 2008). Despite this, studies show that Pacific women had better pregnancy outcomes, with lower postnatal depression rates for Samoan women, lower preterm and small gestational age rates when compared with non Pacific women (Abbott \& Williams, 2006; Ekeroma, Craig, Stewart, Mantell, \& Mitchell, 2004).

A study examining factors affecting late initiation and attendance to antenatal care by mothers of Pacific infants living in New Zealand found women who had experienced giving birth before were less likely to seek antenatal care early in the pregnancy, suggesting that these women feel their experience warrants them not to seek antenatal care early as they know what to expect (Low, et al., 2005). For first-time mothers factors include lack of knowledge or experience with pregnancy and childbirth; ambivalence over the pregnancy and its outcome; language barriers, particularly for Pacific-born, and low cultural alignment to the New Zealand way of life and customs meant these women were also more likely to initiate their care late (Low, et al., 2005).

\section{Midwives}

Other than family members, midwives are one of the most significant other members of women's support network during the maternal period. Registration with a midwife usually commences during pregnancy, continuing to the first six weeks post-birth; however, continuity of care with the same midwife is not always a given. And yet, despite the majority of women registering with a Lead Maternity Carer (LMC) there is a shortage of midwives to provide LMC care. The latest Maternity Services Consumer Satisfaction Survey in 2007 found that close to one in five women (19\%) found it difficult to find an LMC to provide care for them. Some of the reasons women gave for the difficulty in finding an LMC were that midwives were too busy or that there was a shortage of midwives in their area. This is similar to other research in which many women reported having to make numerous phone calls in the 
search for a midwife. It was not uncommon for women to report making 10-15 calls before giving up and being told to go through the hospital system (i.e. use hospital midwives) instead. Some women had to make compromises (in their choice of LMC or hospital) to secure an LMC (Dwyer, 2009).

Several studies have found that continuity of care by midwives is associated with high levels of maternal satisfaction (Hodnett, 2002). A study evaluating mothers' satisfaction with continuity of care with the same midwives in Australia found that women preferred continuous care and found it fosters the development of supportive relationships between the woman and her midwife (K. Williams, Lago, Lainchbury, \& Eagar, 2009). Some of the advantages of this level of care included the women's feelings of reassurance that the midwives know and respect them, understand their previous experiences and their expectations, adding "it seems naïve to suggest that women would choose to be supported by strangers if there was an alternative" (K. Williams, et al., 2009, p. 7).

Furthermore, the study found that first-time mothers in particular were dissatisfied with their level of care, particularly those relating to feelings of control, participation in decision making and whether they were treated as individuals (K. Williams, et al., 2009).

\section{Sources of Support}

Support is a concept that emerges from much of the research on women and pregnancy; not only does the mother benefit from being well supported but also the family as a whole, particularly the child.

Robertson et al (2004) views support as a multidimensional concept, describing different types of support systems. For example, sources of support can be a spouse, relatives, friends, or associates; types of support include informational support (where advice and guidance is given); instrumental support (practical help in terms of material aid or assistance with tasks), and emotional support (expressions of caring and esteem). Furthermore, studies have found that "perceived social isolation (or lack of social support) during pregnancy was a strong risk factor for depressive symptoms" (Robertson, et al., 2004). 
Mann et al (1999) in their study interviewed African-American nurse-midwives who had experienced pregnancy and had extensive professional experience in the provision of health care services to pregnant African-Americans. The women were asked to define both informal and formal types of support that were helpful during pregnancy and childbirth. The results included material resources (food, transportation, housing, and money) and emotional resources (caring, affirmation, and power); a lack of this support was viewed as a source of stress for the mothers. The women interviewed viewed pregnancy as a normative transition from childhood to adulthood, a transition to maturity, and this was reinforced by prevailing social values (Mann, et al., 1999). A limitation of the study is that the women were also professionals who were more likely to have an intimate understanding of the maternity system; the study also does not draw out any characteristics of coping strategies these women utilised to get them through this transitional period. Nor did it specify whether the lack of material and emotional resources contributed to women not seeking support during pregnancy.

Family, community and the church are considered to be important sources of support however, it is uncertain whether these sources are viewed by the women as a form of support or hindrance (the existence of a social network does not equate to support) (Fairbairn-Dunlop \& Makisi, 2003; Tiatia, 2003; Tupuola, 1998). Therefore, women may be forced to look outside of the family, community and church networks for support (Donnelly, 1992; Tiatia, 2003; Tupuola, 1998). This might be due to inter-generational tension between Island-raised parents, who hold firmly to traditional beliefs and practices, and their New Zealand-raised children, who have begun to challenge the traditional beliefs (Meleisea \& Schoeffel 1998; Tiatia, 1998 as cited in Abel, et al., 2001; Tupuola, 2000).

For many women, particularly immigrant and indigenous, being physically and culturally separated from support systems is a well established risk factor for postnatal depression (Robertson, et al., 2004). A study by Watson, Hodson, Johnson, \& Kemp (2002) exploring maternity experiences of the Aboriginal women of Australia found that indigenous women, particularly in remote areas of the Northern Territory, are encouraged to birth their babies in regional hospitals. The hospitalisation during the birthing experience removes the Indigenous women from their traditional setting, their "special place". This leads to feelings of isolation, disempowerment and disconnects them from receiving care traditionally provided by grandmothers or aunts. Care provided by midwives, who may be non-indigenous with little 
knowledge of the Indigenous culture, can lessen what is expected for these women and further isolate them (Watson, et al., 2002).

\section{Formal support}

The link between well-being and social support and this relation between social support, mental health, and wellbeing has been widely investigated (Oakley, 1992). An operational definition of social support: "Provision of a non-judgemental listening ear, discussing with women their pregnancy needs, giving information when asked to, and carrying out referrals when appropriate to other health and welfare professionals and voluntary and statutory agencies" (Oakley 1994 as cited by Finfgeld-Connett, 2005).

\section{Technology}

Currently, technology including telephone, internet and personal cellphones are now available in most homes and this opens up a new social world, particularly for women at home in the early days of motherhood. While there are many telephone helplines available offering different types of support, there are limited helplines available for women during pregnancy and motherhood i.e. currently there are only two telephone advice services ${ }^{5}$ in New Zealand that would be appropriate to serve women during pregnancy and new motherhood.

In a study looking at telephone-based interventions, trained telephone-intervention nurses contacted participants at least once a week to deliver social support. Participants were able to page the nurses if they needed more support outside of their regular weekly calls. The results found that social support via telephone provides solace and promotes changes in behaviour when individuals are experiencing "powerlessness, isolation, and anxiety" (Finfgeld-Connett, pg 26). In addition, although telephone-based interventions do not accommodate physical (face-to-face) presence:

The study nurses worked diligently to establish psychological, or "mind-to-mind," presence (Easter, 2000, p. 366). This phenomenon is consistent with Oakley's (1994) characterization of social support as the "provision of a nonjudgmental listening ear"

\footnotetext{
${ }^{5}$ Healthline is a national telephone health service provided by the Ministry of Health and delivered through contracted service provider. All calls are answered by registered nurses with telenursing training who assess the caller's health needs and provide information and advice as to the best level of care (New Zealand Ministry of Health, 2010b). Plunket provides free services to New Zealand families for children aged from birth to 5 years old. One such service is PlunketLine, a free telephone advice service available to all families, whānau and caregivers. Calls are answered by a Plunket nurse, who can give advice and information on parenting issues and children's health and wellbeing (Plunket, 2010). Both services are tollfree, 24 hours a day, seven days a week. Healthline also offer an additional service of an interpreter for callers whose English is their second language.
} 
(p. 31). One way this type of therapeutic milieu was established was by liberally offering reassurances that the project nurses were constantly available (FinfgeldConnett, 2005, p. 26).

Access to the internet and personal cellphones (for texting) lends itself to a multitude of social and informational arenas to be explored.

\section{Resilience}

Studies have found that key risk factors are not necessarily the converse of protective factors (Oates, et al., 2004). This study investigating factors contributing to happiness and unhappiness found that following delivery, although tiredness and lack of sleep was a contributor to unhappiness, feeling physically well and having sufficient sleep were not seen as contributors to happiness (Oates, et al., 2004, p. 12 ). Therefore, it cannot be assumed that risk factors are necessarily the opposite of protective factors. Or, put another way, the absence of a risk factor is not necessarily a protective factor (for example perceived high level of support). The known risk factors that contribute to postnatal depression include but are not limited to: clinical history of depression, maternity blues, low-socio economic status, low or no partner support, birth experience, death of someone close, relationship breakdowns, moving home, pregnancy/childbirth complications, breastfeeding difficulties and unplanned pregnancies (Abbott \& Williams, 2006; Milgrom, et al., 2008; D. E. Stewart, et al., 2003; M. Stewart \& Hunt, 2004).

A general definition of resilience is defined as "a dynamic process whereby individuals show adaptive functioning in the face of significant adversity" (Schoon, 2006 as cited in Evans \& Becker, 2009, p. 17). The concept of resilience highlights strengths in being able to cope with adversity. Evans \& Becker (2009) affirm that rather than focusing on risk factors, the concept of resilience highlights people's strengths in coping with adversity and in coping with protective factors that may help to reduce their vulnerability. Protective factors, Evans \& Becker add, may be associated with (a) individual attributes, such as problem-solving skills, high aspirations, faith and religious beliefs, positive peer relationships; (b) family characteristics, such as caring and supportive family relationships, a secure base as the availability of external support or resources (p. 19). Michael Rutter argues that while "positive experiences in themselves do not exert much of a protective effect, they can be helpful if they serve to neutralize some risk factors" (p. 119). 
Rutter, from his earlier studies, found that institution-reared young people who had positive school experiences were more likely to plan in relation to life decisions such as marriage and careers. However, it was unknown why, but it was postulated that success in one arena served to enhance self-esteem and self-efficacy, making it more likely that the individuals concerned would feel more confident that they could handle new challenges and therefore acted accordingly (p. 131). In addition, as in Evans \& Becker (2009), Rutter suggested individual qualities also play a role in positive chain reactions - for example positive temperamental features are likely to elicit warm responses from other people.

In a large international, multi-site, multi-country study of resilience among youth ages 12-23 years, Michael Ungar and his colleagues acknowledged that while there was no "uniform explanation" for what constitutes as resilience, a contextually relevant understanding of resilience emerged from their findings (Ungar, et al., 2007, p. 294), interpreted as seven tensions - (1) Access to material resources includes financial, educational, medical and employment assistance, and/or opportunities, food, clothing, shelter; Relationships includes with significant others, and within one's family and community; Identity includes personal and collective sense of purpose, self-appraisal of strengths and weaknesses, aspirations, beliefs and values including spiritual and religious identification; Power and control includes experiences of caring for one's self and others; the ability to affect change in one's social and physical environment in order to access health resources; Cultural adherence includes adherence to one's local and/or global cultural practices, values and beliefs; Social justice includes experiences related to finding a meaningful role in community and social equality; and Cohesion - balancing one's personal interests with a sense of responsibility to the greater good; feeling a part of something larger than one's self socially and spiritually (Ungar, et al., 2007, p. 295). Pointedly, as Ungar et al remarked, those who experience themselves as resilient and are seen by their communities as resilient are those "that successfully navigate their way through these tensions". Furthermore, in reference to their young participants their study revealed:

Resilient youth find a way to resolve all seven tensions simultaneously according to the strengths and resources available to the youth individually, within their family, community and culture. It is the fit between the solutions youth try, and how well their solutions address the challenges posed by each tension, within the social and political constraints of their community, that contributes to a young person's experience of resilience (Ungar, et al., 2007, p. 294). 
The ecological model proposed by Ungar et al is not constricted within a linear, causality form. The authors acknowledge that while the model has an illusion of "permanence" they accept that "impermanence" more closely approximates reality.

\section{SUMMARY}

A review of the current literature confirms the limited material available on factors related to women who have low prevalence rates of postnatal depression. There is a wealth of information pertaining to the mismatch of the expectations and experiences of the maternal period that affect mothers in the postnatal period and the impact this has on new mothers and their families. While the impact is not always negative, it is significant to understand the factors that contribute to a positive outcome.

The Samoan-specific literature offered a brief insight into the intricacy and paradoxes of Samoan culture i.e. the high status of children versus the hierarchical nature of the Samoan social structure. The traditional ritual to break down the stigma associated with single motherhood co-existed with strong religious affiliations that challenge sex before marriage ideals. The evidence that Samoan women come to New Zealand experienced at mediating between health systems indicates an ability to mediate between social structures within different cultures. The cross-cultural literature from Australia provides an insight into other migrant groups who are also part of a minority cultural group within a dominant culture society. 


\section{CHAPTER THREE: METHODOLOGY}

This chapter is essentially divided into four parts: Epistemology, Theoretical Framework, Methodology and Methods. It begins with the epistemological frameworks that underpin the concept of knowing and an introduction to an indigenous epistemology is complemented by a social constructionist epistemology in order to describe "how we know what we know". A bricolage approach was taken to inform the theoretical framework as the one-size-fits-all model was not appropriate for the type of study or for the group of women involved. The women can be considered bricoleur themselves in that they drew from several sources available to them during their pregnancy and motherhood experiences. Feminism, social constructionist and Pacific studies were drawn together to realise bricolage. Drawing on the epistemological and theoretical frameworks, the Methodology section outlines the research methodology which is best described as feminist phenomenological social constructionist. Furthermore, this section details a discussion of insider/outsider/outsider-within and is articulated as positions that are not fixed or static; highlighted are some of the dynamics of the insider/outsider/outsider-within which shaped relationships between myself and the women in the study. A theme that is argued throughout this thesis is the women of this study are Sophisticated Mediators - I will outline the origin of this concept and the redefinition from the original term. The final part is the Methods section in which I describe the rationale for qualitative interviews; how I located the women: who the women were and the time and place of the interviews. I also discuss the ethical issues and the steps I took to ensure confidentiality, the establishment of rapport, meaalofa (payment in kind), power relationships, the minimization of hierarchy within the interview process and finally the methods I used to code and analyse the women's stories.

\section{RESEARCH DESIGN}

To ensure the research process was designed and conducted in a sound and ethical manner, I drew upon the four-element model developed by Crotty (1998) i.e. epistemology, theoretical perspective, methodology and methods. The strength of Crotty's scheme provided a framework to conceptualise and clarify the foundation for this research project. Using the scheme as a guide, I was able to consciously and deliberately consider how the ideas underlying this project meshed together within the different layers, and ensure consistency between them (Crotty, 1998). 


\section{EPISTEMOLOGY}

The concept "epistemology" has been defined from various perspectives. Crotty defines it "as a way of understanding and explaining how we know what we know" (1998, p. 3) and also it is "the theory of knowledge embedded in the theoretical perspective and thereby in the methodology". Denzin and Lincoln (2000, p. 157) state that: “epistemology asks, how do I know the world? What is the relationship between the inquirer and the known? Every epistemology ... implies an ethical, moral stance towards the world and the self of the researcher" (Crotty, 1998). Epistemology is how we know what we know, it is a "theory of knowing" (Patton, 2002).

Indigenous epistemology, as Kwara'ae researcher David Gegeo defines it, is indigenous knowledge, an integration of "traditional" and "introduced" knowledge (Gegeo, 1998). In relation to the Kwara'ae people's theorising about rural development, Gegeo specifies that indigenous epistemology refers to a "cultural group's ways of thinking and of creating and reformulating knowledge using traditional discourses and media of communication (e.g. faceto-face-interaction)" (1998, p. 290). Gegeo provides a circular meaning to this process: "Indigenous epistemology guides the social construction of indigenous knowledge, and indigenous knowledge is the result of the practice of indigenous epistemology" (Gegeo, 1998, p. 290). An example relating to this study: women learn from mothers, sisters, aunts and midwives that breastfeeding is beneficial for both mother and baby. As these people are important advisors to the women and because they have experienced motherhood themselves, women are more likely to consider breastfeeding their babies because of the beneficial experiences of these important others. This is similar in nature to the concept by Vygotsky known as "scaffolding" (as cited in Bird, 2004) when inter- generations learn from each other with the elder generation with the greater knowledge supporting the younger generation. Over time that support reduces as competency occurs from the learner.

In an attempt to contextualise the definition of Gegeo's indigenous epistemology and how it can be understood within the context of this study, I provide the case of New Zealand-born Samoan women and their knowledge of pregnancy and motherhood. The indigenous epistemology of the women in the study (all New Zealand-born, most first generation) is constructed from the worldview (or position) of those who have influence in their lives i.e. family (mother, aunt, grandmother), culture and tradition and the women's own experiences growing up in New Zealand which has been moulded by their connection with school, friends 
and work. So, the indigenous knowledge of pregnancy and motherhood may be a combination of both traditional knowledge and knowledge they have gained growing up in a Western society.

In addition to Gegeo's indigenous epistemology, a complementary epistemological framework informing this research is Social Constructionism. This has been described by Sarantakos (2005) as relativist in that some element of experience or culture is relative or dependent upon some other element or aspect.

The experience of pregnancy, childbirth and early motherhood is affected by societal attitudes or cultural beliefs, whether traditional or Western. For example, within Samoan tradition, a pregnant woman's mother is an essential participant during a woman's pregnancy, childbirth and post-birth experience. She is involved at the very outset in teaching the pregnant woman about taboos, traditions and customs within the realms of Samoan culture, this knowledge will have been passed to her from her own mother, grandmother and possibly greatgrandmother; this is the spirit of fa'aSamoa. Pregnancy and childbirth are phenomena; they take place within a social context and have meaning for Samoan families within their social world (Macpherson \& Macpherson, 1990; Ngan-Woo, 1985). Accessing knowledge about pregnancy from the "Western" framework (i.e. the maternity health system) also plays an influential role in how women construct knowledge about pregnancy, childbirth and the transition to motherhood. Construction of a merged knowledge as discussed by Gegeo or “cultural fusion" as coined by New Zealand-born Samoan researcher, Anne Marie Tupuola, in her description of the emergence and increase of blending between the Western and Samoan worlds of Pacific youth (Tupuola, 2006, p. 298). This is particularly relevant for the women of this research who, I would argue, synthesise knowledge from two separate systems but mediate between the two to construct their own understanding and knowledge of their pregnancy, childbirth and motherhood.

The collective basis of social constructionism is useful for describing experiences and knowledge systems of peoples outside of the dominant paradigm (Ladson-Billings, 2000, p. 260). The focus includes "the collective generation (and transmission) of meaning" (Crotty, 1998). Different people may construct meaning in different ways, even in relation to the same phenomena (Crotty, 1998, p. 9 ), such as pregnancy and motherhood. For example, although people of Samoan descent are a minority in New Zealand, over half of this group is New 
Zealand-born. Their knowledge systems are unique in that they are embedded in a culture and tradition which is influenced by a variety of other societal groups. Social constructionists are interested in accounts that honour and respect the community of voices; telling our own story is, in fact, the story of our culture - its many voices heard in what we say (Crotty, 1998, p. 64). The emphasis is on capturing and honouring multiple perspectives of that story (Patton, 2002).

Furthermore, Crotty states: "meaning is not discovered, but constructed ... culture has a hold on us: it shapes the way in which we see things (even the way in which we feel things!) and gives us a quite definite view of the world" (1998, p. 58). Samoan people's view of the world is inspired by their surroundings and their cultural belonging. The traditional and cultural influences (for example church, cultural customs, and extended members of the family) maintain a strong influence in the construction of meaning that Samoan people have about pregnancy, childbirth and early motherhood. In turn, this can have an impact on the choice of support systems accessed or in the way experiences about pregnancy and post-birth may be portrayed.

The underpinning objective of this research was to understand and identify factors that affected the health and wellbeing of New Zealand-born Samoan women captured from the stories the women themselves constructed. The construction of factors affecting the women should be articulated by the women themselves. Therefore, I decided to conduct this research based on a person's lived experience rather than on the expert knowledge (for example midwives or maternity organizations) currently available to the women in this project; therefore, expert knowledge was not sought nor detailed in this research.

\section{THEORETICAL FRAMEWORK}

It was important for the methodological framework of this research project not to be limited by a unilateral approach, but allow a range of interpretative paradigms (for example feminism, social constructionism and Pacific studies) to be drawn together to explore the maternal health of New Zealand-born Samoan women.

\section{Bricolage}

The researcher-as-bricoleur draws on different methodologies and ideas from across disciplines to develop processes and methods that will get the job done. The decision 
about which tools or practices to use depends on the research questions and the research setting and cannot be decided in advance (Asiasiga, 2007, p. 95).

Research is shaped by assumptions and also by the principles and concepts described in theoretical perspectives. The approach to this study was informed by various theories and evolved during the progress of the thesis into one that is bricolage in spirit. The French word bricoleur refers to a "handyman or handywoman who makes use of the tools available to complete a task" (Harper, 1987 as cited in Denzin \& Lincoln, 2008). Making use of the "tools available" in search of understanding ensures that "qualitative research draws on different theoretical perspectives to make sense of information collected from participants. It is difficult to stay within one theoretical framework if what is found seems to be explained by another" (Asiasiga, 2007, p. 95).

Similar to the concept of bricolage Wesley-Smith insists on combining the methods and approaches of several disciplines in his quest to advocate a new emphasis on interdisciplinary forms of scholarship within Pacific studies. Wesley-Smith posits two aspects: (1) that societies for all their connections, political, cultural, economic, social, linguistic, or spiritual features cannot be situated under one defined discipline (Wesley-Smith, 1995, p. 128). The second aspect is that becoming interdisciplinary "recognises the key roles of creativity, subjectivity, and poetics in the 'science' of interpreting and representing the social world" (Wesley-Smith, 1995, p. 128). This is important as it allows for the incorporation of multiple voices within the narratives (as in feminist research), and provides space for reflexivity of researchers while acknowledging the contingent and open-ended nature of inquiry (WesleySmith, 1995).

Furthermore, Roland Barthes argues that "to do something interdisciplinary it's not enough to choose a 'subject' (a theme) and gather around it two or three sciences. Interdisciplinarity consists in creating a new object that belongs to no-one" (as cited in Wesley-Smith, 1995, p. 123). Crotty recognises the significance of a research question being a useful starting point and argues that "we plan our research in terms of the issue or problem or question" (1998, p. 13) rather than fitting the question around an epistemology or a method. 
In her thesis examining the way in which migrant Samoan women gave birth in Wellington, Patricia Donnelly adopted an "eclectic" approach converged themes from sociological, anthropological and psychological literatures which she felt contributed to developing a more robust and solid framework of analysis (Donnelly, 1992). The bricolage/interdisciplinary approach allowed me to explore multiple views - the empowerment of women, reflexivity, insider/outsider dichotomy, power relationships, the situated lives of women and the cultural synthesise.

\section{Feminism}

For a woman-centred thesis, feminist theory is an obvious resource, but defining feminism is anything but straightforward as there are so many schools of thought. Campbell and Wasco (2000) describe four main types of feminism: liberal feminism, radical feminism, socialist feminism and womanism, whilst Putnam Tong (1998) suggests ten types of feminism, two of which have already been mentioned, liberal and radical, in addition to Marxist, psychoanalytic, gender feminism, existentialist, post-modern, multicultural, global and ecofeminism; in addition Black feminism (Hill Collins, 1989).

A feminist approach considers multiple constructed realities that are shaped by society, politics, culture, economics, ethnicity and gender. Within feminist theory, facts are inextricably linked to values; certain societal groups have more power than others i.e. the oppressor over the oppressed (Guba \& Lincoln, 2000). The ultimate goal of feminist research is to capture women's lived experiences in a respectful manner that ensures the legitimacy of their voices as sources of knowledge. A commitment to feminist values and processes of research was the motivating factor guiding this research process. Fonow and Cook (as cited in Crotty, 1998, p. 175) suggest that "carefully designed research grounded in feminist theory and ethics is more useful to understanding women's experiences than an allegiance to any one particular method as more feminist than another".

\section{Feminism within a Samoan context}

The philosophical stance for informing the methodology of this study developed over time and draws from a variety of feminist theories, namely black feminist thought (Hill Collins, 1989, 1990, 1998), womanism (Campbell \& Wasco, 2000), and aspects of the cultural concept fa'aSamoa (the Samoan way) (Ngan-Woo, 1985). 
This study is concerned with ensuring the stories of New Zealand-born Samoan women are not placed solely within a New Zealand cultural framework, nor that of a purely Samoan framework, rather a creation of their own standpoint that holds value in both cultural worlds. Womanism considers that the intersection of multiple forms of oppression (including class, ethnicity and gender) affect women in different ways (Campbell \& Wasco, 2000). This is exemplified in Black Feminist Thought which emerged from the oppression of AfricanAmerican women in their struggle to create their own standpoint. This encourages AfricanAmerican women to "value their own subjective knowledge base" and to self-define their standpoint as African-American women (Hill Collins, 1989, p. 750).

Defining what it is to be a New Zealand-born Samoan woman is to include all aspects of what contributes to that identity. The concept of fa'aSamoa commonly referred to as "the Samoan way" (Ngan-Woo, 1985) is defined as keeping in accordance with Samoan tradition and customs. Rather than being a fixed and rigid concept, one of the fundamental elements of fa'aSamoa is its fluidity which enables it to shift in "meaning" in different contexts. This is an important point in that fa'aSamoa principles are a part of what make Samoan people unique and it is embedded within the Samoan culture. However, it bears in mind that the adherence to, or acknowledgement of, fa'aSamoa for New Zealand-born Samoan women is varied; the extent of importance depends heavily on the diversity of the backgrounds of the women living in New Zealand (Tupuola, 1998). Furthermore, the level of exposure to, and understanding of, fa'aSamoa was different for each woman in this study.

Pacific women have traditionally viewed and received Western feminism with much scepticism, suspicion, criticism and rejection, not accepting it as a concept that fits within their own social and cultural context. Within Samoan tradition the "family is the principal focus of identity and social location" (Fairbairn-Dunlop as cited in Stewart-Withers, 2007, p. 146). Within this context therefore, Western feminism is seen as a threat to what has been argued is the Samoan women's traditional seat of power - the family (Marsh, 1999).

In exploring the relevance of Western feminism for Pacific women, Marsh attempts to unpack the philosophy of feminism and contextualises texts from well known Pacific writers, poets, and educators to define the meaning of the term feminism within a Pacific context (Marsh, 1999). One of the major issues Marsh illustrates is the potential clash between the indigenous view of "communalism" and Western values based on "individualism" (Marsh, 1999, p. 670). 
This subject, although not new, continues to be a salient point highlighting what differentiates Pacific people's worldview from those of the Western world; concisely illustrated as "the individual ' $I$ ' is almost inseparable from the communal 'we'” (Marsh, 1999, p. 675; Tiatia, 2003). In addition, others have described "the Samoan self" as having meaning only in relationship with other people, rather than as an individual. The "self" could not be separated from the "va" which means relational space/negotiated space that occurs between two people, or groups of people (Marsh, 1999; Mila-Schaaf \& Hudson, 2009; Tamasese, et al., 2005). This is the crucial element of what is considered being "Samoan".

What emerged from the Pacific literature was a sense that "many Pacific women have always engaged with feminism", even if the label had been rejected. Thaman notes: "I was a feminist long before the movement came along, and my grand-aunts were feminists before the word feminist became part of my vocabulary" (Woods, 1997, as cited in Marsh, 1999, p. 677). The women of Samoa play an integral part within society at large. The setting up of women's committees by pastors' wives in the 1930s to organise church affairs brought them into public decision-making roles for the first time (Fairbairn-Dunlop, 1998). From these committees the komiti tumama (village health committees) were formed which strengthened the role for wives even further (Fairbairn-Dunlop, 1998). This was an important step toward the visibility of women in roles that were historically the role of men.

A Samoan view of feminism perhaps differentiates itself from the more Western view in that it is a more holistic standpoint for Samoan women. What is labelled as feminism in the Samoan context is seen as beneficial for not only women but their children, families and the community rather than being beneficial at an individual sense. This is illustrated in the following statement of the Honourable Safuneituuga Paaga Neri, at the $49^{\text {th }}$ session of Commission on the status of women in New York, in which she quoted an ancient Samoan proverb: "E au le Inailau a Tamaitai", which means "the legacy of women is one of total achievement". Indeed, the high status of women has long been recognised in Samoan culture and traditions. "When women succeed, their families succeed and society prospers; this is the Samoan way" (Safuneituuga, 2005). 


\section{METHODOLOGY}

Drawing on the contents of the preceding discussion of epistemology and theoretical frameworks, the research methodology for this thesis can be described as feminist phenomenological social constructionist.

The women of this study were exposed to two, not quite opposing, cultures. The social constructionist perspective helps to understand how meaning is taught in a complex and subtle process of enculturation and how this shapes thinking and behaviours throughout (Crotty, 1998). Within feminist methodology, deconstruction of oppression and providing a platform for the oppressed (for example women, youth, minority groups with no power) to be heard is particularly significant in social research. A phenomenological approach focuses on capturing and describing how people experience some phenomenon; in this case, how Samoan women experience pregnancy and post-birth - their perceptions, descriptions, feelings, judgements, how they remember it and how they make sense of it and talk about it (Patton, 2002). In essence, then, the methodology for this study is described as being a feminist phenomenological constructionist study i.e. presenting the voices of New Zealandborn Samoan women talking about the construction of pregnancy, childbirth and motherhood from their own experiences.

\section{Phenomenology}

Edmund Husserl is frequently cited as the founder of phenomenology which emerged around the turn of the twentieth century (as cited in Crotty, 1998, p. 79 ). The operative word in phenomenological research is "describe". The aim of phenomenological research is to describe as accurately as possible the phenomenon, refraining from any pre-given framework, but remaining true to the facts (Davidson \& Tolich, 2007); this approach focuses on each individual's own subjective experiences as she encounters those events (Patton, 2002) which in my research is the lived experience of Samoan women during late pregnancy and postbirth.

The application of phenomenology is concerned with the lived experiences of people (Kvale, 1996) and "what we directly experience" (Crotty, 1998, p. 79). Crotty also states: "phenomenological research emerges as exploration, via personal experiences, of prevailing cultural understandings" (1998, p. 83). My research is an attempt to find the meanings that New Zealand-born Samoan women ascribe to their experience around pregnancy, childbirth and motherhood. Focus is therefore also on the social world of Samoan women i.e. a world 
that is constructed by the culture they are part of, in this case the two cultures of traditional (Samoa) and Western (New Zealand). It is important to understand their experiences and their interpretation of the world. According to Patton (2002), in order to really know and understand, it is important to experience the phenomenon as directly as possible either through participant observation or in-depth interviewing. While participant observation is one way of observing how one interacts or participates with their social world it does not provide a world-view perspective from the participant. Furthermore, qualitative research is interested in the reconstruction of events or expectations of events i.e. asking women to recount when they found out about their pregnancy or to articulate their expectations of motherhood.

Participant observation cannot reconstruct events or know about previous events.

However, "living" an experience takes Patton's suggestion of experiencing a phenomenon as directly as possible one step further, and one that is appropriate for this research. I believe that to share the same phenomenon as those you are researching can enhance one's understanding. For example, as are the women in this research, I am a New Zealand-born Samoan woman of migrant parents who experienced my first pregnancy in my early twenties in New Zealand. I had access to similar knowledge systems around pregnancy and identifying with the women in the study at this level added depth and breadth to my understanding of their experiences. The involvement of the researcher within the research is a hotly debated issue yet, in this instance, I was reassured by the knowledge that my visibility in the research project connected my experience with that of the women involved in the research project (Reinharz, 1992).

\section{Insider/Outsider}

Crotty (1998) emphasises that researchers must prevent, or at least minimise, the imposition of the researcher's presuppositions and constructions on the data. Husserl contended that bracketing, setting aside preconceived notions, enables one to describe the phenomenon under study without tainting the data (Byrne, 2001). For example, to understand the meaning of pregnancy and post-birth for Samoan women, Husserl's approach would assume the current investigator would bracket everything she knows personally about pregnancy, childbirth and pregnancy to enable the emergence of the core meaning of pregnancy and postbirth for Samoan women. However, this assumes one can separate personal knowledge from life experiences - thus adopting an objective viewpoint. 
My role as a researcher and my self-defined ethnicity as a New Zealand-born Samoan placed me in a unique position of insider as well as outsider. The status of insider, outsider or outsider-within, is an issue for any researcher in the social sciences (Hill Collins, 1986) and the complexity of the insider/outsider concept has been commented on by many researchers (Asiasiga, 2007; De Andrade, 2000; Hereniko, 2000; Smith, 1999). Hereniko posits the insider/outsider concept as a distinction between "knowing" and "living/being". Intuition, emotion and sensibility are things that an outsider can never fully grasp: they are things that are "acquired through early socialisation in the formative years" (Hereniko, 2000). However, being an insider and simply sharing the same ethnic origins or gender is sometimes not enough when researching groups. The insider status is not simply granted or achieved but created through an ongoing process of evaluation at multiple levels by both the researchers and participants, particularly with minority communities (De Andrade, 2000; Hereniko, 2000).

The changeability of the insider/outsider/outsider-within concept provided both advantages and disadvantages as the research progressed. The variability of this concept was summed up by Lanuola Asiasiga, a New Zealand-born woman herself, during her research with Pacific women when she stated that:

Everything is relative and no-one is wholly an outsider or insider. Relationships are constantly shifting so that even within a group where a researcher is an insider there will be some factors that create distance and others that create connection (Asiasiga, 2007, p. 91).

This is an important observation that allowed me to be comfortable that I did not need to solidify a particular position of either being an insider or an outsider. It also helped me to be mindful of subtle changes that can occur, even during an interview, and of the need to adapt to each situation to ensure the connection was maintained.

Conducting research with New Zealand-born Samoan women was reflective of who I am, as a person. Although our experiences are unique, I considered myself as an insider. However, my role as a researcher attached to a university and also being considerably older than the eldest woman (17 years older than the eldest woman interviewed) immediately marked me as an outsider. 
During the recruitment process, as an insider I had access to people who were willing to help recruit my sample and, as a Pacific researcher, I was part of a Pacific network which assisted greatly by publicising the research within the Pacific community. During the interviews I found myself constantly weaving between insider and outsider. For example, I felt like an insider when the women were sharing their experiences about: living or negotiating between two cultures and what that brings to the experience; the prospects of becoming a mother for the first time; the expectations as children of our migrant Samoan parents; the wonder and challenges of pregnancy; the stories of birthing; utilisation and expectations of the maternity system and finally, being part of a large Samoan family. As the research developed I considered my insider/outsider position to be constantly changing or "fluid" (De Andrade, 2000) dependent on the context. It was also interesting to note it was not only my own perspective but also the perspective of the women I interviewed that dictated my insider/outsider status (Beoku-Betts, 1994). De Andrade (2000) addressed the insider/outsider debate from her experiences conducting research on the construction of racial and ethnic identity in the Cape Verdean American community of south-eastern New England, noting that:

Participants' assessment of my group membership or insider status appeared to include an assumption that I shared their knowledge and experience (p. 269).

One of the women from my research described her experience of negotiating between what her mother felt was culturally acceptable (e.g. a fofo) but which she herself felt was inappropriate (e.g. "no fofo - it's not certified"). This was followed by the woman commenting "you know what they're (Samoan mothers) like" followed by laughter. The comment assumed we shared a definition of Samoan mothers, without the need to articulate it further. This illustrates the insider status afforded to me by this particular woman, in that particular situation. In addition, because I acknowledged I knew what she had meant, it helped to build a rapport and develop the relationship. This also resonates with what De Andrade described as a "locating process" i.e. participants use several evaluation techniques to try to link the researcher to their own social world (p. 281), and by doing this the participants soon regard the researcher as an insider.

Yet the perception that an insider possesses more privilege over an outsider is seen as questionable. Baca Zinn (Baca Zinn, 1979) experienced difficulties as an insider researcher within the Chicano community and found that, although she was an insider by ethnicity, 
because she was not part of the particular organisation or community that she was researching, she was still viewed as an outsider. The purpose of her research was to study marital roles, marital power and ethnicity in Chicano families. To locate families Baca Zinn fully participated in community programmes such as a local sewing class, and through this participation felt she was able to acquire a place in the community and therefore develop relationships with the women, something that may have been difficult to achieve had she not involved herself within the community (Baca Zinn, 1979).

Insider researchers are faced with different sets of difficulties as opposed to outsider researchers. Linda Smith (1999) refers to insider research as having to be ethical, respectful, reflexive and must be just as critical as outsider research. She also points out the need to be "humble" rationalised by the fact the researcher belongs to the community and therefore abides by a different set of roles, relationships, status and position, whereas outsiders do not. Furthermore, situating oneself as an insider has far greater impact on the community and families of the insider than does being an outsider. In reference to insider researchers Linda Smith states "complexities of insider research can be mediated by building support structures" (p. 139) which might be support groups or could involve community or academics, or elders of the community to guide the researcher through the research journey.

The concept of "outsiders-within" was first coined by Patricia Hill Collins in describing "the location of people who no longer belong to any one group" (Hill Collins, 1986), referring in particular to the positioning of Black women. The concept was useful for Beoku-Betts in her experience studying Gullah women and their communities, for while she herself identified as an insider being a Black researcher, she also self-reflected on her status as a cultural outsider. The outsider-within construct helped Beoku-Betts to understand some of the contradictions in her own experience during her research. However, the use of the term decontextualised the original definition as described by Hill Collins. In her article "Reflections on the Outsiderwithin" she aimed to redress the meaning of outsider-within back to the context of defining a group rather than the current use as a "personal identity category". Hill Collins is clear that the construct outsider-within refers to "how a social group's placement in specific, historical context of race, gender, and class inequality might influence its point of view on the world" rather than the personal identity category which has been, and continues to be, commonly used by many researchers (Hill Collins, 1999, p. 85). Furthermore, Hill Collins (1999) argues the personal identity redirects attention away from the social hierarchies of race, class, and 
gender that created the social location of outsider-within in the first place (p. 86). This is an important point made by Hill-Collins in reference to how other researchers often redefine and use the concept. Her reminder of the original purpose modified my own thinking around adopting the outsider-within term and how as a researcher I found and interpreted the meaning of the concept. During the early stages of the research I embraced the outsiderwithin term as an individual researcher in my relation to the women in this study. However, after reading Hill Collins definition and contextualisation of the term I realised the outsiderswithin were in fact the women as a group i.e. they were New Zealand-born Samoan women living in New Zealand and therefore were outsiders-within in relation to New Zealand society and culture as a whole.

Feminist standpoint theory posits that for less powerful groups to survive they must "be attuned to the culture of the dominant group" and that individuals within the group "have the potential for a more complete and less distorted view of social reality precisely because of their disadvantaged position" (Nielsen 1991 as cited in Campbell \& Wasco, 2000, p. 781). This standpoint, however, must be developed by appropriating one's experiences through intellectual political struggles against gender, race, class, and sexual orientation inequalities (Allen \& Baber, 1992; Collins, 1987, 1989; Harding, 1987; Hartsock, 1987, 1998 as cited in Campbell \& Wasco, 2000, p. 782). In reference to this standpoint, Campbell and Wasco (2000, p. 781) refer to this as "double-vision" by which groups who live and are influenced by both the dominant culture and their own culture i.e. individuals of marginalised groups can develop a type of double vision which is interpreted as a "more comprehensive understanding of social reality" (Hartsock, 1987, 1998; Westkott, 1990 as cited in Campbell \& Wasco, 2000, p. 781). This aligns to what Hill Collins terms "sophisticated mediators" in which she refers to the child rearing practices of black mothers from an article by Janice Hale (1980) in suggesting that "effective Black mothers are sophisticated mediators between the competing offerings of an oppressive dominant culture and a nurturing Black value-structure" (Hill Collins, 1986, p. 183).

\section{Sophisticated Mediators}

This term, which I found mid-way through the analysis of my data, immediately resonated with me in regards to the behaviour of the women and how they were maintaining or acquiring resilience in the face of adversity. Gegeo's definition of indigenous epistemology i.e. being a culmination of two knowledge systems (as described earlier), legitimates the term 
of Sophisticated Mediators in the spirit of how the term/concept is defined within this thesis. The term Sophisticated Mediators, as described in the Introduction chapter, is a modification of the definition provided by Hill Collins. The women possessed an ability to identify their needs before selecting appropriate solutions from the offerings of both their Samoan cultural system and their New Zealand culture - at both a social and individual level. Mediating successfully ensured they were able to sustain or obtain resilience against risk factors that helped them to make decisions that led to positive outcomes during pregnancy, childbirth and motherhood.

Standpoint theorists issue a challenge to find groups on the margins of social structures and actively engage them in describing their experiences and perceptions (Campbell \& Wasco, 2000). Practically, feminist standpoint research utilises a variety of methodologies (for example both qualitative and quantitative approaches) to engage research participants (typically members of oppressed groups) in reflection on how their gender, race, social class, and sexual orientation shape their experiences in the social world. For example: How does a woman of Samoan descent living in New Zealand experience pregnancy? What health systems does she draw from most during her transition to motherhood - traditional, Western or both? What support systems are accessible for these women - formal and informal? How does being a minority woman influence their experiences? In addition, researchers conducting feminist research must not only reflect upon but also acknowledge how their own social group status influences their interpretations of their data (Allen \& Baber, 1992 as cited in Campbell \& Wasco, 2000). For example: how would my status as an older Samoan woman affect my interpretation of the data of this young group of women? What are some of the issues that I should reflect on when analysing the data?

\section{METHODS}

It is important to acknowledge a distinction between methodology and methods. Harding (as cited in Pere, 2006) defines research methodology as "a theory and analysis of how research should proceed", whereas a research method is "a technique for (or way of proceeding in) gathering evidence". The methods which shape this research are fundamentally qualitative.

A qualitative approach allows the investigation of phenomena in depth; qualitative research is interested in the interpretation and contextualisation of the data; it generates theory and is inductive; personal involvement and partiality (subjectivity) is valued; it allows for more 
flexibility in that several interviews can be conducted at different times; it also draws heavily on impressions, descriptions, and quotes (Tolich \& Davidson, 2003). Therefore, a qualitative approach was the best research methodology given the experiences of the women was the core interest of this research.

Some of the limitations of qualitative research are that it is time consuming, can be expensive, can sometimes produce problems around anonymity and safety for both the researcher and participants (Sarantakos, 2005) and is not generalisable. Qualitative research can be time consuming and is much less structured than quantitative research, with greater emphasis on the participants' own perspectives and interest in their points of view. This approach allows the participants to explore other areas they consider important to them and can be very insightful for the researcher (Sarantakos, 2005). Therefore, set times are not always appropriate and this is particularly true with one-to-one interviews, or multiple interviews, as conducted with this research where each woman was required for two interviews, the longest being just over two hours. This was time consuming both for the preinterview preparation and the interviews themselves. Also, considerable time was spent with the women before the formal interview commenced (getting to know each other) as well as after the interview (debriefing, questions answered).

Conducting individual interviews constitutes a financial expense as meaalofa (voucher), food, transport, postage costs, recording equipment and transcribing costs were required to conduct the interviews. The issue of anonymity directly relates to the participants not being able to be identified by their responses. Individual interviews with all the women were face-to-face and transcribed later by me who obviously, as the interviewer, was fully aware of each of their responses. Therefore, individual interviews, while confidential, can never be truly anonymous, particularly for this study as the sample was small (Sarantakos, 2005).

Confidentiality, which is often confused with anonymity, is when the researcher is fully aware of the responses but promises not to make the connection in public. This was upheld by my own code of ethics as well as the strict ethical guidelines set out by the University under which this research was undertaken, and which was communicated to the women to reassure them about the safety of participating in this research. The limitations as discussed above are also discussed in the ethical section of this chapter. 
Generalisability is not the intended direction for this thesis, and it is acknowledged that the knowledge produced from qualitative research may be unique to the few participants in the research study (Sarantakos, 2005), hence the lack of generalisability. It is anticipated the findings of this thesis will add to the knowledge and aid in any future investigation.

\section{Rationale for individual interviews}

The methods or "techniques or procedures used to gather and analyse data related to a research question" (Crotty, 1998, p. 3), are framed within a phenomenological methodology. The aim was to arrive at the most effective design that informed the epistemology and perspective, as well as allowing for rich descriptions to emerge.

Consideration of the appropriate method was heavily influenced by other researchers who have worked with the Pacific community (Asiasiga, 2007; Tamasese, et al., 2005; Tiatia, 2003; Tupuola, 1998). The methods I considered needed to be appropriate for the intended participants whilst also ensuring those sharing their experiences were doing so in an environment that was both safe and culturally sensitive. Furthermore, in line with the feminist approach, Reinharz (1992) advocates interviewing as it offers researchers "access to people's ideas, thought, and memories in their own words rather than in the words of the researcher" (p. 19). A narrative approach allows the participant to tell of their experience, using their own words to describe their situation, emotions and perceptions thus giving an authentic insight into their own experiences. Therefore, I considered conducting both focus groups and individual interviews.

Focus groups are groups of people brought together for the purpose of the study and involve various participants guided by the researcher. The aim is to facilitate group discussion, brainstorm a variety of issues and to establish a mechanism of opinion formation (Sarantakos, 2005). It is a technique that is widely used in marketing and social research. Within a qualitative research model, while participants interact with each other, group discussion offers access to the construction of meanings, the breadth and variation of those meanings, and the way in which the group renegotiates them (Sarantakos, 2005; Tamasese, et al., 2005). However, the success of focus groups relies on the interplay between the facilitator, the situational context, the group, and the topic. Limitations of a focus group discussion include: participants may hide their real opinions or feelings; one or two participants may dominate 
the discussion; some participants may not participate in the discussions due to shyness or intimidation; recording of the data may be problematic due to overlapping discussions; participants may not want to divulge personal feelings (Tolich \& Davidson, 2003). For some women pregnancy and post-birth may be experiences they may not want to share in a group, particularly if that experience was negative. For some Pacific women, talking about the physical body can be a very personal issue that they may prefer not to discuss in a group setting (Tupuola, 1998). Therefore, considering this research was focused on women's own experiences and for the reasons as discussed above, a focus group was considered an inappropriate tool to utilise for this particular study.

Face-to-face individual interviews, or "verbal questioning" is the most common method of data collection (Sarantakos, 2005). The concept of narrative interviews was developed by Shutze (Sarantakos, 2005) and was employed in this study. Narrative interviews focus on stories that are told by the subjects: on the plots and structures of their accounts (Kvale \& Brinkmann, 2009). This may evolve naturally throughout the interview or be elicited or prompted by the interviewer. Such prompting allows the interviewer to introduce questions about specific episodes or happenings in an attempt to structure coherent stories (Kvale \& Brinkmann, 2009).

The rationale for using narrative interviews was to gain intimate rich data using a semistructured interview schedule. This ensured that the essence of the key issues the women wanted to address was captured: that is, the participant defined the themes they deemed important whilst simultaneously allowing further areas of interest relevant to this study to be probed. The employment of this interviewing technique was supported by other Pacific researchers who contend that the narrative approach is a suitable method to address "sensitive" issues within Pacific communities (Tamasese, et al., 2005; Tiatia, 2003).

\section{Data Collection}

\section{The women who participated in this study}

Samoan women born in New Zealand living in Wellington were participants in this study. The inclusion of Samoan-born women in Wellington and Auckland was initially planned as it would have added breadth to the research however, logistically and financially, this proved to be unachievable because two interviews were required from each woman, the transcripts needed to be hand-delivered to each woman and, finally, each woman needed to be included 
in a brief interview during the validation phase. The women in this research were first-time mothers living in the Wellington region who were in their last trimester of pregnancy. All of the women interviewed were of Samoan descent and born in New Zealand with English being their primary language. All the women who were interested in taking part were either called on the telephone or emailed. As part of the criteria to participate, all of the women approached agreed to take part in both Phase 1 and Phase 2 of the data collection. In the original design it was planned to recruit eight women for this study. However, due to this study having two phases (last trimester and post-birth) and the length of the study (1 year) the logistics of recruiting eight women during Phase 1 while having enough time to allow for the Phase 2 interviews proved to be more difficult than anticipated. Of the seven women who contacted me to participate in the research, two were in their last trimester of pregnancy but were ineligible as this was not their first pregnancy whilst another had given birth before the interview could take place. Therefore, only four women met the criteria and were therefore eligible to take part. At Phase 1 of the interviews the four women were aged between 20 and 29 years and all self-identified as New Zealand-born Samoan. Table 1 and Table 2 below provide brief facts about the women. Please note - pseudonyms are used.

Table 1: Information about the women - ANTENATAL PERIOD

\begin{tabular}{|l|l|l|l|l|l|l|}
\hline & Age & Marital status & Work & $\begin{array}{l}\text { Contraceptive } \\
\text { use }\end{array}$ & Pregnancy & Lives with \\
\hline Alma & 20 & $\begin{array}{l}\text { Married } \\
(1.5 \text { years })\end{array}$ & Paid & No & Unplanned & $\begin{array}{l}\text { Husband \& extended } \\
\text { family }\end{array}$ \\
\hline Geraldine & 20 & $\begin{array}{l}\text { In a } \\
\text { relationship } \\
(2 \text { months })\end{array}$ & Paid & No & Unplanned & Parents \\
\hline Elegi & 20 & $\begin{array}{l}\text { In } \\
\text { relationship } \\
(6 \text { months })\end{array}$ & Paid & No & Unplanned & Aunt \& Uncle \\
Harried & Paid & Years $)$ & Planned & $\begin{array}{l}\text { Husband \& step- } \\
\text { children }\end{array}$ \\
\hline
\end{tabular}

Source: (Churchward, 2010).

Table 2: Information about the women - POSTNATAL PERIOD

\begin{tabular}{|l|l|l|l|l|l|l|l|}
\hline & Age & Marital status & Work & $\begin{array}{l}\text { Perceived } \\
\text { birth } \\
\text { exp. }\end{array}$ & Feeding & $\begin{array}{l}\text { Satisfaction } \\
\text { with } \\
\text { midwife/ves }\end{array}$ & Lives with \\
\hline Alma & 21 & $\begin{array}{l}\text { Married } \\
(1.5 \text { years })\end{array}$ & $\begin{array}{l}\text { Unpaid } \\
\text { at home }\end{array}$ & Difficult & Breast & No & Husband \& baby \\
\hline Geraldine & 21 & $\begin{array}{l}\text { In } \\
\text { relationship } \\
\text { (2 months })\end{array}$ & $\begin{array}{l}\text { P/time } \\
\text { paid at } \\
\text { home }\end{array}$ & Okay & Bottle & Yes & Parents \& baby \\
\hline Elegi & 21 & Single & Study & Difficult & Breast & Yes & Aunt, Uncle \& baby \\
\hline Henrietta & 30 & $\begin{array}{l}\text { Married } \\
(3 \text { years })\end{array}$ & $\begin{array}{l}\text { P/time } \\
\text { paid } \\
\text { work }\end{array}$ & Okay & Breast & Yes & $\begin{array}{l}\text { Husband, } \\
\text { children \& baby }\end{array}$ \\
\hline
\end{tabular}




\section{Locating the women}

Purposeful sampling and snowballing (Patton, 2002, p. 43) was used to locate women to take part in the research. According to Patton "the logic and power of purposeful sampling derive from the emphasis on in-depth understanding. This leads to selecting information-rich cases for study of in depth information" (p. 46). The snowballing technique is when participants are asked to suggest anyone they know who might be interested in participating in the research. Several sources were used to help find women for this research, personal networks, email distribution to Pacific networks, midwifery and antenatal services.

The recruitment process was labour intense and very detailed. Some of the recruiting time was during the lead up to Christmas which also turned out to be problematic, considering the busyness of the period. The process was designed to capture women from all over Wellington; however, it did not yield many women and may have benefitted from a modification to the process earlier on. Of the four women who were invited to participate, one woman was recruited through another potential participant; two women were recruited through another project I was involved with but asked to be part of this particular research instead. The last woman responded to the flyer (see Appendix) sent to one of my associates. All the women were given a brief outline of the research project either verbally or in written form (information sheet) and were then contacted by telephone. The number of women recruited was smaller than was proposed and while according to Patton (2002) one cannot generalise from very small samples it can open up a "new territory for further research" (pg 46). Having smaller numbers of participants allows for relationships to become closer between researchers and participants and therefore enhances a "validity of fine-grained, indepth inquiry in naturalistic settings" (Crouch \& McKenzie, 2006, p. 483).

\section{Interviews}

For both phases, semi-structured, face-to-face individual interviews were conducted with four women who identified as New Zealand-born Samoan. The first interview (Phase 1) was conducted with the women who were in their last trimester of pregnancy (between 26-40 weeks pregnancy). At the second interview (Phase 2) I met up again with the women when their babies were 6 months; 8 months; and two babies who were 10 months old. Originally, the Phase 2 interviews were to be carried out 4-6 months after each woman had given birth. However, ethics approval was sought and approved for the extension of the post-birth period for up to 12 months as there was a delay in starting Phase 2. This proved to be more of a 
suitable time as Brown et al (as cited in Kerslake-Hendricks, 1998) found that following the birth of a baby there may be a "halo effect" influencing women's perceptions of birth i.e. women are less likely to be critical of their situation. Kerslake-Hendricks comments that "a more critical account of their experiences is given after a six month lag, during which women have had time to reflect on what actually happened" (1998, p. 24).

\section{Time and place of interview}

There were no expectations about the minimum or maximum length of an interview however all the women were informed that each interview was likely to take between $1-1 \frac{1}{2}$ hours. In actuality, the shortest interview was 41 minutes while the longest was recorded as 2 hours and 5 minutes. I interrupted this latter interview after the first hour to ascertain whether the woman was happy to continue, which she confirmed. The women were also offered their choice of venue for the interview i.e. at my office at the university, in their own home, or in the home of a work colleague of mine who lived close to the women concerned. Offering a choice of venue reduced any pressure on the women to host me in their own homes for the interviews and also ensured they could choose the setting where they felt most comfortable.

Each of the four interviews in Phase 1 were conducted in a different setting: one at my office; another relocated to the home of the participant's brother after discovering the cafe where the interview was to be held was closed; another at the home of my work colleague who lived locally; and the last was in the participant's home. The women chose the day and times of their interview; two were conducted mid-week and two on a Saturday and Sunday morning. Two women lived in their own homes and two in homes with their extended family. Two interviews were conducted in the participant's home when others were in the house and while I was initially concerned about how that situation could have negatively influenced the participant's freedom to express them; the women themselves however were unconcerned and appeared comfortable throughout the interview. Transport was an issue for only one woman but this was resolved by me collecting her to attend the interview and returning her home. This had the additional advantage of allowing the woman to be interviewed away from her home during a time her in-laws were also there.

In Phase 2, when the babies were aged six months or older, each woman chose to have the interview conducted in her own home. This may have occurred due to the fact that I had by this stage established a rapport with the women during Phase 1 and the level of trust established in the relationship encouraged the women to invite me into their homes. From a 
purely practical standpoint it also meant they did not have to leave the house with baby in tow or find a babysitter so they could attend the interview. Although two women had family members in the home at the time of the second interview, again this was not a concern for the women and for three of the interviews the baby was present.

\section{Ethics}

Information sheets and consent forms (see Appendices section) were developed for all the women involved in the research. The consent forms reiterated that participation in the research was entirely voluntary and that the women had the ability to withdraw their data from the project at any time prior to the final thesis being submitted. They also indicated how long the interview was likely to take (approximately $1-1 \frac{1}{2}$ hours), confirmed that all the information pertaining to the entire research project was to be kept confidential by the researcher and that no individual would be able to be identified from any research reports produced.

Confidentiality was one of the key ethical considerations when embarking on this research, particularly when the women were drawn from a relatively small population. The data therefore has been structured in a way that allows the identities of the participants and their families to remain confidential. The use of pseudonyms and initials (Richards \& Schwartz, 2002; Tiatia, 2003; Tupuola, 1998) minimises the likelihood of identification and is commonly used among qualitative researchers. Each woman was given a pseudonym together with the number of interviews e.g. Ellen(1) and the names of the children or partners referred to the women have also been changed i.e. the names of the partners are replaced with a capital letter only, while the child's name is replaced with a full name e.g. Lemmie. Other details such as the physical attributes of the babies that had the potential to identify the women were also excluded.

\section{Establishing Rapport}

Establishing a good rapport with the women at the outset was essential for the success of this research project. There were two phases involved in the data collection and failing to establish a strong rapport with the women in phase 1 may have alienated the women and also risked their continued participation at phase 2. Establishing trust and rapport was not restricted to the interview process itself but began from the first point of contact and continued through to the end of the research process. This led to creating a relationship with 
the women based on trust, flexibility and honesty (Campbell \& Wasco, 2000). During the first telephone contact with the women, which lasted no more than 10 minutes, I endeavoured to project an informal yet professional tone while maintaining an interest in the health of the women i.e. enquiring about their pregnancies, how they were feeling, the due date of their babies etc. This first contact provided the women with the opportunity to gauge my sincerity. They were able to ask questions spontaneously knowing that they could terminate the relationship at the end of the telephone call i.e. they held the power in the relationship. This first contact was crucial not only in confirming their consent to participate in the study but also to establish a sense of connection with the women and the beginning of a rapport that would continue throughout the interviews, which invariably occurred. I established an immediate rapport with the women due to the fact that I too was a mother and also a New Zealand-born Samoan and this rapport continued to develop with every contact.

During the down time between Phase 1 and Phase 2 interviews (up to 8 months in some cases) I posted hand written cards, emailed, telephoned or texted the women to maintain the relationship. One of the most important principles in maintaining and sustaining research relationships is reciprocity and this is particularly salient for Pacific cultures (Health Research Council of New Zealand, 2005) because Samoan culture emphasises reciprocity and generosity (Ngan-Woo, 1985) where demonstration should be by way of attitude and spirit, as well as in tangible ways (Health Research Council of New Zealand, 2005). Reciprocity was offered to each of the women at the end of each interview in monetary or material contributions, as a token of my appreciation but also, more importantly, throughout the interview in the form of personal information sharing in the spirit of the project. I was not comfortable with only a monetary contribution and wished to share personal insights about myself where appropriate because I did not want to leave the women without giving something of myself in return. Reciprocity for Baca Zinn (1979) during her research meant "spending time with informant family members, listening to their concerns, providing assistance when asked or helping their children with their school work" (p. 216).

\section{Meaalofa/Koha}

To minimise any financial cost to the women taking part in the interviews, I offered transport to and from a venue if the interview was not conducted in the participant's homes. When I contacted the women for the second phase interview, they were reassured that having their babies at the interview was not only acceptable but in fact welcomed as it provided me with 
an opportunity to meet them and also removed any concerns the women may have felt about finding childcare. Light snacks and beverages were provided at every interview and at its conclusion, as a gesture of appreciation and thanks (see above), I offered each woman a supermarket voucher. One woman declined to accept the voucher as she said she was financially secure and asked that it be given to another woman taking part in the study who may be in more financial need than herself. After a couple of minutes of discussion, I suggested that the woman herself could offer the voucher to a recipient in her church community who may benefit more from it, to which she agreed.

\section{Research relationship (power relationship)}

It was important to minimise any differences that may have arisen in a hierarchal relationship between me and the women. Philo (2003) describes this difference as a "gulf" - which can sometimes be "massive" or sometimes more "nuanced" - in terms of "attributes, identities and background" (p. 8) between the researcher and the researched. Some of the differences between myself and the women contributed to the power differential in the relationship, for example: age (I was 17 years older than the eldest woman who participated in the research); employment (I was employed by the university in a research unit); geography (I lived 5 minutes drive from the central city whereas all the women live in Porirua City which was approximately 15-20 minutes drive from the central city); educational levels (of the four women involved, 2 finished their formal schooling to the end of secondary school, 2 completed courses); formality (I presented them with formal consent and information forms); access to hi-tech equipment (I arrived at the interviews fully equipped with the latest interviewing technology); access to personal and confidential information (I required information from them for this research study whilst not being required to divulge anything personal about myself); and finally, as the mother of two adults and one child, I may have been perceived as being an "experienced" mother i.e. more knowledgeable about pregnancy and motherhood.

There are several ways of dealing with the researcher's privilege within the multidimensional power relationship between researcher and the researched, one of which might be to consider ways to give back to the researched. I reassured the women that my prime interest was in their experiences as described from their own viewpoint, in their own words, constructed in a way that makes sense to them. At the beginning of each interview I explained to them that some of the topics they would be discussing could conjure up some experiences that might be 
emotional for them. Again, they were reassured that they could stop the interview at any time if they felt uncomfortable. During some of the interviews some women became emotional and cried when describing their experience of post-birth. I felt that, as an older woman, I had the maturity to be comfortable in that situation and, most importantly, to stay silent to allow the women to feel at ease with their emotions rather than immediately asking if they were okay or needed anything. I was secure enough to sit quietly until the women composed themselves and were ready to continue. So, in this instance, rather than my age creating a distance, I believe it created a stronger connection with the women. Sharing some of my own experiences as a young mother contributed to breaking down the earlier power relationship where I had access to personal information about the women and they had no personal information about me.

\section{Cultural protocol}

There are several current research guidelines that specifically aim to assist researchers working with Pacific peoples in New Zealand, for example (Health Research Council of New Zealand, 2005). The entire research process, in essence, was guided by the broad protocols that reflect the foundations in fa'aSamoa (the Samoan way) principles (Ngan-Woo, 1985; Tupuola, 1998); ava (respect), fa'aaloalo (reverence) and alofa (love, compassion and concern). The extent to which traditional fa'aSamoa protocols would be observed with the women was difficult as I could not initially gauge the level of significance fa'aSamoa had in their lives. It was a challenge as I wanted to respect those who wished to participate in fa'aSamoa e.g. starting the meeting with a prayer. At the same time, I did not want to impose a tradition on the women if that tradition held little meaning or relevance for those concerned, particularly as Tupuola (1998) acknowledged that not all those "of Samoan descent in New Zealand live in accordance with the lifestyle and expectations of traditional Samoan cultural and social norms" (p. 76). Mindful of this, I ensured that each woman was aware that she could guide the level, if any, of fa'aSamoa protocols for the interview. None of the women asked to start the interview with prayer.

\section{Recording the interviews}

The information sheets the women read before the interviews stated that the interviews would be recorded either by audio-tape or digital and approval was needed as part of the criteria to participate. The women were then asked to sign a consent form that again reiterated the interview would be recorded; signing of the consent form was given as formal permission for 
the recording. All the women agreed. The interview would not have proceeded if any of the women declined to be recorded. Consent to record the interview was part of the criteria as this was a salient point of this research - capturing the language and words used by the women themselves; this gave depth and meaning to their stories. I believe I would have been unable to capture fully the essential nature of each interview had I relied on written notes alone. Furthermore, I would not have been able to give my full attention to the interviews had I been distracted by taking notes. Verbatim quotes were also an important tool to validate the themes that emerged. All the interviews were transcribed verbatim by me using the given pseudonym for each woman to identify the audio-tapes and the transcribed interviews (for example Elegi (1)) and any other identifying features such as names or places were altered for reasons of anonymity. The audiotapes and transcripts were stored in a secure location in a locked filing cabinet accessible only by me. The digital and audio recordings, stored on my computer hard drive, will be kept for a period of three years and then destroyed.

\section{Returning the transcripts to the women}

In accordance with feminist research that privilege empowerment and "ownership", each woman was given a copy of her transcript. The desire to share the transcripts with the women was partly driven by the wish to empower the women in the research process but also, importantly, to demonstrate the transparency of the research process. Forbat and Henderson (2005) believe "the practice of showing participants transcripts might be extremely fruitful in achieving research objectives, such as engaging participants to reflect further on their first interview. It is also often used to check the details of or add to their initial conversations" (p. 119). In returning the transcripts to the women I was aware that the women may have had a reaction of surprise when reading their conversations with me.

For all of the women, being interviewed for a research study was a new experience and reading the transcripts could have been unpleasant or even confrontational (Forbat \& Henderson, 2005). For these and security reasons, it was important that I delivered the transcripts personally to each woman rather than rely on the post or email which could have been intercepted by someone else. Personal delivery not only gave me an opportunity to visit the women again but also to reassure them that embarrassment, surprise, or shock at reading their own transcripts was very common. I reiterated the confidentiality of their interviews and reminded them that they had the opportunity to change anything they had said in the transcript. None of the women chose to edit their transcripts. All were encouraged to keep 
their transcripts in a safe location as they contained private information. It was also suggested that they might want to view their transcript as part of a memoir for either their children when they were older or as a recorded account of how they felt during their first pregnancy and all welcomed this suggestion.

\section{Final meeting with women}

I had not intended to meet with the women again however, after the second interview, one of the women commented that she had enjoyed the interviews so much that she would like to meet again. This prompted me to ask the other women if they too were also interested in meeting up again, to which they all responded positively. This would have been a great opportunity to show the women a draft of the themes that had emerged and also to "validate" the data in that "what is discovered may be verified by going back to the world under study and examining the extent to which the emergent analysis fits the phenomenon and works to explain what has been observed" (Patton, 2002, p. 67). Unfortunately, this further meeting did not eventuate as one woman had left the country and another was heavily pregnant. However, a follow-up meeting should be considered a priority in future research of this kind.

\section{Data analysis - Grounded Theory}

The women who participated in my research openly shared their insights, experiences and knowledge during their last months of pregnancy and after the birth of their child. Many times they offered unsolicited and honest descriptions of their situations and some of the factors they felt affected their health and wellbeing during each particular phase. Consequently, I gathered a wealth of stories to be explored. Grounded theory (GT) developed by Glaser \& Strauss "focuses on the process of generating theory rather than a particular theoretical content" (Patton, 2002, p. 125). Charmaz (2000) states that the surge of opinion of some researchers who argue that grounded theory is very similar to positivism (for example an assumption of an objective, external reality, a neutral observer who discovers data, and reductionist) is counter claimed because the position of grounded theory "moves into postpositivism because they also propose giving voice to their respondents, representing them as accurately as possible, discovering and acknowledging how respondents' views of reality conflict with their own, and recognising art as well as science in the analytic product and process" (Charmaz, 2000, p. 510 ). To this end, the analysis of the interview data was conducted through a variation of grounded theory and included the typed transcripts and notes. The transcripts were read several times and entered into NVivo 8.0, a qualitative 
research software package that allows the researcher to consolidate data into manageable thematic chunks. It is acknowledged that some researchers may see qualitative software packages such as NVivo 8.0 as being most useful and beneficial for larger amounts of transcripts (30 or more). However, I contend that the eight transcripts, which were long and detailed and where a multiple of themes emerged, were made manageable by the thematic analysis features of NVivo 8.0 which allowed ideas and topics to emerge from the data. Coding of the data involved a three-step process; open coding (identifying properties), axial coding (relationships between concepts) and selective coding (theoretical sampling). The technique is intended to open up the data: break it down and look for empirical indicators of concepts (Sarantakos, 2005). Text obtained from the literature review and written transcripts generated initiation of high level "concepts" which were split into clear categories to maintain confidence that all relevant concepts generated by the women were included (Sarantakos, 2005).

Each transcript was read several times in order to code data into small chunks to identify indicators of "factors" affecting the wellbeing of the women ("open coding'). Data was positioned within a multilayer classification in which the data dictated how many indicators/factors were included at each level. For example, the women might be asked about the concept of "support" during pregnancy. They might mention "family as important" support; a follow up question might be "what about family is important?" The response might be "because my partner helps around the house". Therefore, a schema may look like: (concept) support; (category) family; (indicator/factor) "partner helps around the house". At this point consideration was given to the data to dictate how many indicators would be included at each level. Identification of common experiences from direct quotes located emerging patterns, all the while ensuring that this process maintained the essence of the women's stories.

\section{Ethical practice}

This research was conducted and informed by a feminist framework and also drew from Pacific research guidelines to ensure it was practised ethically - Guidelines on Pacific Health Research (Health Research Council of New Zealand, 2005). From this standpoint, it was important to me as a researcher that all the women were fully aware of their rights and that the decision to take part in the project was entirely their choice. 


\section{Reflexivity}

One of the defining concepts rooted in feminist research is reflexivity i.e. being self reflective in terms of self-questioning and self-understanding (Patton, 2002, Ackerly \& True, 2007) and constantly being aware of "what I know" and "how I know it". It also helps in thinking about the research process in more depth (Smith, 1999) while acknowledging the impossibility of value-free and objective research. For example, as mentioned above, I was fully aware of the subtle, and not so subtle, differences between myself, a much older, university educated researcher, and the women who were much younger and how that age difference may influence some of the information they would be prepared to share with me. Guba and Lincoln (2000) recommend keeping a "reflexive" journal on a daily basis, or as needed in order to "record a variety of information about self (hence the term "reflexive") and method" (p. 327). Specifically for this research project, I was encouraged to start a journal to record my thoughts regarding any challenges or perceived triumphs during the entire process. I found that although keeping a journal was a new concept it enabled me to crystallise the research process and provided written accounts of the rationale behind the various methodological decisions made for the duration of the project.

The journal (alongside writing this thesis) was a valuable tool that assisted me with writing in the first person, a challenge after writing in the third person (positivist and passive) for my undergraduate degree in Psychology. Adopting an "active" voice was initially a challenge for me, a challenge according to Patton (2002) that involves finding a voice that should engage the reader through rich description, and thoughtful sequencing so that the reader joins the inquirer in the search for meaning" (pg 65). Writing in an active voice places me "within" the research rather than "observing" the research process.

\section{Acknowledgement of Mentors}

Research guidance over and above that provided by my main supervisor was crucial during the research process as it provided me with both personal and additional professional support. Engaging with two researchers from different world views (one a New Zealand-born Samoan and the other a New Zealand-born papalagi ${ }^{6}$ ) provided complementary support and added invaluable insights into the practicalities of carrying out social science research.

\footnotetext{
${ }^{6}$ Definition of Papalagi (shortened version palagi) meaning: a foreigner (Pratt 1892); European, white man (Milner 1966) (Pratt 1892; Milner 1966, as cited in Asiasiga, 2007, p. 8).
} 


\section{SUMMARY}

In this chapter I have outlined Crotty's four-element model i.e. epistemology, theoretical perspective, methodology and methods. Within these constructs I have discussed in detail the justification for the research approach of feminist phenomenological social constructionist research in this thesis and provided a rationale for the use of individual interviews. In the following Chapters Four, Five and Six the research findings are presented. The findings that emerged from the data produced some interesting and surprising insights into how Samoan women view pregnancy, childbirth and motherhood. 


\section{CHAPTER FOUR: PREGNANCY}

It has been the biggest change that I've ever been through, ever, but it has made me ... it's been the most happiest thing to ever happen to me, it's been the best thing to happen. (Geraldine)

Pregnancy, considered by many to be a "transitional" period (Crouch \& Manderson, 1993; Oakley, 1979), can greatly influence what happens up to a year post birth, with the last trimester particularly being a time saturated with decisions, changes, adaptations and forward planning. The planning is for an event; and although mothers-to-be have some idea of what is going to happen, they are never really fully prepared for the actuality of events. For the women in this study, pregnancy was a time of turmoil, happiness, sadness, illness and change and brought many challenges to the participants and their close family and friends. The women mediated between systems (Samoan/New Zealand, health, Christian values and beliefs) in order to maintain an optimistic sense of their wellbeing - whether it was perceived or actual.

This chapter presents the women's own articulation of their "cultural positions" and highlights the differing levels of awareness of the Samoan culture amongst the women, particularly with regard to knowledge around the traditional beliefs. This leads to the issues around their knowledge of their bodies and contraception use. Discussion about the challenges posed by an unplanned pregnancy highlights the difficulty of informing others of the pregnancy due to fear of negative judgement. Both formal and informal support systems are considered e.g. midwives, antenatal classes. Key factors that contributed to the women's health and wellbeing during their pregnancy included a reliable and strong support network of family and friends, financial independence and a sense of confidence of their social positioning. Before progressing on to the findings of the women's experiences of pregnancy, it is appropriate at this stage to introduce the cultural positions and identities of the women as defined by themselves, and some of their perceived traditional beliefs around pregnancy.

\section{Cultural positions of the women: "I am who I am"}

The permeation of cultural traditions between the generations was discussed and it was interesting to note how overt or covert cultural traditions influenced the women's pregnancy 
experiences. It was important to explore where they positioned themselves in relation to their parents' cultural and traditional values and beliefs rather than where others positioned them. In telling their stories, one of the authors of "Being "Afakasi" described having "two different sides" to her identity and the difficulties she faced with not having a place to really "be with that", which consequently left her with profound feelings of "existential loss which underpins my being" (Berking, Fatialofa, Lupe, \& Skipps-Patterson, 2007, p. 58). These feelings of loss, of not quite belonging, of being pulled between two worlds, the risk of being faithful to one meant the relinquishing of the other appear in much of the literature around cultural identity (Anae, 2002; Southwick, 2001; Tiatia, 1998; Tupuola, 1998).

The struggle with this cultural liminality did not surface in any of the women's interviews an important point as I argue this is what strengthened their ability to be confident in "two worlds", their ability to be Sophisticated Mediators. They were confident with their New Zealand-born Samoan identities, the strength of their self-defined cultural positions may in fact have been due to being marginalised (i.e. New Zealand Samoan women exposed to, and with access to Samoan and New Zealand systems). Geraldine expressed that "balancing" two cultures appeared to be problematic for her parents' generation which eventually let go of many Samoan practices. Therefore, it was significant to listen to their own current situations and perspectives without placing any preconceived notions or ideas that stemmed from the literature of where they should be positioned.

One of the themes to emerge from this data was the perceived influence the Samoan culture had on the women's everyday lives, the level of Samoan language competency, knowledge about Samoan culture and the extent of "living Samoan". The women articulated the cultural benefits of being Samoan was the importance placed upon the family and undeniable support. All the women felt the concept and meaning of "family" was more entrenched within their Samoan cultural value system which set them apart from non-Samoan families. Even though they did not consider themselves limited by the culture, they chose to accept this part of their inheritance:

One thing that differs between like Polynesian families and maybe families that aren't is family and no matter how culturally oriented you are, you'll find that family always comes back into it and that's what I found, even though I'm not fa'aSamoa

\footnotetext{
${ }^{7}$ Samoan term used to describe a person of Samoan and Papalagi (Caucasian) descent.
} 
or anything like that, I still value my family and that's really important... it's not necessarily maybe a cultural thing, maybe there's aspects of it that come into it but I just find that we tend to be more closer with our families and we need that support. (Geraldine)

This is an example of sophisticated mediation: while Geraldine denies that she practises fa'aSamoa, she is aware of and fully accepts the salience of the family element which is so embedded within Samoan culture.

The importance of maintaining the Samoan language in New Zealand as part of retaining a strong Samoan cultural identity has been well documented and defended. Hunkin-Tuiletufuga argues language and a strong connection with the culture together with achievement in the educational system will result in what it is to be "truly successful as New Zealand Samoans" (2001, p. 201). In order to maintain this bond to the culture, Hunkin-Tuiletufuga asserts it is better for Samoan language speakers to live in close proximity to each other in order to socialise frequently with each other, particularly in church communities and voluntary organisations, thus, he argues to strengthen the language proficiency.

That said, it has been conceded that while some may "choose" to discard their language and culture, many prefer to accept some parts and not others (Macpherson, 1984 as cited in Hunkin-Tuiletufuga, 2001, p. 201). As pointed out, the opportunity for the women to learn the language was dependent on their various environments. Macpherson (as cited in HunkinTuiletufuga, 2001) states three environments can result in different orientations to Samoan language and culture:

Environment 1: primary orientation is to Samoan values and institutions Papalagi values are overlaid on Samoan values rather than displacing them;

Environment 2: Samoan culture exists alongside a non-Samoan culture and children move between the two ... - they share a common belief that they are in some way Samoan and that it is a valued identity;

Environment 3: Life is oriented to and dominated by non-Samoan language, values and activities and personnel ... in which the parents systematically promote non-Samoan culture ... and limit involvement with or commitment to Samoan values and institutions (pp. 201-202).

It was not my intention to match the experiences of the women to Macpherson's different environments, but rather to provide a rationale of what some believe contributes to the loss of 
language for Samoan people living in New Zealand. The level of the participants' Samoan proficiency ranged from one woman possessing no Samoan language skills at all, two women understanding Samoan but unable to converse in it, to another being a fluent speaker. This reflects the level of exposure to, and use of, Samoan at home as well as the women's own attitudes to learning Samoan in the dominant English-language culture i.e. the level to which they wished to assimilate in New Zealand by preferring to speak only English. Although all the women in the study were New Zealand-born, Geraldine and Henrietta were the only two who reiterated that they were born in New Zealand as though to justify being brought up primarily in a non-traditional way, i.e. not learning to speak Samoan:

I'm New Zealand-born and Mum brought us up here in New Zealand... Mum hasn't chucked any of the traditional things onto me... Mum's been like that since we were growing up. (Henrietta)

I admit I'm not culturally brought up strong in a Samoan kind of society and stuff like that. (Geraldine)

Not being brought up in the "Samoan kind of society" or not experiencing some of the more "traditional things" did not equate to any of the women losing, or feeling they had lost, any of their sense of being Samoan. For Geraldine, her negligible Samoan language skills were not from lack of wanting to learn:

My dad understands Samoan, my mother understands Raro but neither of them speak it... we tried to move over there (back to Rarotonga) when I was younger and we tried to learn the language and stuff like that and couldn't pick it up... got teased and the same thing happened to my mother... so that's where the whole language barrier comes from and why we don't speak it. (Geraldine)

Furthermore, it was not until Geraldine, as a new member of her partner's large Tokelauan family, witnessed the whole family conversing in their "mother" language that she felt a tinge of sadness that she did not know her own Samoan language, not so much for herself but rather as something that she was able to gift to her own children:

I see my partner's family who are still very much into it (culture) and the kids can understand (the language)... I don't understand anything... for me it's quite hard and for my (unborn) son as well, there's really not much I have to pass on to him, culturally, because I'm just not that, do you know what I mean, so that's a hard bit. (Geraldine) 
While Geraldine accepted her own level of cultural awareness, "I'm just not that", she concedes the reality that her own children will not benefit from the perceived cultural depth that her partners' nieces and nephews enjoyed due to being bi-lingual in Tokelauan and English.

Henrietta grew up in a household where both parents spoke to her in Samoan yet she and her siblings opted to respond in English. Although this created frustration for her mother, it was a trade-off Henrietta felt she needed to make in order to avoid confusion with learning the English language:

Mum and dad are both fluent ... they both speak to us in Samoan but we speak back in English... I remember when we were younger Mum used to always tell us off for always trying to speak English... to be honest, I don't think it really helps when you're a New Zealand-born and you go to school and everyday you can hear English, you know, and then you go back home. (Henrietta)

Henrietta's husband is bi-lingual and her attempts to converse with him in Samoan often led to his affectionate jibes about her lack of language skills. Undeterred, Henrietta did not believe that her lack of proficiency in spoken Samoan would pose problems because she could understand it well and should they wish to pass it on to their children, her husband was fluent. Geraldine and Henrietta's experience regarding not acquiring their parents' mother tongue is not uncommon; merely being exposed to a language does not guarantee that the language will be learnt or understood. Nevertheless, in hindsight, both women would have preferred to have obtained a greater level of proficiency in Samoan although they believed their lack of proficiency in speaking or comprehending Samoan did not define them or limit their identity as Samoan.

Geraldine explained how she believed her family evolved into being not that "culturally strong" and how her family created their own cultural identity:

My Dad lived in Samoa when he was really little ... because there was so much influence, not so much German influence but European influence on his family ... I think maybe then they kind of let go of being Samoan, it was more like being halfcaste... I think that kind of diversion in that kind of way that it wasn't pushed on him to be cultural... so his family wasn't very cultural either... (Geraldine) 
This was an interesting insight, i.e. the consequence of living a European way of life or living as "half-caste ${ }^{8} "$ meant her family came to "let go" of being Samoan and relinquished parts of their culture in favour of Western or European culture. I have emphasized parts because throughout Geraldine's interview there were segments that strongly illustrated "Samoan culture" reflected in her own practices and beliefs - thus revealing areas where Geraldine herself negotiated between traditional Samoan culture and Western culture, e.g. accepting what she considered to be a traditional Samoan massage when it was offered to her by her mother-in-law. Geraldine did not view the shift away from "Samoan culture" as being unique to her family and felt that other families with European heritage or names also "stepped back" from their Samoan culture.

Both Geraldine and Henrietta described their family histories from their own perspectives in order to contextualise their current situations. The narratives of the women and their positioning of themselves in relation to their parents' cultural history and traditional beliefs are important in defining how they view themselves as New Zealand-born Samoans. All the women are young and, as cultural positioning is fluid, it is highly likely that their cultural positions will change and adapt in the future as they undergo new life experiences, particularly motherhood. The limitations of this study do not allow me to delve into the depths of "letting go" of one's culture or to explore its meaning.

\section{Traditional pregnancy beliefs}

The women were asked to share any pregnancy beliefs acquired from their families which they considered to be specifically Samoan in origin. Henrietta self identified as being "ignorant" of traditional beliefs. The three others were able to cite some traditional prohibitions during pregnancy such as not cutting or dying your hair; not eating particular foods or not being alone at night. Their descriptions of traditional beliefs were very sketchy and they could not provide the rationale as to why these beliefs were considered important in Samoan culture. It should be noted that none of the women gave much weight to these beliefs which were unanimously described as "superstitious", a scepticism that is confirmed by another New Zealand study (Abel, et al., 2001).

I've heard of some... I've heard some things from distant, like older family members that have said don't do this, don't do that... the hair thing but I don't pay much attention to what they say sometimes... they always kind of gave their 10 cents and I

\footnotetext{
${ }^{8}$ Samoan term used to describe a person of Samoan and Papalagi (Caucasian) descent.
} 
kind of never took it on because I never saw the relevance in it but I haven't heard all that much and just hear things like cutting your hair. (Geraldine)

None of the participants' mothers had discussed these traditional beliefs, perhaps because they themselves were unfamiliar with them, and any information gleaned had come from other older family members. The lack of information was not confined to traditional beliefs, as neither knowledge about the body nor sexuality was openly discussed.

\section{Knowledge about their bodies}

Christianity is found to have a strong impact on the way in which Samoan women view sexual identity/sexuality (Asiasiga, 1994; Tupuola, 1998). Considering the high number of Samoans who affiliate with a religious denomination, it is not surprising the permeation of the conservative religious teachings influencing how women construct ideas about their bodies. However, with this in mind the women articulated they were informed about the basic mechanisms of how the body works, i.e. the female reproductive system, understanding menstrual cycles, the effects of hormones on the body and various other changes brought about by pregnancy. This information was learned mainly through formal schooling in New Zealand ${ }^{9}$ and this point was specifically mentioned by one woman who praised her school for having a really good health and wellbeing class.

Pacific Islanders tend not to ask lots of questions directly related to themselves, or their bodies, particularly with their own mothers:

One of my aunties that I'm close to mainly talked to her about it, yeah but not my mum. (Alma)

Because I think ... not many women in our culture, I don't know, are familiar with their bodies but they tend to think that they know everything but they don't ... but yeah, I think it's good that everyone does know about their body ... I think I didn't really ... I tend to watch movies ... (Elegi)

The women talked about how they learnt about the body in general, usually from friends or close relatives such as aunts, but very rarely was it discussed with parents unless you were "really, really close" to them. One woman said she felt that her parents were more receptive

\footnotetext{
${ }^{9}$ Sexuality education is one of the key learning areas for New Zealand students from years 1-13 (primary to secondary school exclusively i.e. 5 years old to approximately 18 years old). The New Zealand Curriculum is the official policy relating to teaching and learning outcomes in all New Zealand schools. All schools must incorporate sexuality education as part of the school curriculum, schools must consult with their communities when developing health and sexuality education programmes, hence there may be slight differences in the delivery of the learning programmes.
} 
to discussing personal issues about bodily functions, sex and their own experiences once she became pregnant.

Your parents start to treat you differently as well because you're older and so they seem to be a bit more open to your questions and in answering those questions that involve sex. (Henrietta)

Parents feeling comfortable enough to talk about sex after the women found out they were pregnant has a slight irony to it in that discussions to prevent pregnancy were too late. Yet, for many Pacific Island people the subject of sex is still considered tapu (taboo) inhibiting discussion between parents and children (Anae Fuamatu N., Lima, Mariner, Park, \& SuaaliiSauni, 2000; Asiasiga, 1994; Fa'asalele Tanuvasa, 1999). This is not surprising in view of the strong hold Christianity has on Pacific worldviews; this has been deconstructed in feminist literature (Griffen, 1983; M. Stewart \& Hunt, 2004).

Vanessa Griffen in writing her second manual, Caring For Ourselves: A health handbook for Pacific women, attempted to demystify the knowledge women had at the time about their bodies and how they work. In addition she aimed to provide comprehensive information that would be freely accessible to the women, in the context of Christian circumscription and traditional cultural conservatism (Teaiwa, 2010), and to "enable women to have more control over their bodies and their health" (Griffen, 1983). The holistic viewpoint Griffen offers illustrate the factors associated with health such as education, social and economic.

Knowledge of the body in the context of the sexual reproductive system, menstrual cycles, and various other changes the women experienced before becoming pregnant and during pregnancy. All the women pointed to the lack of communication with their parents, particularly, mothers in discussing anything to do with sexual health or about the workings of their bodies.

\section{Contraceptive Use}

Contraceptive knowledge and use among the women was very low, which was not surprising considering the very low uptake of contraceptives among young Pacific women, for reasons as stated earlier which include strong Christian beliefs, preference for abstinence, sideeffects, or lack of information about services (Abel, et al., 2001; Asiasiga, 1994; Fa'asalele Tanuvasa, 1999). The women were not asked specifically about their knowledge of the types of contraceptives available or what method they had been using. However, all indicated that 
they were aware of, or using, either abstinence or the contraceptive Pill. Whilst not a reliable indicator of their overall contraceptive knowledge, this suggests the contraceptive Pill is the most recognised form of contraception. Natural family planning options were not mentioned. Of the four women, Henrietta was the only one actively using a form of contraception before she conceived and consciously planned her pregnancy. The terms "consciously" and "planned" are synonymous with being in control of fertility and thus making choices that lead to becoming pregnant. Henrietta, along with her husband, announced her desire to become pregnant, and actively sought to become pregnant by ceasing the use of contraceptives and vaccinating herself against rubella.

Underlying the cognitive reasons for planning or not planning pregnancy, are assumptions of heteronormativity which refers to the "myriad ways in which heterosexuality is produced as a natural, unproblematic, taken-for-granted, ordinary phenomenon" (Kitzinger, 2005, p. 478). Heteronormativity, combined with religious beliefs and the high value of children in Samoan culture may be a further explanation for the lack of contraceptive use among the women. The influence of culture and religion create the heteronormative conditions by which it is considered the norm for a man and woman to engage in pro-creational sex; therefore, contraception is understood as contrary to social and cultural norms as seen in the example from Elegi who attributes her pregnancy as an act of God:

I think it's a miracle, to be honest I never thought I could have children, so it's really weird for me to be pregnant ... it is like a miracle, 'cause I mean like that's what God wants you to do like this must be the right path cause God makes things happen so I think I've taken a right step in my life, it's probably the best step of my life. (Elegi)

Elegi's example illustrates the way in which she chooses from her spiritual beliefs in order to make sense of her situation. Elegi was quite concerned about her family's reaction to her unplanned pregnancy and articulated the pregnancy as "the will of God". She conveyed that her family, particularly her mother, was especially religious. In her announcing her pregnancy as the work of God she dismissed what could be perceived as a negative event by her family to an unchallengeable occurrence. This interpretation of the event was accepted by her family rather than perceiving Elegi as failing in her obligation not to embarrass the family or avoid the pregnancy. She was not held personally responsible for the unplanned pregnancy - the pregnancy was accepted unconditionally without any retribution from her family - a positive outcome for Elegi and a factor in successfully maintaining her resilience. 


\section{Planned or unplanned pregnancy}

A pregnancy that is unplanned may carry negative connotations of being a mistake due to an individual's lack of responsibility or carelessness in not using contraceptives, or not using them properly, or intentional. Three of the four women talked about their pregnancies being unplanned for various reasons but significantly they did not plan or take precautions not to become pregnant, that is to use contraception or natural family planning to avoid a pregnancy, a conscious decision that put them at high risk. As pointed out earlier, Christianity had a major impact or influence on the women. To avoid becoming pregnant requires a certain level of commitment; if a woman lacked a "firm commitment" she will not use contraceptives consistently or is likely to engage in unprotected sex (Stevens-Simon, et al., 2001) as was the case for Geraldine and her partner. Similarly, another study comparing women who intend to become pregnant with those who do not intend to become pregnant also found a third category of women who were "okay either way" about becoming pregnant/not pregnant, which fell in between the first two categories (McQuillan, et al., 2010). There may be no other reason for putting oneself at risk for pregnancy other than there is no reason not to do so:

I just went into it thinking I was superwoman... to be quite honest we were quite, not into abstinence and stuff but it was kind of like one of those things we were going to wait... we went to a party that night and got really intoxicated and then one thing led to another. (Geraldine)

Geraldine's perception of herself as being "superwoman," i.e. strong enough to control her sexual urges and thus avoid pregnancy, was undermined once she was under the influence of alcohol. Geraldine may have been intoxicated and may have lacked a firm commitment not to get pregnant or been buoyed by her supposed "superwoman" status. However, while both she and her partner both decided to have sexual intercourse, it seems the ultimate responsibility for using contraception lay with Geraldine even though her partner had an equal responsibility in the outcome of possible parenthood.

Henrietta and her husband, who already had two other children from his previous relationship, were keen to start a family; regardless of other health issues Geraldine had to contend with, she fell pregnant a lot easier than she envisaged:

We decided in May, that's when I went off the pill, and then it happened in July apparently... it just happened and now we're just going through the motions of the whole pregnancy thing. (Henrietta) 
In stark contrast to the other three women Henrietta, along with her husband, had meticulously planned their pregnancy and ensured her health problems were sorted out. She describes "going through the motions" of her pregnancy which illustrate that, for her, the thinking and reflection around pregnancy had occurred long before the actual conception took place.

All the women articulated they intended to have children eventually; still, despite not using contraception the unplanned pregnancies, appeared to have come as quite a surprise to three of them. Although they were not actively using any form of contraception and they articulated that the pregnancy was "unexpected", they wanted to highlight that it was very much a wanted pregnancy and all stressed that not going through with the pregnancy (for example, undergoing an abortion) was strictly out of the question and not an option they would ever consider due to their personal, family and cultural beliefs, which for Elegi was explicitly religious. The women considered their beliefs, accepted the values of their cultural and religious foundation; while rejecting other options. The women were adamant that the pregnancy was an earlier than expected occurrence rather than "a mistake" and therefore mistimed would be a more fitting term. This is a heteronormative expectation of eventually having children and creating a family. The self-confidence evidenced in all the women's determination to carry through with their pregnancies would be tested in the months ahead.

It was unexpected ... but I don't really believe in abortion so I thought no, I'm going to keep it. (Elegi)

I wouldn't call him a mistake, I suppose he just came before we were ready... both of us come from families where abortion is just not, you know, accepted, no way ... so it was pretty much we're having this baby so that's it ... so it was kind of like, I think that's why I cried more knowing that if this baby does survive to 13 weeks we're ready... it's a lifetime thing and I can't just go and get an abortion... so yeah. (Geraldine)

The women themselves wanted to maintain full responsibility for what they had created. Not being open to other options was heavily influenced by their own belief systems. Coming to terms with the pregnancy themselves was marred by the realisation that sooner or later they would have to tell others. 


\section{Breaking the news}

The women were keen to take responsibility for their pregnancies but all were daunted by the task of informing their parents and families. How they mediated their situations illustrates the level of skills to either maintain or obtain resilience. The reaction to news of their pregnancies played a major part in how the women proceeded to construct their pregnancy experiences and expectations. The undeniable fear of telling parents had a major impact on how they positioned or braced themselves when they revealed their pregnancy.

What was that whole telling your parents stuff, what do you mean?

For me 'cause we've been brought up quite religiously, I mean I'm not following anything at the moment but my parents are quite strong in their religion as well ... that kind of meant that it would be a disappointment for them... you know, as it would anyone who is not expecting it and who was brought up in this way ... I didn't want to upset them because this is something I had to follow through on, it meant that there was no going back. (Geraldine)

Parental disappointment and family dissatisfaction with an unplanned pregnancy were common concerns for three of the women, with the potential to alter the existing relationships with their parents. Geraldine's mother's response to the news, early in the pregnancy, was that despite her disappointment that Geraldine was so young, she vowed to support her through the pregnancy and motherhood. Geraldine, expecting "much worse", was very emotional:

At first mum said to me I am disappointed 'cause you're so young, you're only 20 and at the same time you know I'll support you through your pregnancy, she gave me a big hug. (Geraldine)

All the mothers/parents offered their unconditional support, some making decisions that had an immediate impact on the women's current situation:

I didn't want to tell her but I told her straight away... like she was real, real shocked so she made me come back home, she booked me on the next flight home the next day. (Elegi)

Rather than oppose her mother booking a flight back to New Zealand, Elegi felt it was the right decision and accepted this unreservedly in spite of feeling embarrassed at having to give her immediate resignation to her full-time job. 
Despite being married and thus conforming to cultural expectations, Alma was still anxious about announcing her pregnancy to her family. As Donnelly (1992) points out: "the belief that a married woman who is having a baby will be pleased to be pregnant is commonly held from a Samoan and obstetric perspective" (p. 258). Whilst not "unhappy" to be pregnant, Alma was convinced her family's reaction to her pregnancy would be negative because it was an unplanned event. As is fairly common for many Samoan families ${ }^{10}$, Alma and her husband lived with her large extended family in one house and contributed to the expenses of that household and therefore the pregnancy would affect the extended family (Statistics New Zealand, 2010). Alma delayed sharing the news with her family until she was about four months pregnant - long enough for both her and her husband to come to terms with the situation but also time to plan ahead.

Anxiety about telling family members about the pregnancy influenced how, when and where they were informed, with most women concerned about selecting the "right time". The time lapses between confirmation and informing others gave some women the opportunity to get used to the idea themselves and gain more confidence. Geraldine was two months pregnant and although she had already confided in her mother, tension developed between them because Geraldine delayed informing her father.

I was like oh gosh dad's going to kill me, even though I was 20, I didn't know how he'd react, I know that had I been 14 I would have been dead. (Geraldine)

Eventually she approached her sports-mad father during a time when his being distracted worked in her favour:

Thank goodness the rugby was on and the game was going really well... I just started bawling my eyes out and he said 'oh my gosh, what's wrong?' and I was like 'Dad, I'm pregnant' and he goes 'What? Really?' And he just went quiet and then we started talking about the game and he was commenting on the game and I think maybe that was to kind of deflect him from being overwhelmingly angry, but he was fine... my mother's reaction was worse than what my father's was. (Geraldine)

\footnotetext{
10 In 2001, 30 percent of Samoan people were living in extended family situations - down from 36 percent in 1996. By comparison, 29 percent of the Pacific population and 8 percent of the New Zealand population were living in extended families in 2001.
} 
Geraldine's approach to tell her father was carefully considered and mulled over for some time as the timing was absolutely crucial. She planned for two possible outcomes - negative or positive - by informing her workplace, making appointments with family planning and organising a midwife, she therefore felt she was prepared should her father ask her to leave the family home. If the outcome was positive, which it was, she could demonstrate to her father that she was taking full responsibility for the pregnancy by planning ahead. Geraldine's experience highlights how she could maintain her resilience by planning for two outcomes.

The women's misgivings and anxiety about sharing their pregnancy news was unfounded, with all being supported by their parents and family. Telling others the news was a significant turning point in the pregnancy; and the difficulty the women faced with this issue should not be underestimated. Their expectations of how others would react heavily influenced how they approached the situation and how they viewed their future.

\section{Sources of support}

The women's stories highlighted support as being a significant part of the wellbeing of the mothers. The offerings of the different sources of support for the women allowed them to pick and choose from each system (for example family versus technology) in a manner that reiterated the strength of their decision-making.

\section{Family}

Sources of support during pregnancy set the tone for the childbirth experience and motherhood and it is well documented that there is a connection between high levels of social support systems and positive wellbeing during the maternity period and shortly thereafter (Oakley, 1992). Advice from well-meaning family or friends was appreciated although, at times, overwhelming. The women coped with this avalanche of information by unravelling the advice or information given before deciding which was most useful for their own situation.

Considering this was the first pregnancy for all the women, they were very confident to reject information they felt was irrelevant - picking and choosing the advice. They were making judgements between the advice and information that was useful while disregarding the rest without offending anyone, particularly close family. This was a challenge but a mediating 
skill all the women eventually mastered. Partners, mothers, mothers-in-law and families provided much of the practical and emotional support during the last trimester of pregnancy, strengthening relationships as the family focussed on delivering a healthy baby.

The support from all the mothers was overwhelming as illustrated by the following examples: Alma's mother had a heart condition and Geraldine's was undergoing cancer treatment during the early and late stages of the women's pregnancies. Alma's mother's heart condition was frequently debilitating, limiting her ability to provide physical support. Alma understood and accepted this situation and was content that her mother provided "other" forms of support. Interestingly, much of the support she nominated as receiving from her mother-in-law was social and physical:

She's (mother-in-law) really good... she will take me places like shopping, movies, and going to the library... a couple of months ago they (parents-in-law) took me out to watch rugby, the All Blacks in town... they take me out to beaches, the Plaza, Te Papa... (she) takes me out to lunch and dinner so I'm like really spoilt by her. (Alma)

Drawing a particular type of support from one source to compensate for its unavailability by the primary source is interesting. Alma's mother could not provide the necessary physical support she required so she utilised someone with similar status, her mother-in-law, to fill this void. Some may view this as opportunistic but it allowed Alma to build on the relationship not only with her mother-in-law but also her family-in-law and partner. It gave her more "connection" with her partner's parents which made Alma feel like she was being treated "like her (mother-in-law's) own daughter".

In contrast, Geraldine, rather than drawing the support she needed elsewhere, instead drew it from her mother when it was available. She was quick to point out that "we weren't both sick; I wasn't sick, I was pregnant”. Geraldine described her mother and herself as being very similar in respect to work ethic - working hard, taking responsibility for everything and working themselves "to the grave" - and they resolved to help each other by ensuring each of them rested.

We pretty much slowed each other down which was good, but she was pretty much my greatest support. (Geraldine) 
They "took a lot of time out for each other" and would do things together to help them achieve their goals. These opportunities not only allowed both of them to provide support to each other but gave them time to develop another side to their relationship. As a result this brought them together, not only strengthening their bond in a way that helped her mother reflect, open and share experiences of her own pregnancy, but also for Geraldine to understand her mother more intimately, something that was very new but welcoming for Geraldine to experience:

I had never known my mum to have any sense of like herself, you know, feelings for herself... and then when she was talking about her pregnancies and how she felt and the support she wished she had, it kind of all made sense... that's a side of my mother I've never seen before which was really good... her advice was more important to me and I took more of it in because of that. (Geraldine)

These two very different examples of strengthening relationships are salient. Pregnancy and the impending addition to the family not only created a newfound closeness in one relationship but it also forged a new connection and bond in another and these were definitely important factors for the women's health and wellbeing. The evolution of the relationships helped to build a solid foundation that would at times be tested and challenged in the postnatal period.

\section{Partners}

Not surprisingly, partners were mentioned as a main source of social, emotional and practical support for three of the four women. It was generally acknowledged that they could not offer advice as, apart from Henrietta's husband, they too were inexperienced. The uniqueness of each partner's support in the form of rubbing their backs, making them breakfast, ensuring they ate well or just knowing the woman's limits was a significant help and ensured that the partners became an intimate part of the pregnancy.

The partners demonstrated a high level of commitment to both the pregnancy and the women, were protective and reassured the women that they could rely on their support. Feeling cared for, loved and part of a secure relationship was vital in contributing to the women's overall emotional wellbeing.

My partner... the fact that he was just there for me and wanted to look after me. (Geraldine) 
Having a lot of support from your partner because we both planned it anyway and so... gosh when he found out he was always looking out for me, it got a bit annoying but it's nice. (Henrietta)

Friends and family members played a crucial role in supporting the women, a role which was a precursor to the support the women would receive throughout their pregnancy and postbirth. While the support was important to the women's experiences, the women carefully mediated between the support that met their own personal, individual needs (for example registering with the right midwife) and support that solidified aspects of the collective needs (for example registering with a midwife who was able to meet their cultural requirements i.e. large contingency of family members at the birth). The formal support also played a large part in the construction of the women's maternity experiences.

\section{Formal Support}

\section{Antenatal care}

The New Zealand government provides free Maternity and Health Care services to New Zealand citizens, women with two year's permanent residency status and women eligible through other criteria under the Eligibility Direction (New Zealand Ministry of Health, 2010c). Women may seek private care with obstetricians and private maternity hospital which may incur charges as do some antenatal or childbirth education classes, or tests at a private laboratory. The provision of free maternity services ensures women and their families have access to care during the antenatal, birthing and postnatal period; up to six weeks post-birth. Pregnant women are required to choose a Lead Maternity Carer (LMC), either a midwife or a practising obstetrician, who is responsible for organising a woman's maternity care. They may provide all the woman's care or share the care with one, or more, other practitioners and also provide information to assist with decision-making during pregnancy, preparation of the birth and for parenting" (New Zealand Ministry of Health, 2010c). Most women and their families (over 75\%) choose a midwife for this role (New Zealand College of Midwives Incorporated, 2010). The local District Health Boards provide voluntary fully-funded antenatal classes for both first time parents and those who have attended the classes.

\section{Looking for a Midwife}

All the women were given information about midwives and the role they play in pregnancy and most were advised to engage a midwife when their pregnancies were confirmed. Despite 
a study examining antenatal care attendance by mothers of Pacific infants which showed that Samoan women living in New Zealand were more likely to access antenatal care very late in their pregnancy (Low, et al., 2005), all the women in this study began looking for a suitable midwife either immediately or very soon after their pregnancies were confirmed. Reasons for this difference may be the women in this study took the advice of family members to seek antenatal care early because it fit in with their own belief system of seeking early care; accessing the health system early for a positive outcome. The women were all New Zealandborn, they did not have another system they could access that would provide the care they required, choosing to access a midwife early illustrated they believed in the New Zealand health system to provide them with the maternity care.

Engaging a midwife proved to be more difficult, time-consuming and complicated than the women had anticipated despite being given a list of midwives to contact. After spending a significant amount of time phoning and emailing a midwife provider who subsequently supplied incorrect contact information, Henrietta contacted the midwife provider once again to try and resolve the problem. Eventually, feeling frustrated, she went back to her GP:

I emailed them back (midwife provider) and said "Look, this number doesn't exist. Can you please give me the correct number?" and didn't hear back from them so I scrapped the Wellington one, I scrapped the one down in Porirua City and I ended up going back to my GP. (Henrietta)

Henrietta was proactive and conducted an internet search for midwives in her local area although this too led to further misinformation. This hit and miss process ended up frustrating the women and made them feel as if their eventual association with a midwife was not of their choice but rather a function of scarcity in the market:

When I met my midwife it was pretty much the end of the road kind of thing because I ended up having to go through the hospital. (Geraldine)

I ended up just going with them (midwife)... I ended up with them "cause I hadn't heard back from the (other) ones. (Henrietta)

Although these two examples of securing a midwife by chance rather than by choice were unsatisfactory, they are not uncommon, as borne out by findings from the 2007 Maternity Services Consumer Satisfaction Survey (Dwyer, 2009). This ad hoc process eventually had a positive impact for some of the women. 


\section{Midwife Characteristics}

Asked whether there was anything in particular they were looking for in a midwife the women cited finding a midwife in the first instance as paramount, followed equally by convenient locality, "nice" personality and appropriate qualifications. Initially, ethnicity was unimportant to the women, which is supported by a study which found Pacific Island women did not prefer a doctor of the same ethnicity to herself (Ekeroma \& Harillal, 2003). Considering the Samoan community is relatively small, reasons for the preference of a doctor from another ethnic community might be due to confidentiality issues, trust issues and/or privacy concerns the women may have e.g. a practising doctor from the Samoan community may know the family or members of the woman's extended family intimately.

The Samoan midwife Alma eventually registered with proved to be invaluable because she was able to speak Samoan to Alma's mother who had very limited English, thus ensuring her mother was well included in the pregnancy development and was well versed in the pregnancy process and therefore better prepared to support her daughter. Language ability proved to be an important factor in this case, even though it was not initially considered a priority for engaging a suitable midwife. Mediating between what was necessary for Alma as an individual and what was necessary collectively for her mother, played a significant role in Alma's decision to remain with the Samoan midwife - another example of sophisticated mediating.

A Samoan midwife did not initially sit well with Geraldine because at their first meeting the midwife, upon learning Geraldine's surname, immediately started speaking to her in Samoan. The assumption that Geraldine could speak Samoan immediately offended her and the offence was exacerbated when the midwife questioned her:

She's like 'Oh, how can you not understand me?' and I was like 'Well, we're not all like brought up that way... what are you expecting me to be like?'... I did kind of feel like oh shivers I think I might need to go with someone else. (Geraldine)

Geraldine persevered with the relationship, due to the lack of alternatives more than anything else, and eventually they arrived at a mutual understanding and respect:

I just have to take her the way she is and it's vice versa with her as well. (Geraldine) 
Geraldine's example illustrates the importance of working together with a midwife and creating that opportunity to do so. In view of Geraldine considering registering "with someone else" they were able to forge on and work out boundaries and expectations of each other which resulted in mutual respect and the continuation of the relationship, an example of mediating at an individual level in which the outcome was not ideal but Geraldine chose to view it as a positive alternative.

One of the major assumptions the women made was they would be registered to one midwife but in fact they were required to register with a "team". Henrietta, was adamant she wanted one midwife only and although she felt compromised she decided to view it positively.

I wanted one (midwife), just the one, because I just wanted to have that one-on-one relationship with my midwife... in the end I thought oh, in a way it's probably good you get expertise on all four of them. (Henrietta)

She took stock of the situation and decided it was advantageous to work with four midwives with a diverse range of expertise and experience. The difficulty of finding a midwife is problematic for many New Zealand women, not just New Zealand-born Samoan women (Dwyer, 2009; Television New Zealand, 2008). The current midwife system failed the women in this study in that it took away their power to choose a midwife to suit their particular needs; however, although they were subjected to an ad hoc process of securing a midwife, the women, who could not change the system, changed how they viewed their experience into a positive one.

While the women themselves illustrated the skills of successfully mediating between systems, it should be noted there are examples of midwives mediating between two systems. Traditional midwives are considered mediating agents between their indigenous knowledge (passed down through generations) and the colonial health system (Barclay, et al., 2005; Hattori, 2006; Macpherson \& Macpherson, 1990). Since the early 1970s, most Samoan fa'atosaga $^{11}$ have undertaken short training programmes in an attempt to improve knowledge and skills - in conjunction with their traditional beliefs and practices this has meant contemporary belief and practice is an amalgamation of indigenous and introduced elements

\footnotetext{
${ }^{11}$ Fa'atosaga is a person who is regarded as a specialist (midwife) and a professional by herself and her community. TBA (traditional birth attendants) is the term used to refer to midwives and other women who have attended the Department of Health training course (Barclay, et al., 2005)
} 
(Macpherson \& Macpherson, 1990, p. 184). The pattera (indigenous midwives of Guam) who practised traditional midwifery were labelled by the colonial military as "dirty old women" (p. 10) and their methods were considered to be "ignorant superstitions" (Hattori, 2006 , p. 10). In the bid for colonial and medical control over the women of Guam, the Americans attempted to replace the pattera knowledge of midwifery by offering native-nurse programmes (Western knowledge base) within the American based hospital. However, this backfired and the graduated nurses "did not simply replace traditional methods with navy teachings, but instead syncretised newfound knowledge and techniques to create a hybridised practice of maternal care" (Hattori, 2006, p. 18), an example of sophisticated mediation of taking the best of two worlds to utilise the best outcome for the women they were serving.

\section{Antenatal classes}

Although offered to all, there were many reasons why the women attended or did not attend antenatal classes; of the three women who booked for antenatal classes, one did not attend, one was currently attending; while the other was yet to attend.

Supported by their midwives and families, the two women who did not attend classes were confident they would get the information they needed elsewhere. Geraldine's decision not to attend classes created some concern for her partner who felt that they would miss out on important information. Her mother, however, was confident that Geraldine's family's support was equally beneficial.

Mum was like you don't always need to go to antenatal classes, sometimes you can just wing it... as long as you have your family and a good support system. (Geraldine)

Another fervent reason for not attending antenatal classes was the dread of being in the same room as other pregnant women:

I couldn't stand them (other pregnant women), I was the only pregnant woman and that's it... so that's why I didn't want to go to antenatal, I just did not want to be around other pregnant women. (Geraldine)

Henrietta, on the other hand, was keen to start antenatal classes as soon as possible; she found them particularly informative as they dispelled some stereotypical notions of birthing and 
provided her with extra options she may not have otherwise considered:

They gave us information at our first antenatal class and the different positions you can have baby that was interesting... I thought oh, you don't have to just have it on your back, yeah, I didn't even know you could stand, I've seen on the hands and knees, yeah, it was very interesting, there are so many different positions... it's just whatever you're comfortable with. (Henrietta)

All the women considered antenatal classes to be one of many avenues to acquire information, rather than the only option. While not everyone was enthusiastic about attending, Henrietta found them to be very informative and they contributed to her desire to stay well organised during the maternal period. As part of the formal support, technology played a major part in connecting the women to services that were accessible from the comfort of their own home.

\section{Healthline}

Healthline is a national toll-free 24-hour telephone general health service, funded by the Ministry of Health. Calls are monitored by registered nurses with tele-nursing training who assess health needs and provide information and advice to assist callers about the best level of care. Although this is not the only telephone advice service available, it was the only one specifically mentioned by the women in this study:

Healthline is 24 hours and I had heard of Healthline through my other friend... it sometimes happens at night and that's when I ring 0800 (Healthline), everyone will be sleeping and I don't want to disturb them... (Henrietta)

Henrietta talked about Healthline as an invaluable form of support, particularly during the night or when she needed "more clinical" advice, and she continued to utilise it post-birth. All the women and their partners were employed during the pregnancy period, this served as support also.

\section{Paid Employment}

The women articulated employment and employers as a source of support. All the women were in paid employment at the time they conceived and three of the women's partners were in full time, permanent employment. The partners' employment gave the women a sense of future financial security and the option to resign from their employment, if necessary, an 
option utilised by Alma who ceased working at a restaurant as the smell of food was intolerable in the early stages of her pregnancy.

The Parental leave ${ }^{12}$ entitlement was accessed by Henrietta and Geraldine ${ }^{13}$ only. The financial cost of supporting children was taken seriously by all the women, as illustrated by the need to plan and organise their monetary situation including savings and future earnings. All the women were inspired by long-term financial gain, personal independence and a continuation of their financial self-sufficiency.

The three and half months, I'm going to apply for the three and a half months which is that paid parental leave and then I'll just see from there how it's going, 'cause I might just go part time depending ... it kind of works well with my husband and I 'cause he does night shift so if I do go back to work straight after that then I will look after baby in the evenings and he goes to work and does the dayshift and I will go and work ... it works out well for both of us but we will see how it goes closer to the three and a half months. (Henrietta)

During their employment, employers' were considerate toward the women if they were unwell due to morning sickness or unable to carry on their duties due to physical strain. Although unsure whether or not they would return to work, three of the women officially applied for maternity leave intending to finalise the decision after the baby was born.

\section{SUMMARY}

Pregnancy as the transition to childbirth and motherhood conjured up many changes for the women. They were frank and honest in their discussions, naming the difficulties they were experiencing and how they mediated their way through this period. Even before the pregnancy was confirmed the women had to navigate their way through the multitude of advice that was given or sought and decisions that had to be made. Successfully mediating between these new events and experiences provided the women with a solid foundation which would serve as a key factor for their health and wellbeing during their journey into motherhood.

\footnotetext{
${ }^{12}$ The Parental Leave and Employment Protection Act 1987 (New Zealand Government, 2008) prescribes parental leave entitlements for female and male employees and protects the rights of employees during pregnancy and parental leave. The Parental Leave and Employment Protection (Paid Parental leave) Amendment Act 2002 and the 2004 amendment provide eligible employees with some parental leave payments of up to 14 weeks, available through the Inland Revenue Department. ${ }^{13}$ Geraldine applied for unpaid maternity leave for one year but did not return to her original place of employment.
} 
The women were adamant their self-defined cultural positions were important in being secure with their identity - acknowledging their being Samoan in New Zealand neither places them exclusively within either culture which places them in a place of strength rather than deficit (i.e. ease with which to access both systems).

Contraception use among three women was non-existent which led to pregnancies that although unplanned were very much wanted. However, although three of the pregnancies were mistimed, this did not lessen the fear three of the women had in sharing their good news with others particularly parents - the family disappointment at the lack of planning may have contributed to this fear, as being married did not make bearing the news any easier.

The pregnancy period was a time of changes - physically, emotionally, spiritually, but it was also a time to strengthen existing relationships and also forging new relationships, particularly with those that were providing support namely family and friends. Unlike what other authors found, the women of this study initiated antenatal care early in their pregnancies, although not all the women took advantage of the services available to them, namely antenatal classes. The midwifery system of registering with a team of midwives did not suit the needs of the women, who preferred the one-to-one relationship with one midwife.

The women's experiences were, at best, positive i.e. pregnancy and parenthood were considered the best thing to happen to them and, at worst, not the end of the world and bearable. The women were able to mediate successfully between cultural and health systems to help them make sense of their pregnancy and impending childbirth and motherhood. 


\section{CHAPTER FIVE: CHILDBIRTH}

The women in this study articulated mixed emotions about their childbirth experiences. Negative and positive feelings can coexist and, further, support the multi-dimensional character of the birthing experience which is influenced by both physical and psychological factors (Waldenstrom, Borg, Olsson, Skold, \& Wall, 1996).

The expectation and experience of labour and childbirth were crucial foci for this research. The expectations drew out many of the women's hopes and fears: much of the hope was around being able to deal competently with the labour itself while the fears were around the health of the unborn child. As first time mothers, these were totally unknown areas and therefore, exploring these aspects provided an opportunity to investigate how the women prepared for childbirth, and the factors that contributed to their childbirth experience. A key factor was the ages of the women in this study at the time they gave birth - Alma, Elegi and Geraldine 20 years old; while Henrietta was 29 years old. Communication and planning was a theme that was identified as important, not only between the women, their families and the midwives but also between the midwives and the delivery suite.

\section{Preparation for the Birth}

A formal birth plan ensures that the woman's wishes are clearly documented so that any maternity professionals involved with her care are aware of her preferences (Families, 2010). By the first interview, all four women had either completed their birth plans or were about to meet with their midwives to complete them. The birth plan was seen as an organic guideline in which flexibility to modify the plan up to, and even during, the labouring process, was critical. This gave the women an even greater sense of control over their situation but, it should be noted, that for the health and safety of both the baby and the mother, all the women were willing to relinquish their written instructions should complications arise. In addition, as commonly found none of the women in this study were overly worried if their labour did not progress according to the birth plan (Whitford \& Hillan, 1998).

With this in mind, all the women found the birth plan to be beneficial as it allowed the women to feel that they were well planned and organised. The point the women made about being prepared for the unexpected suggests the women's own sense of control over the 
birthing arrangements remain intact and further supports the need for the birth plan to be adaptable. Whatever the actuality, the women acknowledged the possibility of several outcomes and were willing to adapt their birth plan as circumstances dictated.

The mismatch between the birth plan and the actual experience, as occurred with three of the four women, could have had dire consequences with regard to their emotional wellbeing if they had viewed the birth plan as rigid and something that had to be adhered to, no matter what eventuated. The women hoped their own personal conduct during labour and the birth itself would differ from the stories they had heard of other women's experiences or the images they had seen on television and film.

(During labour) I'm probably yelling out and screaming and my facial expressions ... I don't want them (students) to see that ... when you see it on TV like the lady is hard out screaming and yelling ... I'm a bit nervous for that part ... I just hope I'll be looking good when I'm pushing out baby, I just don't want people to see the ugly me, well that's what I'm worried about. (Alma)

Elegi referred to her midwife in terms of the role she would play during childbirth and the trust she placed in her, as she perceived her midwife to be far more knowledgeable about her body than she herself was, handing over complete control to her midwife:

I'm going to make sure I'm going to listen to the midwife 'cause she knows what's best and what I should do, yeah, I'm just going to listen to her so I do everything right. (Elegi)

Interpretation of what constituted a good childbirth experience was influenced by what the women had read in the media, books, antenatal classes or discussion of other women's experiences. The women's concept of natural childbirth was articulated as the safe delivery of a live birth which emphasised little or no drug intervention, a reasonable length of time and minimal interference from the medical profession. The emphasis on non-intervention childbirth may be seen as romantic (Crouch \& Manderson, 1993), however, the women articulated this as a pragmatic aim.

So what that entails (birth plan) is no drugs, hopefully, but I'm a wuss and then at the last minute I'll probably ask for it, or try for something. (Geraldine)

I want to go natural, you know, that's what I say but I mean if the need arises I want to make myself prepared for medical intervention ... just hearing from other 
people's expectations, some people get quite disappointed when they don't follow through and I don't want to be like that, I don't want to be stressing out for that reason ... trying to get a different sort of mindset. (Henrietta)

Maintaining a positive mindset regardless of what eventuated during childbirth was important for the women to embrace as this would help to buffer extreme disappointment. Using the information from others' childbirth expectation experiences was a way to prepare for what is an unpredictable situation. However, what the women could control was the birthing venue. One of the most important aspects the women did not compromise was the location where they would give birth. Since the confirmation of their pregnancies the women were adamant of the choice of birth venue.

\section{First signs of labour}

A homebirth was not considered an option by any of the women, all of whom said they expected and preferred a hospital birth. The main reason for this was the knowledge that medical experts and professionals would be on hand with all the necessary equipment and medication, which reflected a high trust in the medical profession and the hospital's support system:

A hospital birth because I don't really want to have baby at home, I want to have it at hospital where I can have people who know what they're doing around me. (Elegi)

The women felt empowered knowing they were surrounded by medical professionals who they perceived as being able to control and manage the birth. This was part of the women's own "loci of control 14"; in choosing a hospital birth they believed it was better to lose control in a hospital rather than in their own home.

A "show" 15 " or a contraction was identified by the women as the first sign of impending labour which in all cases initiated a phone call to the midwife or the delivery suite. The contact with their midwife was mainly for encouragement and reassurance that their labour

\footnotetext{
${ }^{14}$ Locus of Control refers the extent to which individuals believe that they can control events affecting them. Individuals, who have a high internal locus of control or referred to as "internals", believe that the events in their lives are primarily the result of their own behaviour and actions. Individuals who have a high external locus of control or referred to as "external", on the other hand believe that the events in their lives and organizations are primarily determined by chance, fate or other people (Vaughan \& Hogg, 2002).

${ }^{15}$ Bloody show: A small discharge of blood mixed with mucus from the vagina is usually a clue that labour is about to start. The bloody show may appear as early as 72 hours before contractions start (Merck, Sharp, \& Dohme Corporation, 2010).
} 
was progressing well. During the early stages of labour all the women were in their own homes with their mother, partners, and father and extended family members. Alma needed her midwife to make a home visit to provide some direction and leadership:

I wanted the midwife to come and check me, my main one at my home 'cause I wasn't too sure what was happening... I just didn't know what to do, didn't know whether to stay home and wait for baby to come out or listen to the midwife and go straight to there (delivery suite) but then they'd send me straight back but then I was just so confused. (Alma)

Alma's lack of success in persuading her midwife to visit (calling and requesting a visit several times) contributed to her feeling powerless but angry as she felt uncertain about what was happening. During her time labouring at home Alma received a "Samoan fofo" (traditional massage) given to her by her mother-in-law which relieved the pain but she continued to feel confused at the lack of support by her midwife. Alma struggled with the decision making, i.e. should she stay home or go to the hospital, which was due to the conflicting information she received from her midwife and the delivery suite. Alma was sent back home from the hospital twice which undermined her confidence and made her anxious.

Henrietta's midwife did make a home visit but not until she was almost ready to go to the hospital. During her early labouring, Henrietta was satisfied with the regular communication and subtlety of her midwife's assessment:

She (the midwife) had spoken to me through the day as well, so she would have got a sense of whether I was close listening to my voice, that's what they told me beforehand as well, if they listen to you and they can sort of hear what you're sounding like. (Henrietta)

The intuitive approach of the midwife gave Henrietta added reassurance in her midwife's ability to assess her situation from afar. While this may not suit all women in all situations, and not all midwives have the experience and capability to do so, it enabled Henrietta to stay at home as long as she could as communication with her midwife was frequent. Staying home was important for Henrietta as it sustained her sense of independence and control over her labour which she may not have experienced if she went to the hospital early. 
Elegi had a home visit by her midwife during her early labour and while she knew her contractions were "normal", her uncertainty about the intensity of her contractions increased her concern about her progress:

I didn't know what was going on and I didn't know if it was normal... I didn't know if it (contractions) meant that I was going to have baby now. (Elegi)

These examples illustrate the differences in the midwives' approaches to the women, the impact this had on them, and the importance of good communication in their approach to early labour. While the method taken by Henrietta's midwife resulted in her being calm and relaxed labouring at home, there were other factors too that helped: Henrietta's preference to be at home for as long as she could; prior knowledge of how the midwife would assess her situation; and the regular communication between them. For Alma this may not have necessarily been suitable and may not have had the same outcome as other variables were at play, such as Henrietta's independent personality and her particularly relaxed approach to childbirth.

The women felt that communication between the midwife and the delivery suite should be more consistent to ensure that they themselves did not make unnecessary trips to the delivery suite only to be sent home several times. For Alma, having to communicate with the delivery suite herself as well as with her midwife, whilst striving to concentrate on her contractions transformed her mood from unconfident and anxious to angry and very frustrated:

Well, the midwife, I rang her and she said that I had to go straight to delivery suite but then they kept saying something different so they kept sending me home and so I rang my midwife and told her what's happening and she said oh just wait for a while and if it gets worse then ring the delivery suite ... (Alma)

Unfortunately for Alma, her situation was further aggravated by the reluctance of her midwife to make a home visit and this further strained the relationship between them so that it eventually ceased on a less than happy note. All the women were comfortable in their homes during the early stages of labour although Alma and Elegi were concerned they would give birth at home, due to their vagueness of whether the first sign of labour meant immediate birth. This highlights the lack of information the women received regarding the different stages of labour; how women can identify these stages, how long each stage would take and how long the labour could likely take was a major concern as was a lack of physical 
examination or physical sighting by the midwives during labour. This was a key factor that may contribute to women being able to be more relaxed in the home.

\section{The role of the midwife during labour}

The attending midwife for the labour was not necessarily the midwife the women had during their antenatal care as the women were registered with a team of midwives. During labour the role of the midwife, as perceived by each of the women differed. Clinical expertise and guidance was something all the women assumed would be provided but ideas on how closely the midwife would monitor or intervene during labour differed. It was important to Henrietta and Geraldine for their midwife to be as unobtrusive as possible:

We didn't really need her (the midwife) until the last minute and I think that's what they try to do so it's more natural... through the contractions everyone was pretty much around me rubbing backs... and when it was time she thought I was ready to push, she was pretty much there... she left it up to me until pretty much the last minute. (Henrietta)

Geraldine was firm in her belief in the role of the midwife:

I think because I had all that support as well it just wasn't a big deal, they (midwives) were kind of just there to assist me and that's it. (Geraldine)

For both Henrietta and Geraldine the role of their midwives was clear - assisting with the birth with very little or minimal intrusion. The midwives maintained a distance that enabled the women to retain a level of autonomy over their labours and this, in turn, created a space for the families to play a more intimate, practical and personal role during childbirth which elevated the experience to another level. This theoretical midwife "space" was opened up by the women's belief and stance of the midwives' position - assisting. The role switch that saw the families play a significant role in the "space" is an illustration of the strength mediating can create towards positive outcomes.

Although less reliance on the midwife was appropriate for Henrietta and Geraldine whose births were relatively absent of any foreseen and actual complications. Yet for Alma and Elegi, whose labours and births were peppered with difficulties, closer monitoring of and during the birth was necessary. 
There was arguably no doubt that how the women perceived the midwives (i.e. negatively or positively), had a major influence on their experiences. All the women eventually accepted the consequences of registering with a team of midwives meant no guarantee that their preferred midwife would attend the birth; however, the impact of this was greater than one woman anticipated.

\section{The role of the midwife during birth}

The lack of specificity in the four women's expectations of midwives during childbirth indicates an assumption that the midwives would feature prominently and therefore, was not something they felt needed reiterating. Geraldine was the only one of the women who was registered under the hospital maternity system and therefore her antenatal appointments and her delivery were attended to by the on-duty midwives rather than a small team of external midwives.

Midwives were obviously significant during childbirth, however, the impact of their presence and the level of satisfaction expressed by the women about them varied. For Alma, the importance of continuity of care by the same midwife was imperative, not only because she had established a trusting relationship with her midwife but so had her mother. The "main" midwife spoke fluent Samoan and was therefore able to converse with Alma's mother throughout the pregnancy process and they looked forward to this relationship continuing through to delivery. However, Alma's "main midwife" was unavailable for the birth which meant Alma was further stressed by the necessity of acting as a translator:

I preferred the Samoan lady to explain to my mum what was happening rather than me have to explain and I can't really tell cause sometimes my mum will ask, you know, what is she (Pākehā midwife) saying ... but I couldn't explain myself cause I was in so much pain. (Alma)

Geraldine had no qualms about her unknown attending midwife:

I knew that I would get people (midwife) that I didn't know so it wasn't really like a big deal for me to having a stranger basically talking me through it so I wasn't too worried. (Geraldine)

The contrasting examples demonstrate very different attitudes towards their attending midwives. For Alma, the absence of her midwife not only affected her but the support 
network that Alma relied on, i.e. her mother. Geraldine expected to have a midwife she was not familiar with, and therefore was not disappointed. However, Geraldine considered the role of the midwife minimal rather than playing a major role in her birth.

\section{The role of the family during birth}

For Samoan families birth is a family affair, everyone is included and relatives are encouraged to attend (Kinloch, 1985). The women aware of this tradition embraced it and fully expected family and extended family members to attend the birth. However, the women maintained their authority over who would attend the actual birth. It was clear that the presence of their mothers and mothers-in-law at the birth was considered to be nonnegotiable. However, for Henrietta this had to be discussed with her husband as his initial reluctance to have her mother present was based on past bad experiences with his previous mother-in-law. Eventually he agreed after he realised how important it was for Henrietta:

He was the one who decided ... 'cause my mum would have automatically thought she's in there (at the birth), I think a lot of Pacific Island mothers think they're automatically in there ... for me it was automatic, anyway. (Henrietta)

All the women expressed that the major challenge of birth, for them, was its unpredictable nature and therefore having their families in attendance to support them translated into a sense of confidence to face the unknown - they were aware that their personal vulnerability would be strengthened by the presence of their families. In one form or another, facing the childbirth experience with the full support of their families turned the event from a solitary or twosome occasion to a positive family affair. As planned, all the mothers, three out of four mothers-in-law and three of the four partners attended their respective births. Regardless of the time of day or night, extended family members were present either at home or in the hospital waiting rooms. Geraldine illustrated the absolute necessity of family members which was echoed by all the women:

I needed that support I think 'cause I had a birth plan and I had all these things that I wanted to do while I was giving birth and because I couldn't do them, I needed my family around ... it was a ceremony. (Geraldine)

Mothers and aunts played a particularly important role in the birth, particularly for Elegi whose partner was not able to be present at the birth: 
Well, my aunty and my mum, they were like just ... I mean just the little things like getting ice for me, holding my hand, encouraging me to push and stuff like that and that helped me a lot ... very important, I don't think I could have gone through that by myself. (Elegi)

Assistance provided by the families included emotional, physical and spiritual support and all the women mentioned "rubbing my back" or "holding hands", receiving "a massage", or words of encouragement. This was a significant and prominent part of the childbirth process as it demonstrated the occasion was shared by all. Having their support people close by enabled the partners to take a break or to rest, knowing that the women would be well supported by the female members of the family in their absence. The scene was set for the imminent birth once the women were admitted into the hospital and settled into their rooms.

\section{The experience of birthing}

The actual experiences were varied; none experienced a completely natural birth as desired although all had vaginal births. Elegi, Alma and Geraldine reluctantly succumbed to having an epidural, Elegi endured a forceps delivery. All three stated the labour was not quite what they had expected, i.e. the length, the unexpected feelings, the exhaustion and the sheer physicality involved proved to be "far from what I expected".

I so wasn't expecting that (experience of labour) I thought I would have contractions on that day and have baby on that day, but no, it wasn't at all what I was expecting. (Alma)

What I didn't expect was that I would be on my back like the whole time ... so I definitely wasn't prepared for... it was hard 'cause I couldn't push properly. (Geraldine)

While Henrietta expressed a sense of personal achievement and satisfaction in being able to adhere to her plans during labour and maintain a drug-free birth, she decided to ignore her own body signals and felt this was the reason why she suffered a torn perineum:

I teared, I thought I would... you can feel your body saying you don't need to push yet but I did it cause everyone was saying push... I think my family were saying push 'cause the midwife was saying push and I was saying oh okay, I'll push... and then I ended up tearing, but that was all good, baby was fine and as long as he was fine. (Henrietta) 
In Geraldine's case, the supporting role took on a surprise turn for her father. Geraldine did not expect to ask her father to be present at the birth, and earlier had specifically explained that although her relationship with her father was close and she wanted him at the hospital, he would not be in the birthing room. However, the reality was not what she had expected and she became very upset during her labour and asked for her father, a request her mother thought "odd" but supported. Geraldine explained that at the time she felt her parents and her partner had specific roles to play dependent on her relationship with each of them and this was illustrated in her orchestration of their physical positions during the birth:

... I wanted my dad in the room but I wanted him on my side ... my dad is the huggy one and my mum is very strong ... so I kind of wanted her at the battlefront so to speak, but I wanted my daddy by my side ... I had my dad by my side and my partner. (Geraldine)

Geraldine not only demonstrated an acute sense of self-awareness of her needs but also showed a confidence beyond her years (she was 20 years old at the time of her birth) which enabled her to challenge the social and cultural mores of childbirth. She chose to exercise personal autonomy and to ignore society's unwritten laws or values regarding the attendance of males other than medical practitioners or partners, and included her father in the birth experience. Furthermore, as well as knowing what she needed (e.g. nurturing), and who could provide it, she articulated it at a time when perhaps she was at her most vulnerable; a very good example of sophisticated mediating at an individual level. Geraldine's father's presence at the birth was testament to the close relationship they shared and although initially her mother expressed a degree of surprise at the request, it was not contested by anyone else in the birthing room, i.e. Geraldine's partner or midwife. In fact, in support of this request the midwife arranged for a bed sheet to be placed over the lower half of Geraldine's body to maximise her personal privacy. The plea for her father to be present at the birth of his grandchild was highly unusual and relatively rare; a general literature search on grandfathers attending the birth of their grandchildren did not yield any documentation or commentary about the phenomenon, however personal communication revealed this may be an unexplored trend among young Samoan women ${ }^{16}$. This unusual event also highlighted a sentiment expressed by all the women - childbirth was a family affair which may produce exceptional

\footnotetext{
${ }^{16}$ Personal communication with a close Samoan relative of mine revealed that her three New Zealand-born daughters requested their Samoan father to be present at the births of all their children. In the last 8 years, he was present at the birth of three of his grandchildren - at the side of each daughter. Both my relative and her husband are deeply religious and currently practice within their faith.
} 
situations. Geraldine articulated that childbirth was more than a celebration but a "ceremonial" occasion - one which in line with Samoan tradition involves the entire family rather than the more common New Zealand tradition of including the partner, and possibly the mother.

One of the key points to emerge from this data was the significance of family members' presence - in particular mothers, mothers-in-law and, for Geraldine, her father - at the birth of their first child cannot be fully appreciated without the stories from the women themselves. The positive contribution made by the attendance of family members was unquestioned; it created a unique dynamic to the event and far exceeded any of the women's expectations. The attendance added to a shared experience that would strengthen the connection between the families. This was salient particularly if the birth was problematic.

\section{Dealing with a difficult birth}

Alma and Elegi both described difficulties during their labour and birth:

The midwife was trying to get me to push, she didn't want the doctors to come in at all but the doctors came in and took over and said I had been in labour too long and needed to get baby out now. (Elegi)

Eventually a decision was made to prepare Elegi for a caesarean birth, but at the last minute Elegi gave birth assisted by forceps only. However, the lengthy labour Elegi had endured meant she was extremely fatigued by the time her child was born which disappointed her immensely:

Really exhausted to the point where I couldn't even keep my eyes open when she was born ... I expected to spend some time together awake (with my daughter), not falling asleep ... that didn't go to plan. (Elegi)

Similarly, Alma could only sustain consciousness for short periods of time after the birth due to shock rather than fatigue; she described her labour experience as a "really difficult birth" and traumatic. Immediately after giving birth, expecting her baby to emerge kicking and screaming, Alma was met by a baby that "came out, no voices" at which time she fainted. 
The depth of emotion caused by this incident was revealed in her descriptions:

I was so scared I thought he was not alive, usually when they (babies) come out they kick and scream but nah ... 'cause baby came out, he was not alive, he didn't look alive when he came out... everyone was quiet when baby came out... (Alma)

Immediately, her midwife pushed the emergency button to signal for medical help:

Everyone (doctors) just rushed in but I must have conked out or something ... I looked into the corner side and baby was there and they (doctors) were trying to wake him up and they did the breather thing on him... and they took him straight to intensive care... I was really scared I thought he was not alive. (Alma)

In addition to the chaos of medical staff working to resuscitate her baby, Alma struggled to cope with her own shock and uncertainty, she had to deal with the understandably intense emotional reactions from her mother:

My mum was crying, my mum thought that if something was wrong with baby she will blame the midwife and the delivery suite people cause I was in so much pain ... she asked (told) the midwife if anything is wrong with baby then she's going to blame them. (Alma)

Alma was calmed by her eventual reunion with her newborn baby at intensive care, almost three hours after she gave birth.

The midwife finally came back and the doctor and they said that baby was alright, he was just in shock or something and he was breathing and he's just got a little bruise and then they said it's quite normal for babies to have a bruise ... they're giving him antibiotics just to help with his bruise and they said that he's crying in the room and that made me happy ... and then everyone cried. (Alma)

The doctors' explanation concerning the events leading up to her baby being placed in intensive care was very vague and was intended to normalise, even simplify, what was obviously a very serious and potentially precarious medical situation. The decision to administer antibiotics and unclear explanations made by these medical experts in both Elegi's and Alma's cases was unquestionably accepted by both women. Even though they had very little understanding of the true extent of their situations - a blind and obedient acceptance of the knowledge of the medical experts as being categorically accurate was found in other research findings (Baker, et al., 2005). However, I would argue that for both these women it 
was not completely a "blind and obedient" acceptance that allowed them to move forward from their uncertain situations rather than the reality of producing a healthy, live baby that prevailed over the chaos. Rather, both women chose to concentrate on the positive and best aspects of their experience (for example the baby was alive and healthy) instead of dwelling on the traumatic circumstances of a serious medical event. Choosing the optimistic viewpoint was part of the mediating process that sustained their strength and resilience to deal with the current situation than ruminate about the past, something the women illustrated throughout the interviews.

\section{Communication with medical staff}

The breakdown in relationships between the women and medical staff could have far reaching consequences. The lack of consultation or inclusion of the women during her labour adds to the confusion that can invariably occur during labour:

You know how they go out of the room and then they start talking and then they come back in and they say oh we might do this and we might do that so we spent about 2 hours kind of just waiting, I can't remember exactly why I got induced but I remember the contractions coming on invariably by about $3 \mathrm{pm}$ and by that stage... pretty much from $12 \mathrm{pm}$ to when he was born I was strapped into that little baby monitor thingy and it was just frustrating. (Geraldine)

In addition, the level of communication between the women and their midwives was wideranging; there were instances where two of the women were not happy with the level of communication they received from their midwives, while two women were satisfied. For Alma, however, the relationship with her main midwife deteriorated due to the lack of communication and her absence from Alma's birth (which ended up being quite traumatic) and these were the principal factors in the eventual breakdown of their relationship. This was further exacerbated by the fact that not only did the midwife not visit Alma while she was in hospital but when she finally decided to visit Alma at home a few days after being discharged from hospital, she offered no apology or even an explanation for her absence. Alma was still upset and disappointed with the situation and "didn't want to look at her" and thereafter "couldn't be bothered" with the midwife. Alma embraced traditional values and beliefs to a greater degree than the other women and therefore disregarding the presence of the midwife, who effectively was a guest in her home, was a powerful and deliberate statement. Within a Samoan cultural context this would be interpreted as extreme rudeness, particularly towards an older person. 
At the first interview, Alma was shy and the least confident and most reserved of all the women. Therefore, the behaviour described by her above required a reasonable level of selfconfidence and self-assurance which, while not immediately evident at her first interview (demure, unassuming, and softly spoken) was certainly apparent by her second interview (confident, self-assured, and more talkative). This suggests Alma's transition from woman and daughter to her new status as wife and mother aided the cultivation of her self-confidence and allowed her to stake her position from a place of strength. Alma felt let down by a maternity system her main midwife was part of and therefore, within her own personal belief system, she did not believe she was indebted to her midwife in any way.

This standpoint allowed Alma to mediate between her traditional values (respecting the elders) and her individual position (reclaiming her power) as well as resolving an issue in a way that was appropriate for her - another example of sophisticated mediation at a cultural individual level. The unfortunate breakdown of this relationship could have been avoided earlier with a better method of communication.

\section{SUMMARY}

For all the women, regardless of whether the experience of labour matched their expectations, or whether they experienced difficult or relatively unremarkable births, the outcome of producing a healthy, live baby outweighed any lingering disappointments or frustrations they may have harboured. The women uniformly praised the hospital staff, particularly for the assistance in settling the baby and breastfeeding.

Much literature around childbirth experiences considers the impact negative experiences have on women's emotional wellbeing, as one such author describes here:

Women who lose their own sense of control and experience pain, forceps, drugs, Caesarean birth, long and difficult labours often feel guilty that they have not managed well, or angry that they have not been well prepared. They are left unsure of themselves and their abilities. (Bergum, 1989:127 as cited in Crouch \& Manderson, 1993)

While all the women in this study reported differing levels of satisfaction with the birth experience itself, each of them underwent at least one of the experiences, as described above by Bergum. However, the impact was not akin to the emotional outcome of women according 
to Bergum. On the contrary, the women of this study portrayed very positive attitudes postbirth regardless of whether the birth was described as a negative or positive incident or whether they were satisfied or dissatisfied with events. For example Alma's birth was described as a very traumatic birth experience in which her baby was born "not breathing", although she had no control over the situation this did not have any lasting impact on her post-birth as she was just so grateful her baby survived his ordeal. What emerged from my data was the childbirth experience itself contributed to the women's self-confidence and selfesteem.

As evidenced throughout the women's interviews, a degree of negotiation helped determine what took place during labour and childbirth for example a natural birth. Although these desired outcomes did not always eventuate the satisfaction lay with the baby being delivered safely. That said, the women mediated between the paradigm of "natural" birthing (controlled by the women themselves, an egalitarian relationship with attending practitioners, minimal intervention) and the paradigm of "medical" birthing (medical practitioners are experts, medical and clinical intervention necessary, experiences of childbirth removed from consciousness) (Crouch \& Manderson, 1993, p. 41) accepting both models as a means to aid their own individual process of birthing a healthy, live baby. For example Henrietta and Geraldine had birth experiences that were autonomous with minimal intervention with their midwife as assisting the birth rather than Elegi and Alma who relied on medical "experts" and their advice. Regardless of the different childbirth experiences, the construction of what constituted a "good" birth or a "bad" birth was based on the women's own ideals and aspirations.

The final stage of the transition to motherhood was taking baby home. New motherhood brought just as many challenges for the women as it did joy. 


\section{CHAPTER SIX: EARLY MOTHERHOOD}

This chapter is concerned with the months after giving birth and the women's development and construction of "motherhood". The women in this study were first-time mothers so for each there was a sense of discovery, not only of herself but also in relation to those around her. First time motherhood calls for substantial changes ushered in by a different phase of being. Becoming a mother was about taking on multiple roles in that the woman was suddenly responsible not only for her own changing needs but also the needs of her baby, and the expectations of others. The women demonstrated skill in navigating between these often competing situations.

Being part of the "parent circle" brought many benefits that included being taken seriously as an adult by the women's families. A limited and stereotypical image of motherhood, i.e. "do what mothers do, change nappies, clean the house, give baby a bath", and the belief that women were able to switch to "mother mode" instantaneously added to the wonder and disbelief of actually becoming a mother.

All the women who at pre-birth considered themselves to be well organised, and in control of situations were now faced with the unpredictability and chaos that motherhood brought: having to deal with new routines or none at all; a new status - mother; new surroundings and a new person - which created a new dynamic for their families. However, the women's expectations of motherhood and experiences matched in some parts of their journey, while in some parts were relatively unexpected.

\section{Welcome home}

The homecoming can be quite a celebration. Alma had a large welcoming dinner with extended family and friends and receiving the baby into the family was an important event, one which Alma completely embraced and thoroughly accepted.

Interviewer: Earlier you talked about a welcome dinner, who was there? Alma: I had the in-laws, my partner's family, my 7 brothers were there with their kids and wives and the elders of our church, he was there too he read the bible and stuff ... uncles and aunts, it was real packed it was a good dinner. 
In contrast Elegi went from the hospital to the house of her dying grandmother, with whom Elegi was very close; family and friends had congregated there in large numbers. The elder women of the gathering insisted on passing the baby around in maternal admiration, which made Elegi very nervous:

...like there was a lot of people from church that came and a lot of people were trying to hold Abby and stuff and I kind of really didn't want them to hold Abby... I didn't want anyone else to hold her. (Elegi)

These examples emerged from very different situations yet they both illustrate the importance of involving family in welcoming a baby home. For Samoan women, passing the baby around is a common thing to do, particularly among older women and is particularly poignant at a time when someone is gravely ill, as the baby represents the continuation of life. Elegi, whose emotions were a mix of happiness, sadness and uncertainty, relied on the physical closeness of her daughter to help keep her composed, so it was necessary for her to try and regain control over this uncertain situation. To keep her daughter close to her without offending the older women, she told the women her baby needed to be fed, changed, or to sleep which to some degree was an exaggeration of the truth. This desire to satisfy her own individual needs far outweighed the cultural obligation to the older women - an illustration of successfully mediating between cultural expectations and her individual preference.

For many of the women returning home as a "mother" brought a mix of unexpected emotions.

\section{The early days}

The experience as described by the women was not so different to their expectations in that their anticipation of motherhood was often referred to in terms of transition from a world they once knew and controlled, to a world to which they had to acquaint themselves. The women expected that the early days of motherhood at home with their baby would be full of vague and ambiguous meanings:

Tiring, messy, everything that I don't really like, obviously elements of being disorganised and not being on time, I think I'll be extremely happy, I hope so, I'll have my little boy ... I know my whole world is going to be thrown into the air, so just catch what I can. (Geraldine) 
Geraldine's descriptive prediction of motherhood resonated with the other women. Selecting the challenges they can deal with was a coping mechanism many of the women attempted to adopt.

In reality, regardless of the substantial support they received, in the early days the women also felt "lost" from themselves. This was perceived as a temporary period of confusion, particularly during a time when there were so many simultaneous physical and emotional changes happening:

I think I was just really frustrated because nothing was going to like a schedule and I think for me I expected him (baby) ignorantly to fit in with my schedule as opposed to me fitting in with his and us working together, so I was shocked... and days kind of went past and I didn't know what day it was and I was half asleep most of the time... I was just unorganised and I felt a bit lost in my head... (Geraldine)

The experience of returning home was tinged with nerves at being left alone with the baby. Although the women knew they had the support of the family, the sudden dawn that the baby was physically their sole responsibility and they might do "something wrong" was at the forefront of their concerns. Alma was so nervous of going home she asked "the nurse if she could come home with me".

Returning home was a revelation for the women of how expectations can and do differ from experience:

I was expecting, I don't know, like it wouldn't be as hard as what it was... I thought it would just be bed, bath, feed them, bath them, put them to sleep and play with them and that's all but no, it wasn't that simple. (Elegi)

I expected that he would be a hard baby and he will cry all the time if I put him down but I had to wake him up every 3 hours so I could feed him and checking his nappy, he was a sleepyhead... he was quite quiet so that made it easy for me. (Alma)

The quotes illustrated expectations of the first days at home with the baby that were poles apart. Overall, the early days were a mixture of emotional and physical turmoil coupled with the shock of baby not fitting into a pre-existing routine. The women adopted coping strategies, e.g. sleeping when the baby slept, that allowed them to persevere with the knowledge that their situations were temporary. 


\section{Feeding the baby}

Establishing a successful feeding regime for the baby was one of the most significant elements of the first weeks at home with baby. It is well documented that breastfeeding is the ideal for feeding a new baby because it provides a complete balance of vitamins, minerals and nutrients which help the baby fight infection and aid in optimum physical growth (New Zealand Ministry of Health, 2010a). It is recognised as being highly beneficial for both mother and baby in that it not only provides a foundation for the health of the new infant but it also provides a positive contribution to the health and emotional wellbeing of the mother, as well as economic value to the families (Butler, Williams, Tukuitonga, \& Paterson, 2004; New Zealand Ministry of Health, 2009, 2010a).

In New Zealand breastfeeding is promoted by midwives encouraging women to feed the baby as soon as it is possible after birth. Over the last eight years, breastfeeding rates for Pacific mothers have consistently and steadily decreased (from 61\% in 2002 to 53\% in 2007 for exclusive and full breastfeeding 6-8 weeks). This could be attributed to women returning to the workforce and babies being cared for by others. Pacific women are considered a key target group to help promote breastfeeding in order to increase these rates (New Zealand Ministry of Health, 2009). The public health promotion "breast is best" supports the commitment and determination to provide better services and support for women to breastfeed and was one of the driving forces behind the creation of the National Strategic Plan of Action for Breastfeeding 2008-2012. One of the initiatives from the Plan was to ensure women in New Zealand had support services available either in their local community or somewhere easily accessible.

Of the women in this study, one recorded that she had stopped breastfeeding at two weeks, while three of the women breastfed up to the second interview (up to 12 months post-birth). It should be noted that breastfeeding is influenced by moral, social and cultural contexts but is most importantly a very profound personal and subjective experience. Breastfeeding is popularly described with terms such as "successful" or "unsuccessful"; however, like this thesis other literature refers to breastfeeding as "a 'competency to be attained' rather than something which every woman can do "naturally" (Larsen et al, 2008, as cited in Burns, Schmied, Sheehan, \& Fenwick, 2009). 
In preparation for breastfeeding and to support mothers to breastfeed an increasing number of lactation consultants are now available in the community. During their last trimester of pregnancy two of the women of this study accessed a local Pacific breastfeeding group which provided the services of an experienced Pacific lactation consultant. Although all of the women had observed breastfeeding from either family members or friends, the classes were an invaluable tool in preparation for their own breastfeeding experience:

I used to go to breastfeeding classes... she came into the hospital and stuff and when I was pregnant with her (daughter) I went to breastfeeding classes.... they try to encourage Pacific mums to breastfeed instead of bottlefeed. (Elegi)

I definitely recommend to people having a first baby or even those who may be having their second and they just need a refresher... they were really helpful the breastfeeding courses, I had about two or three courses. (Henrietta)

For the women breastfeeding was considered an extension of their intention for a "natural" maternity experience which assisted the bonding development seen as intrinsically associated with breastfeeding (Crouch \& Manderson, 1993). All the women expressed a keen interest in breastfeeding and did not anticipate any difficulties as they felt well supported, well prepared, well informed and within their families it was "second nature" to be able to provide sustenance to their baby. However, the perceived simplicity of breastfeeding challenged the women, some more than others, as they found it "real hard", demanding, complicated and time-consuming particularly when faced with a crying, hungry baby, when they were in a vulnerable state. While the women felt the classes were a great help, the practicalities of breastfeeding proved to be more than they had anticipated as they had not accounted for the physical and mental exhaustion that beset them after birth.

\section{Establishing breastfeeding}

The establishment of breastfeeding was an expectation and priority by the women encouraged by the midwives. In the first couple of hours after birth: "they put him on me and I gave him a feed." (Henrietta)

The main concern around breastfeeding was the "technique", one of the many skills the women were to learn. In the hours or days after birth the focus was around developing a solid breastfeeding method suitable for each of the women. This concentrated effort, on top of dealing with visitors and the physical and emotional demands post-birth, sometimes left the 
women completely exhausted yet still determined. The women felt the practical support from the nurses was helpful:

I was a bit nervous 'cause I was a bit scared that I might do it wrong... I didn't know how to lie him so it was good that I had my mother in law there and the nurses telling me what to do. (Alma)

Uncertainty about what to do, concern about technique were very common for all the women. Leaving the hospital before breastfeeding was fully established remained a grave concern for all the women who were at various stages of breastfeeding patterns by the time they were discharged. Elegi who spent the longest time in hospital, due to baby being unwell, did not record any difficulties with breastfeeding and was strong in her belief that through "perseverance" all women should be able to breastfeed. However, Geraldine who spent two days in hospital and two weeks at home "persevering" with breastfeeding decided to bottlefeed. Henrietta articulated her disappointment at the minimal time spent in hospital (i.e. 33 hours) and specifically associated this with the initial difficulties she encountered; however, she eventually achieved a solid breastfeeding regime. All the women praised the availability of the Pacific lactation consultant, particularly the home visits, despite their different outcomes, suggesting the value of maintaining this national initiative.

\section{Establishing bottlefeeding}

The decision to end breastfeeding often occurs when a chain of small events converge, leading to a lactation crisis and subsequent weaning (Manhire, Hagan, \& Floyd, 2007). Deciding to cease breastfeeding for whatever reason can situate the women in a state of emotional turmoil or with a profound sense of failure. For Geraldine breastfeeding was a top priority, coming from a family where breastfeeding was a routine part of motherhood and easily achieved by the female members of her family. Personal expectations were high with the belief she would not face any difficulties. This may have actually aggravated an already uncertain situation:

I just kept trying and trying and trying and it just wasn't working so there'd be times where I would just cry and cry and cry and cry which didn't help him (son), it didn't help my breastfeeding and I was constantly anxious about the feeds, like when I would have to feed him and how I was going to feed him. (Geraldine) 
Making a decision to cease breastfeeding was followed by intense justification from Geraldine:

They [midwives] were giving me all the techniques and pretty much the standard do this, this should help... it wasn't so much the technique, I had the technique down I could do it, he did not want to latch on properly, I didn't want him to starve. The breastfeeding, it was so hard, we spent two weeks nonstop trying to get him to feed, he just wouldn't latch on, so he ended up being bottle-fed. (Geraldine)

The women of this study expressed many beliefs about breastfeeding including its physical and emotional demands. Considering the significant influence families had in this area and throughout their maternity experiences, a surprising factor emerged from Geraldine's story:

It's weird because this is probably the first time I had to look outside my family... it's not their fault, they couldn't understand (the difficulty with breastfeeding) cause it had never happened before, so I kind of took like my midwife's advice about not all babies are breastfed these days and that its okay to bottle-feed, and if you can't do it, you can't do it... I started talking to other friends who couldn't breastfeed either and they helped me because they've been through it as well, whereas talking to my family I think it was just ... not pointless, but I was going to get an answer that I didn't want. (Geraldine)

Geraldine mediated the conflicting messages between her family and their beliefs (best to persevere with breastfeeding) and the midwives' advice (bottlefeeding is acceptable) with ease. She clearly decided to "look outside" of her family for support from others to corroborate with her in her intention to cease breastfeeding. This was the first time she had decided this and therefore clearly indicates the importance of this act in order for her to feel okay that what was she was doing was right.

The strong belief of the "naturalness" of breastfeeding resonated with Henrietta's mother:

I asked for breastfeeding help and mum is like 'oh why are you asking, it should be natural', okay thanks for that mum... I'm like come on mum, gosh it is hard for some people. (Henrietta)

A clash of beliefs often occurred between the women and their mothers, which may have been due to cultural or generational gaps. The women were articulate and active in asserting what they believed was beneficial for their situation. That said, this did not appear to 
negatively affect the relationships which suggests an open communication style was encouraged and supported by both the women and their mothers.

This style of parenting appears to have contributed to the women's confidence to step away from the strong beliefs held by the family to construct new beliefs based on their own context. As highlighted in the literature review, mediating is not altogether new for Pacific migrant families who made a decision to migrate to New Zealand to create better opportunities for themselves and their families. Thus, along with their own culture the families were now faced with another culture that required a level of assimilation. Furthermore, in the Islands, Samoans were already drawing from both Samoan and Western knowledge and health systems (Macpherson \& Macpherson, 1990; Norris, Fa'alau, Churchward, Va'ai, \& Arroll, 2006), thus mediating between systems before migrating to New Zealand.

For those who achieved breastfeeding, there was a clear message to their "success":

I know I've got a lot of friends who have babies at the same time and they just gave up (breastfeeding) 'cause they couldn't be bothered trying to latch the baby on, I would say just try, just keep trying and not take the easy way out. (Elegi)

A meta-analysis of women's experience of breastfeeding found that women often claim the behaviour of the baby is the reason for not establishing breastfeeding or latching-on as was the case for Geraldine (Burns, et al., 2009). Elegi thought women who could not breastfeed were lazy. However, perseverance is more complex and complicated and is often fraught with emotional and social judgement. Breastfeeding is seen as an important aspect of motherhood and difficulties often cause the mother feelings of guilt, shame, embarrassment and inadequacies (Burns, et al., 2009). For Geraldine, other than feeling on a "downer" she did not explicitly accept full responsibility suggesting she viewed breastfeeding as an interactive relationship between herself and her baby, both having a part to play. She addressed the breakdown in breastfeeding thus: while she played her part and persevered and mastered the technique, her baby did not play his part and refused to "latch on" resulting in his lack of weight gain:

They (babies) will if you keep persisting for maybe another two months but I didn't want him to starve, so I thought nah and by that stage I think it would have been too difficult if he was on bottle and breast as well as it would just confuse him and he'd probably favour the bottle anyway. (Geraldine) 
A study describing breast feeding experiences of New Zealand mothers found that the inability to breastfeed could be overcome by the woman's persistence, determination, confidence and satisfaction adding individual, cultural and social dimensions influence each mother's breastfeeding experience (Manhire, et al., 2007). As illustrated by the example of Geraldine who did not see signs of improvement, what took over was a determination to provide immediate sustenance to her baby regardless of the method.

Developing a successful feeding regime, whether the women were breastfeeding or bottlefeeding, was fundamental to the women's health and wellbeing in the early post-birth day as was the support and relationships with family and others.

\section{Family support networks}

For the women of this study, family were a solid foundation of support. As stated in the Pregnancy chapter, it is fairly common for generations of Samoan families to be living in one household. Three women were living within an extended family situation when they first confirmed their pregnancies. Traditionally, the expectation that grandparents share in the development and growth of their grandchildren fulfils a cultural requirement that "children get to know their grandparents and, through their grandparents, gain a better understanding of their links with their past" (Ngan-Woo, 1985, p. 15). Families, particularly mothers and mothers-in-law, were vocal in their desire to be part of the upbringing of the baby by expressing their wish to have the baby independent of the women.

All the women were assured they would receive complete and unconditional support from their networks - partners, family and friends particularly friends who were already mothers. They were acutely aware of their ability to pick and choose the forms of support that suited them:

I think I'll be alright plus we've got our support networks, I think I'm lucky 'cause all my siblings are here and mum is here, if we needed help they're just up the road so I'm quite lucky in that way ... it's quite natural just to have them there anyway. (Henrietta)

During the period of early motherhood, support was in abundant supply with all the women reiterating the significant roles families and friends played, often stating they could not have gone through this process without them. 
It was good, they gave me some space cause obviously I hadn't had much rest or anything, so I didn't see them (family) as often but mum and dad would come in (to hospital). (Geraldine)

However, the women could also find the presence of too many people at once, particularly during the first few days post-birth, overwhelming:

You're still adjusting and you want to have some time on your own, it was good to have mum here to help out when I needed a shower... but in the early stages you probably shouldn't have too many people at once. (Henrietta)

Whether it was adjusting to their new situations, or needing time to rest, time alone even for a short period made a difference for the women and their emotional wellbeing. Not only were the women dealing with their own wellbeing but they were conscious of the need to nurture other significant relationships.

\section{Partners}

Three of the women were in stable relationships from the time of conception to early motherhood. Elegi's relationship with her partner (who was living in another country at the time she found out she was pregnant) officially ended after she gave birth to her daughter. For the other three women the practical support provided by their partners included helping with the baby or housework or being a confidante for the women. The women articulated the importance of the presence of their partners and described their support in very glowing terms. Going from being a "couple" to now being a "family" made their relationship closer, but the women were aware of the changing dynamics in the household and how that could place additional pressure on the relationship.

The challenge of maintaining a close relationship, particularly for fairly new relationships, was identified early on after bringing baby home. The importance of maintaining an already established relationship while developing a new one was significant for the women and sometimes previous experience helped with steering this successfully:

You've just got to make time for you and your partner to have quiet time when you can ... where we just go and do something on our own... I know the baby has taken over your life in those first few months but you've still got to remember the relationships... you don't want that to start deteriorating because of the new one in your life, yeah, definitely important. (Henrietta) 
Balancing "two" new relationships was at times difficult for Geraldine who only knew her partner for two months before becoming pregnant:

... combined with having a baby and having my partner move in, it was a huge thing... I mean we spent a lot of time with each other but it was all kind of revolved around (the baby)... it wasn't until later that we started to get to know each other. (Geraldine)

This new living situation had a major impact on Geraldine as the limited physical space and emotional areas of her life had to be negotiated:

I had my partner there, which was difficult to deal with as well... like in my space ... because I was very this is mine and this is yours, that was hard to deal with as well at that time. (Geraldine)

This was a time of major reflection for Geraldine which at one point became a time of crisis where she felt vulnerable and possibly exposed in her new situation. She realised this was an important time, necessary to solidify their position as a couple and then as a family; yet the process was very emotional.

The strength of relationships was a key factor that contributed to the women's own sense of wellbeing in particular relationships. Maintaining these relationships while also needing to have time "alone" was important, negotiating and mediating between these two dichotomies was, at times, very challenging. However, the women were able to prioritise their individual needs while at the same time ensuring the family unit was coping with the transition of life with the new baby. Addressing the importance of maintenance of relationships was indeed a key factor for the women, as was their relationship with their mothers or significant other females such as aunts.

\section{Mothers and daughters}

Transition to motherhood is aided by the mother's mother: she acts as scriptwriter, director, stage manager and producer all in one (Oakley, 1986, as cited in Crouch \& Manderson, 1993, p. 148)

There was no doubting the influence of the relationship between the women and their mothers or significant other female family members such as sister or aunt. The relationships 
evolved throughout the pregnancy and became stronger once the baby was born. The women accorded their mothers important roles not only in their newly acquired roles as grandmothers, but also in the mother-daughter relationship that had evolved and matured to a woman-woman relationship which allowed the women a more negotiated stance, rather than that of the mother-child relationship. The relationship between them was dynamic at so many different levels - social, emotional, physical and practical.

The women talked about many expectations they had of motherhood including the new knowledge they were to acquire for a multitude of new skills for example bathing, feeding and changing baby, and their own self-care. The women were proactive in finding information from a variety of sources from the time they were pregnant to the early months of motherhood; however, the practical support from the mothers of the women was expected to help with, as Alma describes "learning to be a mum":

Knowing how to bath baby and (change his) nappy, probably what baby wants when it cries, how to react when baby is hungry or tired or feel a little sick, so they'll (mother and mother-in-law) probably train me on that, reaction of baby crying. (Alma)

Passing and acquiring information from the mothers is referred to as an "apprenticeship system" where mothers and other older women carefully train daughters in the art of motherhood. It can also be described as "scaffolding" (as cited in Bird, 2004: 52), i.e. when there is intergeneration learning, i.e. with the generation with the greater knowledge supporting the younger generation and over time that support reduces as competency builds in the learner.

If the women felt they were not coping, going to "mum's place" was a safe and familiar refuge which gave them time to refocus or to rest or to have company that did not need to be entertained. The mothers provided a wealth of support which was a great comfort particularly in the first few weeks of being home. The support provided was anything from cooking, cleaning, massaging the baby when sick:

Mum was good, she would just come and hold baby if I needed to go and have a shower or do just a few little things which was really good... she would cook me up food cause she knew I was breastfeeding, she was really good. (Henrietta) 
The ease with which the women relied on their mothers implies the depth and strength of the relationship:

I think it was just kind of natural for me to let my mum kind of take over and help us as well as his mother as well because I think the both of us really were kind of in a shock, oh my gosh we've got a kid... but yeah, so the first couple of months I relied on my mum a lot. (Geraldine)

All the women expressed that the strong desire shown by their families to be involved with the baby extending beyond supporting the women, ensured a connectedness between the families and the new baby. For the women knowing their families "wanted" the baby enhanced their self-confidence to accept help or to be comfortable with asking for support.

Going over to mum's... her wanting us there every day and her saying that she wants baby there and she wants to change baby and feed him... they all want baby over and when I want to go somewhere or do shopping and my partner is not around I will ring one of them (mother, mother-in-law) and they can't wait to have baby over. (Alma)

All the women fully encouraged and engaged in the establishment and maintenance of relationships between both families and the baby:

One night in the weekend he (baby) goes to his grandma's and he spends time with them as well... he'll go by himself, he's got three aunts there as well and they're all kind of like the same age as my sisters, so it's just like another home for him, so that's his second home. (Geraldine)

Relationships with mothers-in-law were also important to maintain and in order to preserve the relationship with both her mother and mother-in-law Alma does not contest the food situation and risk getting into a disagreement; she immediately assesses whether being "right" publicly was more important than sustaining a harmonious connection with her mother and mother-in-law:

When I'm cooking (baby's food) as normal I used to blend it, now I mash it cause he's a little older but they (mother and mother-in-law) say it's better to blend it so it will help him eat it straightaway so he doesn't choke... I just do it my way when I'm at home... and when I'm with them I do it their way. (Alma) 
The women possessed an ability to swiftly evaluate a situation and make a decision that resulted in a positive outcome, Alma's example illustrated this very clearly. Alma mediated between what she felt was best for her child against what her mother and mother-in-law felt was best. Offering textured, coarser food to her baby (Plunket New Zealand, 2010) as recommended by Plunket ${ }^{17}$ was contrasted by the belief of her mother and mother-in-law that it would be harmful for the child. Conceding to their wishes, Alma satisfied them but also preserved her own decisions as a parent (i.e. accepting Plunket's recommendation) when her mother and mother-in-law were not around. This act not only contributed to maintaining the relationship between Alma and her mother and mother-in-law, it also demonstrated to the mothers that they were an integral part of the baby's upbringing and their advice was valued, thus further strengthening the relationship. This said, all the women felt the experience of their mothers to be an invaluable resource they had access to which influenced and added to their own parenting styles.

Although the women demonstrated sophisticated mediation between systems, Alma was the only one who expressly articulated how she utilised both Samoan and New Zealand systems. When she returned home, she buried the placenta in a place that had meaning for her family; she decided to not dress her son in red clothing for fear the child's skin would turn red; at the same time, she accepted parenting recommendations from Plunket. Although Alma stated she does "most things the New Zealand way" she described the traditional medicines offered by her Samoan mother-in-law for her sons' nappy rash and cold:

It was like a green leaf that she mashed up with water, she's got some at home, they said it helps heal the baby's bottom ... yep, it helped, so that's what he has when he gets the rash ... and also he had a blocked nose and coughing phlegm and she made this medicine to help it, it was coconut, sugar cane and this green leaf thing, so I have to eat it and then put it in the cloth and then squeeze the juice out for him to drink... on the spoon, I didn't feel comfortable taking it but I just thought I would give it a try to see if it would help... and it helped as it unblocked his nose. (Alma)

Immediate results reassured Alma in the faith that Samoan medicines are beneficial for her son. However, she still maintained a slight scepticism in its ability to heal:

\footnotetext{
${ }^{17}$ Plunket is the largest provider of support services for the development, health and wellbeing of children. These services are available free to New Zealand families with children from birth to 5 years old.
} 
Sometimes I get worried about the Samoan one 'cause you don't really know what they put in there but I make sure that they explain to me what's in the medicine... so I don't have to worry that ... 'cause sometimes when I see red spots I get worried I'm like oh no, it's that medicine but then I'll get the Plunket come in and they'll say it's alright it's just the heat rash and stuff. (Alma)

Not completely convinced of the healing power of Samoan medicines, Alma was not completely convinced of the New Zealand healing methods either and stated she was only given Pamol by the GP or Plunket as "there wasn't much else they could do". Amalgamation of the Samoan and New Zealand methods appeared to be satisfactory for Alma. While successfully mediating between formal (Plunket) and informal (family) support or Samoan and New Zealand system, other support groups were also central to the women during this time.

\section{'Other' support}

Dependent on their needs at the time, the women selected a variety of support systems that were available to them. They were not averse to stepping outside of their immediate support systems if they felt it was necessary. This was illustrated in the way they accepted or rejected particular types of support.

Community groups are often an important aspect of support for women, but organised support groups - whether social or informational - were not a priority for the women of this study. The value of the advice on breastfeeding or childbirth was appreciated by the women but they discounted any further social involvement with groups such as the Plunket mothers' groups or antenatal groups. The women did not consider any national or local groups for social purposes suggesting the women were well supported in that area.

Technology in the form of cellphones, internet and telephone featured as significant media and ways of maintaining contact with friends, family or other external forms of support. All the women were in their early-late twenties - they were the first generation to grow up with such technology, including internet and cellphones, which were incorporated into their lives. Although statistics show that households with Pacific people were less likely ( 23 percent) to have internet access (Statistics New Zealand, 2004) all the women in this study had access to the internet at home. Henrietta found the internet a major source of support particularly during the months post-birth. Disappointed that she left the hospital with little information 
about her son's congenital physical disability, Henrietta accessed and registered with a well known website, eventually joining to engage with other parents:

Just reading other peoples experiences on that forum, they had been through it all too and yeah, everyone pretty much been through the same thing, well how they feel, it's like ... oh gosh. (Henrietta)

This website was a very important connection for Henrietta as the support was specific to her experience. Receiving support and encouragement from mothers as well as couples, she did not have to leave the house to attend a physical group, and most of the time she received immediate responses she could access at any time. During the interview it was evident from Henrietta's emotional responses that the impact of her baby's disability was profound, including feelings of isolation and feelings of being overwhelmed. The situation eased when Henrietta sought support from people who were in the same situation with a child with the same disability, experiencing similar challenges. She thus built another external support system which she could depend upon, and the specificity of the support enabled her and her husband to get her through some challenging times. For all the women, access to the internet gave them admission to multiple communities and at any time.

For more selective networks the women relied on their mobile phones. Ownership and use of mobile phones for 15-29 year olds in New Zealand is very high (90\%) so it was not surprising that all the women owned their own mobile phones and utilised this as a support tool. The women did not only use their mobile phones to stay, or improve, their “connectedness" with others (Walsh, White, \& Young, 2008), but also as a way of feeling secure if away from home (Wei \& Lo, 2006). The mobile phone was pertinent in the early days of being home alone with the baby:

If it's to do with Saul, like actually the first weeks were with some breastfeeding things I used to text girls that I had known who had just had babies, they were really good and they would get back with what they thought and then you just pretty much used what works for you ... in the end I didn't need anything of that advice because he was fine. (Henrietta)

One of the points the women made about utilising the mobile was the ease with which they were able to text acquaintances that they may not have contacted personally. There was a slight irony that mobiles brought a sense of staying closely connected while at the same time 
(and in different circumstances) having enough distance to feel confident to contact people who were not so close. Socialising without the baby for the first time was daunting for the women, so texting was a functional way of staying in touch which was essential. Texting was instantaneous, discreet and allowed the women to feel secure that they were immediately accessible:

The first time I went out I found it hard to be away from her, so I would always be calling and texting to see if she was okay. (Elegi)

For all the support that enveloped the women, they still described feelings of being overwhelmed, unprepared, uncertain, and being completely physically exhausted which led to the experience of temporary gloom.

\section{The emotions}

The women were asked to describe emotions they expected would emerge during the first few months of motherhood. All the women expressed an expectation they would have mixed feelings of happiness and sadness. The uncertainty around how they would be feeling was understandable, and the stories and observations of other people's experiences contributed to their expectations. Elegi was clear about how she would feel.

I mean I think I'm going to be ... you know some days I'm going to be down, you know and then sometimes I'm going to be real happy but I think that's normal but I don't think that's got anything to do with having a baby, can't blame myself for being angry 'cause I've got a baby, I think it's all normal. (Elegi)

Elegi did not want to blame her baby for any emotional distress. Instead she categorised feelings of sadness as a normal process of motherhood; thus she normalised negative feelings just as she normalised positive feelings. Geraldine based what she expected to feel on her observation of her own mother's emotional experiences when she had her younger sister and felt that she too would be "up and down constantly" and "tired". The women found it difficult to describe only one or two emotions during the early stages of motherhood as they were as Geraldine said "expecting a ball of emotions".

All of the women were familiar with the terms "baby blues" and postnatal depression. While the terms conjured up different things for each of the women their emotional experiences were not at the severe end of the spectrum. Besides their youth which is a known risk, each of 
the women experienced life issues that, according to literature, were potential "risk factors" for inability to cope and prevalence to postnatal depression. These factors were present sometimes immediately after the birth of their baby or soon after: the death of a close relative, baby born with a physical disability, end of a relationship (i.e. single motherhood), moving home, preparing to emigrate, pregnant again two months post-birth and traumatic birth experiences. The women recounted their personal crises and described their emotional positions varying from "cry(ing) for no reason" to "being in a dark place". The women described these times as quite confusing and even though they were able to articulate their emotions vividly, they were also very proactive in finding coping strategies to help themselves move away from emotional states which were described as being an extension of the "baby blues".

Considering the women experienced at least one, and sometimes two, of the risk factors mentioned above in addition to the myriad of common adjustments in the transition to motherhood, none of the women sought to be clinically assessed or managed for depression. Elegi labelled her crying as "baby blues" but also linked it to being physically unwell:

I just used to cry for no reason like if I was finding it hard to settle her and stuff, like that I would just cry and think to myself what am I doing wrong... plus I was getting the hot and colds, I don't know why and had to go to hospital as well cause I had mastitis... so I was just getting really, not coping well... after a while I got used to it and I got over it. (Elegi)

Similarly Geraldine described her situation against a backdrop of other emotions. The well meaning advice did not decrease the anxiety she was feeling:

I remember my mum saying in the beginning, kind of like, you're out of yourself and you're just a bit of a machine, she goes give it another maybe another couple of months and then you'll come back, you'll be okay ... It was constant like anxious, I was constantly depressed and exhausted and angry, I think my life had kind of just changed automatically, I was in a dark place... I wasn't really bonding with baby as well and I just wasn't sleeping or eating... (Geraldine)

Geraldine did not get support from her immediate family as they felt what she was experiencing would pass with time and it was perceived as a weakness. Neither did they offer any alternative other than advising her to deal with it directly. As with her experience with breastfeeding, Geraldine again sought support outside of her family. Interestingly, Geraldine's 
friends advised her to seek medical attention for her depression; while this had been discouraged by her own family, it was supported by her Tokelauan mother-in-law.

An appointment was made with Geraldine's GP to discuss her emotional state but she decided not to attend as she did not want to be prescribed antidepressants. In a practical move, her mother-in-law arranged to have her baby and partner stay at her home for the weekend which allowed Geraldine time to herself. Geraldine used the time to meet up with friends, sleep, spend "all day in the sun", and read. This proved to be very successful in decreasing her extreme sadness, which lasted only two weeks thereafter, and the arrangement became an ongoing monthly fixture. Traditionally, in Pacific cultures it is expected that women will rest for at least 4 weeks, sometimes up to 6 months to enable to the women to get their bodies back in sync after birth and realign with their minds (Becker, 1995). This holistic approach expects the women to completely recuperate which means no expectation for the women to carry out work that will compromise their convalescing. The women themselves identified time alone as a priority.

\section{Time-out}

The women talked about the importance of time-out away from the baby, not only for themselves but with their partners. All the women were fortunate to have people around them who were more than willing to take care of the baby however, not everyone needed time-out. This said, all the women concluded this was an important part of the women's coping strategies:

Yep, definitely, oh my gosh it was so awesome just having a shower cause that was time on your own and you think oh my gosh is it just me ... I noticed that in the first few weeks, it's quite funny eh you take those things for granted and then when you go and you're on your own and you go whoa this is good, I definitely recommend ... even 10 minutes out of your day for yourself is so important just to refresh yourself and back to it ... yeah definitely I have tried to have that time on my own. (Henrietta)

Despite the emotional instability articulated by the women, their ability to navigate to a place where they were comfortable helped them overcome temporary states of mild "depression" (for example Geraldine felt getting her "own voice" and having less expectations of herself increased her confidence, while Elegi drew from her child's dependence on her as she now had responsibility for someone else). The women were able to move away from a place that 
compromised their health and wellbeing with the support of their family, partners and friends. The support networks were central and allowed the women to view their current situations and their future optimistically.

\section{"Everything was positive"}

Clearly, from the women's own stories not everything was positive; however the standpoint the women took to view their situations was positive. Geraldine's feelings were echoed by the other women.

I think everything was positive, even the bad things that I went through that kind of made me mature and grow up and I think that made me a better mother. I think if everything had been a breeze I would have just been an older version of my previous self which may not have been a great mum... everything kind of strengthened me and it's all been really positive. (Geraldine)

While motherhood brought on a new status for the women, it also brought an inner strength and the ability to cope through difficult situations. Geraldine's reflection highlighted that even the "bad" things contributed to her being able to mediate successfully the demanding situations that pregnancy, childbirth and motherhood bring.

\section{SUMMARY}

Returning home from hospital as a mother brought unexpected changes to the women, particularly during the early days when establishing different routines (for example feeding, sleeping) while recuperating physically was challenging. The women showed an immense amount of resilience to situations that were considered negative for example: Geraldine and the inability to attain breastfeeding; Elegi and the death of her grandmother; Henrietta and the challenges with a baby with a disability. They illustrated the ability to mediate between Samoan and New Zealand systems with ease. The world the women knew pre-birth had changed to something that now held different meanings. The women exhibited personal strength and resourcefulness and continued to explore the possibilities of support outside of their own networks.

The findings indicate the importance of support for breastfeeding and the need for more support for those who bottle-feed their babies. Glenda Wall believes the assumption that "breastfeeding is natural for women and that women, as a universal category, have the 
inherent capacity to breastfeed" (Wall, 2001, p. 597) can be detrimental to the long term mental health of the mother if she perceives herself to have failed. While breastfeeding may have been successful for three of the women in this study, it certainly was not the case for the fourth. The reasons for not breastfeeding can be the result of necessity rather than choice, yet there continue to be negative connotations attached to bottle-feeding fuelling the ever hotly debated issue (Daily Mail UK, 2010). The assumption that breastfeeding is an easy process and a rewarding experience for any motivated mother (Manhire, et al., 2007) may be the case for most women, but there are also many who encounter major difficulties that can often lead to negative emotions during the postnatal period.

Time-out was not only beneficial for the women on their own, but also with their partner, without the baby. Strong family support systems offered tangible options for the women to pursue this. Time-out was a point all the women made in order to maintain a balanced emotional wellbeing. Practical support from their families allowed the women to have "time on their own" and created a buffer against stress. The personal space offered the women a time to refocus and regain emotional and energy levels that were depleted. The time-out was not only beneficial for the women but also for their relationships.

The women did not mention midwives during the period of early motherhood which is not surprising, given that midwife care of the women ceased four-six weeks post-birth. However, the maintenance of relationships between the women and partners, family members and friends was pivotal, particularly in the provision of support and comfort.

Decisions made by all the women, empowered their mothers in a way that showed them they were positive contributors to their baby's health and wellbeing but it also showed that the women accepted and shared with the entire family a level of shared parenting. This suggests their ability to gently navigate through thorny social situations without overwhelmingly controlling the situations.

Support from other sources, particularly outside the family, was embraced by all women, who believed in accessing support where and when it was necessary. Technology including the internet and cellphone, provided support options the women could utilise and played an integral role in keeping the women connected, particularly during the very early days when they were at home on their own. 
Considering the vast amount of support surrounding the women, it did not buffer the baby blues or self-diagnosed onset of postnatal depression, and although the women were upfront about these emotions, they moved away from that place of "sadness" fairly quickly and smoothly. The women were able to sophisticatedly select the available options they needed to help them to overcome their sadness. This was achieved by evaluating their situation, assessing the options, accepting or rejecting options before taking the necessary action; this in turn obtained or maintained their resilience. Furthermore, although family, friends, partners could not protect them from the onset of baby blues, they continued to play a role in the recovery.

\section{Final words from the women}

The women were asked if there was anything from their experience they would want to share with other New Zealand-born women that they think would be beneficial for them to know about motherhood. Maintaining self-confidence to make decisions; accessing help or support immediately; and self-care were deemed the most important:

I'm just thinking of Samoan families, tell them straight up who you don't want to be in the room... I didn't really have that problem, but you know I've heard stories... or have someone there who can do it for you if you don't want to cause too much tension in the family, having someone there, maybe have your midwife to tell them to get out... in Samoan families mothers know best I think, they do have good advice even if you're not close to your mum. (Henrietta)

Just don't stress out if you need help just ask someone, don't just hold on to it and just stress out 'cause some ladies or I hear that some ladies they can't take it ... they just leave their babies crying and they'll cry themselves as well, don't do that go to someone and talk to them or even ask Plunket to help you out or even join the ladies and mothers group, that should be able to help out. (Alma)

That motherhood is hard... don't believe what people tell you about their births cause it's always going to change, it will be different for yourself... well, I think maybe when she was much younger I didn't really get time out and stuff which I do now which has helped a lot, 'cause I wanted to do it myself, try and handle her myself but I got through that when I kept getting told that I need to have time out otherwise I'm going to just stress myself. (Elegi)

I'd tell them to assess where they're at and if they feel like it's really hard for them to cope with or if they kind of feel like something might happen to baby or themselves then I would tell them to go to the doctor straight away and look for that support and if they're not getting it from home then to look elsewhere because they need to take care of their child and themselves. (Geraldine) 


\section{CHAPTER SEVEN: CONCLUSION}

This research explored in depth the experiences of four New Zealand-born Samoan first time mothers. A bricoleur approach was used which included feminist qualitative methods to capture the women's stories in their own words. This thesis provided an opportunity to share in the lives of four women during an event which was both life changing and life challenging. The women were part of two - some would consider colliding - worlds and in most cases, overcame the many difficulties they encountered. In summarising the answers to my research questions I will briefly describe the key factors identified by the women themselves that contributed to their health and wellbeing. As this is a strengths-based study I will highlight areas where the women demonstrated their ability to view their outcomes as positive - and their ability to mediate successfully between the offerings of Samoan culture and New Zealand health systems.

\section{Sophisticated Mediators}

Findings of this study form the basis for constructing a Sophisticated Mediator theory that relates specifically to the four New Zealand-born Samoan women of this study. The emergence of data from the narratives of the women redefined the concept of sophisticated mediators as it was first proposed by African-American feminist researcher, Patricia Hill Collins. Hill Collins coined the phrase "sophisticated mediators" to describe effective Black mothers and the choices and strategies they employed in raising their children between "an oppressive culture and a nurturing Black value-structure" (Hill Collins, 1986, p. S22). While respectfully acknowledging Hill Collins's definition of sophisticated mediators, in redefining the concept I have deromanticised the term as I do not claim that Samoan values are nurturing whilst the New Zealand culture is oppressive; rather, that the two cultures are treated as neutral and it is demonstrated that the women go back and forth between them.

"Sophisticated Mediators" thus exemplifies the way the women were able to negotiate successfully between the two cultures. Mediating successfully meant the women remained resilient to many adverse factors which led ultimately to positive outcomes during pregnancy, childbirth and the transition to motherhood. In their construction and understanding of what motherhood meant they articulated a world where, although some things did not make sense, they were able to adapt to situations in a way that preserved their resilience. The 
Sophisticated Mediators concept worked at different levels: social and individual. Illustrated in the many examples from the findings, the women were adept at selecting from the offerings of both worlds, rather than trying to fit into one or the other worlds. The women mediated relationships; mediated between relationships; mediated negative situations and views in order to see the positives.

"Sophisticated Mediators" is a concept that located the women in a position of strength rather than a position of weakness/deficit. The women of this study approached pregnancy, childbirth and early motherhood from a place where they were able to identify, articulate, facilitate and find solutions towards positive outcome for their own health and wellbeing. While the women were challenged many times during the maternal period, the challenges and possible barriers were negotiated in a way that illustrated the high level resolution employed by them to reconcile the health and wellbeing resources available and to satisfy their needs, both physical and emotional. Sophisticated mediation meant being open to accept both Samoan and New Zealand cultures and value systems without the need to give one up for the other. As a researcher who entered into the women's lives at a specific point in their life cycle, I can only postulate that this ability and skill as "Sophisticated Mediators" was cultivated from birth and continues to develop and progress.

The overall research question was: What are the experiences of a group of first-time New Zealand-born Samoan mothers before and after birth?

The research aim was to identify factors that may affect or contribute to the health and wellbeing of New Zealand-born Samoan women during pregnancy and post-birth.

My first aim in this research was to identify a range of support systems utilised by Samoan women during pregnancy and up to 12 months post-birth. Literature focussing on the maternal health of women highlights the significance of social support on the overall wellbeing of women, particularly during the early days of motherhood. For this thesis it was important to identify not only the support systems available to New Zealand-born women but also which support systems the women in this study were utilising.

The support systems were divided into two categories, informal and formal, and the establishment of these support systems was an immediate reaction to the confirmation of their pregnancies even though most of the support networks existed before their pregnancies i.e. 
family and friends while others were new i.e. midwives. The women strongly stated their responsibility for their pregnancies, which for three were unplanned, and they were proactive about informing themselves about impending childbirth and motherhood. As several other researchers found, aspects of the family construct served as both protective and risk factors. For example, Geraldine's father provided her with "nurturing" at the actual birth; however, her family's dismissal of her self-diagnosed postnatal depression left Geraldine feeling isolated. The women utilised their informal support systems which included family immediate and extended, friends and acquaintances and these were considered an important source of support for all the women of this study throughout the maternity process. In particular, the women developed solid relationships with their mothers and/or another significant other female person and this not only provided practical support but also emotional support in the form of a confidante that could be trusted.

The frequent referral to the importance of family members was not surprising considering "family" was mentioned throughout both interview phases as being an integral part of pregnancy, childbirth and motherhood. Formal support networks included midwives, community health centres and, surprisingly, technology i.e. the internet and mobiles/cellphones for texting and telephone based support. Technology played a significant role in supporting the women throughout the pregnancy, childbirth and early motherhood period in that it kept them connected with friends, family or support groups at a time when it was inconvenient or physically impossible to meet up in person. It should be noted that mothers' groups, i.e. morning tea groups, antenatal class get togethers or Plunket PIN groups, were not a source of support for any of the women in this research. When asked directly about these groups the women were not interested in socialising further with couples from their antenatal groups or being part of a new mothers' group; however, no rationale for this decision was provided.

Another aim was to compare the expectations they held about childbirth and motherhood with their actual experiences. To enable this it was imperative for all the women to be first time mothers so that their expectation of their experiences would not be influenced by previous experiences. The women's expectations of childbirth and early motherhood did not match their experiences; this was particularly evident for childbirth where stark contrasts were revealed e.g. "I expect birth will be easy" but the reality was that birth was difficult. This is a similar finding to other studies (for example see Gibbins \& Thomson, 2001; Mann, 
et al., 1999; Rice \& Naksook, 1999) where a mismatch between expectations and experiences occurred. Some studies found this to be a risk factor as women became disillusioned with the maternity experience which then led to emotional distress. However, this was not the case for the women of this study; when the experience did not match the expectation it was seen as an obstacle to overcome, something that strengthened the woman's character and therefore it was perceived as a positive event.

Another aim was to identify coping strategies Samoan women employ for the first 12 months post birth. Firstly, the women were very skilful at identifying a need and weighing up the options available to meet that need, before deciding on the best approach. For example: during her birth, Geraldine needed emotional nurturing which she felt only her father could provide, therefore, she summoned him to attend the birth; Alma, frustrated and disappointed at the unsatisfactory treatment she received from her midwife, chose to resolve the issue in a manner that may have been construed as culturally inappropriate.

The coping strategies included the ability to turn what potentially could be a negative situation into a positive outcome. When the women held different beliefs and value systems from their families they accessed support outside of the family networks to cope with the situation at hand, for example seeking support from outside agencies to assist with breastfeeding issues and postnatal depression.

A further aim was to gain an understanding of the impact of motherhood on the emotional wellbeing of Samoan women, which was significant, and although the women were expecting changes in their lives they were not fully prepared for the major effect motherhood had on their emotional wellbeing. "Baby blues" and self-identified early postnatal depression was part of the experience described by the women.

The key factors identified by the women that affected or contributed to their health and wellbeing included:

Solid and reliable support networks which ensured the women felt confident they were well supported, in particular the personal relationships between mother-daughter and her significant other female role models; and the relationships with the woman 
and her partner. The women were acutely aware of the importance of maintaining these connections as they contributed to attaining positive outcomes.

Maintenance of perceived autonomy over their personal situations, particularly during childbirth, was one of the key factors that influenced the health and wellbeing of the women. Planning and organising (for example birth plans, maternity leave, gathering information) was a way the women felt they were taking full responsibility for their earlier than expected path to motherhood.

$>$ The women accepted that their experiences did not necessarily match up with their expectations of childbirth, or equally early motherhood.

$>$ The ability to ask for help. Whilst this was initially difficult for one of the women, it became relatively easy as time passed. The women were not averse to asking for help and saw it as a coping strategy to testing situations.

Families, which are very much considered part of the upbringing of the children rather than just support for the mothers. While their influence is within the realms of what is considered appropriate by the women, the women felt the families were respectful of these boundaries. The families articulating they "want" to be part of the lives of the babies in a meaningful way also contributed to the confidence of the women in asking for help.

\section{Recommendations}

The following recommendations aimed at maternal health care providers, family, friends and first-time mothers, are based on my research findings and interviews with Alma, Elegi, Geraldine and Henrietta. The current ad hoc manner of women securing the services of a midwife was not conducive to the women's wellbeing or confidence and this system should be revised and refined in order for all women to have better options, i.e. a preference for one midwife or a team of midwives should be catered for. Another strong recommendation from the women was that visitors should wait at least a week before visiting mothers, particularly first-time mothers. Time is needed to adjust to the new baby, the battered body and the constant exhaustion and tiredness the women were feeling during the early days at home. In addition, the desire to establish a feeding regime was crucial and visitors were, unintentionally, intruding on and hindering this process. Bottlefeeding should not be frowned upon and more support and information about the option of bottle feeding should be more readily accessible. Technology played a central part in social connectedness and service 
providers should utilise the internet and texting more in order to provide information or keep in touch with new mothers.

\section{Risk factors}

The adverse situations the women faced during the time-span of the research were considered "risk factors" for postnatal depression and each of the participants experienced at least one: death of a close family member; break up of a relationship; birth of a child with a severe disability; a second pregnancy when the first child was 2 months old; breastfeeding issues; relocating i.e. moving house or country; cohabitating. However, not all the women experienced postnatal depression.

I attempted to identify and contextualise the pinnacle of the women's resilience to risk factors and my research led me to conclude that the resilience of the women of this study is due to the fact that they appeared to challenge or question what New Zealand or Western society consider "normal". What is "not normal" during pregnancy, childbirth and motherhood is considered a "risk factor". The women were aware of the impact the risk factors had on their lives but were able to normalise these risks and therefore decrease the negative impact. For example, one participant experienced a relationship break-up, yet she normalised this experience and thereby lessened its negative impact. The women accepted that while "risk factors" were not ideal they were obstacles to be overcome and therefore they proceeded to manage as best they could and move on. Inability to breastfeed? Sad, but bottlefeeding is okay. Death in the family? Very sad but a normal part of the life cycle. The women completely embraced motherhood, warts and all.

The method of conducting two interviews per woman at different points of time (for example: Interview 1 at the last trimester of pregnancy; and Interview 2 at up to 12 months post-birth) proved to be beneficial for this study as it provided the opportunity to observe changes in the women's attitudes as they progressed through their pregnancies and post birth, and allowed fascinating insights into the women's lives. A particularly interesting observation was the psychological and emotional changes to the women as they transited to motherhood; notably that, by the second interview, the women were more self-confident, more forthcoming and more willing to share their opinions, views and their own stories. They appeared more aware of their personal situation in relation to their families, communities and society; this impacted, for example, on how they viewed and planned for their futures. Alma, as a single 
parent, decided to return to full-time study in order to provide a secure, financial future for herself and her daughter. Both interviews also provided an insight into the establishment and development of relationships during pregnancy through to early motherhood.

\section{Research implications:}

This research has opened the way for various areas of further investigation into the experiences of pregnancy, childbirth and motherhood and their contribution or influence on the emotional wellbeing of young women. This is particularly relevant not only within a New Zealand context but also internationally. The outcomes of this research revealed an interesting concept of Sophisticated Mediators and begged the question of whether this was an artefact of this particular group of women and whether this concept, indeed, contributed to the lower rates of postnatal depression among New Zealand-born Samoan women. While this study was limited to women under 30 years of age and all four were New Zealand-born Samoans living in the same urban area, future research exploring the experiences of women over 30 years living in cities as well as semi-rural and rural areas might highlight significant differences in outcomes. This research demonstrated the critical significance of the motherdaughter relationship and the impact this had on the health and wellbeing of the women. It would be interesting to explore further the pregnancy and childbirth experiences of the women's mothers (or significant other female) and the relevance of this for their daughters' experiences.

\section{Limitations:}

All the women in this study were first time mothers which may have produced a more positive attitude towards motherhood--not to mention the positive status that motherhood afforded them. In addition, as first time mothers none of the women were responsible for any other children of their own. However, it is acknowledged that some of the women had stepchildren and younger siblings included in their own immediate families, i.e. living in the same residence. Therefore, future research is required to investigate whether women who have more than one child have the same abilities to initiate coping strategies and successfully mediate as was illustrated by the women of this study.

All the women were relatively young; three were 20 years old and one was 29 years old. It would be interesting to explore the experiences of women who are $30+$ as their support systems and need for support may differ - i.e. to explore whether the mother-daughter 
relationship would be as important to older mothers as it was to the women in this study. The sample size for this research was relatively small and, while I argue that the sample size produced rich, descriptive stories of the participants' experiences, future research with a larger and more wide-ranging sample will be beneficial. This research explored the expectations and experiences of pregnancy, childbirth and early motherhood of four New Zealand-born women living in Wellington. Future research could test these findings using a larger sample base of Samoan women of different ages, countries of birth and from different geographic locations.

\section{Final words}

The women in this study were Sophisticated Mediators - successfully mediating between their inherited Samoan value and belief system and also the New Zealand value and belief system which they also had access to as New Zealand-born Samoans. The opportunity and freedom to evaluate and select the most appropriate offerings from both these cultures helped to develop strong, independent and confident women. Undoubtedly, the women were faced with risk factors that temporarily clouded their experience. However, the words or terms stated in Chapter Two which are often used to describe the New Zealand-born Samoan experience such as "struggle", "dilemma", an "identity crisis", "bridging the cultures", "mediating discontinuous worlds", "fitting in" were not applicable to these women.

The outcomes of this project have instigated a continuation of further contributions to the knowledge around the experiences of first time Samoan mothers before and after birth and the coping strategies adopted by these mothers post birth. The research identified key factors relating to positive health and wellbeing outcomes for Samoan women and provided recommendations for policy and practice for government agencies, including the delivery of culturally appropriate maternity services for women.

In conclusion, the women were confident in their identity as New Zealand-born Samoan and their self defined cultural positioning was important. The women felt others, particularly the older generation, needed to accept, acknowledge and value the standpoint of the New Zealand-born generation. The lack of language skills and knowledge of Samoan culture did not equate to being any less "Samoan", and they took pride in identifying themselves as New Zealand-born Samoan. 
Furthermore, my quest to understand how New Zealand-born Samoan women construct pregnancy, childbirth and motherhood transpired into abundant and vivid descriptions of the women's experiences from their perspective. Their ability and motivation to effectively manage and negotiate their lives for the health and wellbeing of themselves and their babies and families was outstanding, particularly as it occurred during a time of constant change. I submit this thesis with great hope that it contributes to furthering the knowledge around the topic of postnatal depression but also, importantly, that I have captured the women's stories in a respectful manner. 


\section{REFERENCES}

Abbott, M. W., \& Williams, M. M. (2006). Postnatal depressive symptoms among Pacific mothers in Auckland: prevalence and risk factors. Royal Australian and New Zealand College of Psychiatrists.

Abel, S., Park, J., Tipene-Leach, D., Finau, S., \& Lennan, M. (2001). Infant care practices in New Zealand: a cross-cultural qualitative study. Social Science and Medicine, 53, 1135-1148.

Agnew, F., Pulotu-Endermann, F. K., Robinson, G., Suaalii-Sauni, T., Warren, H., Wheeler, A., et al. (2004). Pacific Models of Mental Health Service Delivery in New Zealand ("PMMHSD") Project. Auckland: Ministry of Health, Health Research Council of New Zealand, and Mental Health Commission.

Airini, Anae, M., Mila-Schaaf, K., Coxon, E., Mara, D., \& Sanga, K. (2010). Teu Le Va Relationships across research and policy in Pasifika education: A collective approach to knowledge generation and policy development for action towards Pasifika education success. Wellington: Ministry of Education.

Anae Fuamatu N., Lima, I., Mariner, K., Park, J., \& Suaalii-Sauni, T. (2000). Tiute ma Matafaioi a nisi Tane Samoa i le Faiga o Aiga. The roles and responsibilities of some Samoan men in reproduction. Auckland: Pacific Health Research Centre. The University of Auckland.

Anae, M. (2002). Papalagi Redefined: Toward a New Zealand-born Samoan Identity. In P. R. Spickard, J. L. Rondilla \& D. H. Wright (Eds.), Pacific diaspora: island peoples in the United States and across the Pacific. Hawai'i: University of Hawai'i Press.

Asiasiga, L. (1994). Abortion and Pacific Islands Women: A pilot study for the New Zealand Family Planning Association. Wellington: New Zealand Family Planning Association.

Asiasiga, L. (2007). Influential factors shaping social science research about Pacific peoples. Unpublished PhD Thesis. Victoria University of Wellington.

Baca Zinn, M. (1979). Field research in minority communities: ethical, methodological and political observations by an insider. Social Problems, 27(2), 209-219.

Baker, S. R., Choi, P. Y. L., Henshaw, C. A., \& Tree, J. (2005). 'I felt as though I'd been in Jail': Women's experiences of maternity care during labour, delivery and immediate postpartum. Feminism \& Psychology, 15(3).

Barclay, L., Aiavao, F., Fenwick, J., \& Papua, K. T. (2005). Midwives' Tales: Stories of traditional and professional birth in Samoa. Nashville: Vanderbilt University Press.

Becker, A. E. (1995). Body, Self, and Society: The view from Fiji. Pennsylvania: University of Pennsylvania Press.

Beoku-Betts, J. (1994). When black is not enough: doing field research among Gullah women. National Women's Studies Association Journal, 6(3), 413-433.

Berking, T., Fatialofa, C. S., Lupe, K., \& Skipps-Patterson, S. (2007). Being 'Afakasi. In P. Culbertson, M. N. Agee \& C. O. Makasiale (Eds.), Penina Uliuli: Contemporary challenges in Mental Health for Pacific Peoples. Honolulu: University of Hawai'i Press.

Bird, L., \& Drewery, W. . (2004). Human development in Aotearoa. Sydney: McGraw-Hill.

Burns, E., Schmied, V., Sheehan, A., \& Fenwick, J. (2009). A meta-ethnographic synthesis of women's experience of breastfeeding. Maternal and Child Nutrition.

Butler, S., Williams, M., Tukuitonga, C., \& Paterson, J. (2004). Factors associated with not breastfeeding exclusively among mothers of a cohort of Pacific infants in New Zealand. The New Zealand Medical Journal, 117(1195). 
Byrne, M. M. (2001). Understanding life experiences through a phenomenological approach to research. Retrieved September, 2008, from http://findarticles.com/p/articles/mi_m0FSL/is_/ai_73308177

Campbell, R., \& Wasco, S. M. (2000). Feminst approaches to social science: epistemological and methodological tenets. American Journal of Community Psychology, 28(6), 773791.

Charmaz, K. (2000). Grounded theory: Objectivist and Constructivist methods. In N. K. Denzin \& Y. S. Lincoln (Eds.), Handbook of Qualitative Research (2nd ed., pp. 509535). Thousand Oaks: Sage.

Churchward, M. (2010). The health and wellbeing of New Zealand-born Samoan women during pregnancy, childbirth and early motherhood: UNPUBLISHED DEMOGRAPHICS OF THE PARTICIPANTS. Victoria University of Wellington.

Collins, B. (2005). Missing voices: teenage pregnancy and social policy. Unpublished $\mathrm{PhD}$ Thesis. Victoria University of Wellington.

Cox, J. L., Holden, J. M., \& Sagovsky, R. (1987). Detection of postnatal depression: Development of the 10-item Edinburgh postnatal depression scale. British Journal of Psychiatry(150), 782-786.

Crotty, M. (1998). The foundations of social science research: meaning and perspective in the research process. Crows Nest: Allen and Unwin.

Crouch, M., \& Manderson, L. (1993). New motherhood: Cultural and personal transitions in the 1980s. Victoria, Australia: Gordon and Breach Science.

Crouch, M., \& McKenzie, H. (2006). The logic of small samples in interview-based qualitative research. Social Science Information, 45(4), 483-499.

Daily Mail UK. (2010). Mothers demand apology after parenting magazine writer calls breastfeeding 'creepy'. Daily Mail UK.

Davidson, C., \& Tolich, M. (2007). Competing traditions. In C. Davidson \& M. Tolich (Eds.), Social Science Research in New Zealand: Many paths to understanding (2nd ed.). 2007: Pearson Education New Zealand.

De Andrade, L. L. (2000). Negotiating from the inside: Constructing racial and ethnic identity in qualitative research. Journal of Contemporary Ethnography, 29(3), 268-290.

Denzin, N. K., \& Lincoln, Y. S. (2000). Introduction: The discipline and practice of qualitative research. In N. K. Denzin \& Y. S. Lincoln (Eds.), Handbook of Qualitative Research (2nd ed.). Thousand Oaks: Sage.

Denzin, N. K., \& Lincoln, Y. S. (Eds.). (2008). Handbook of Critical and Indigenous Methdologies. CA: Thousand Oaks.

Donnelly, P. (1992). Samoan womens' voices: Alternative ways of doing birth. Unpublished $\mathrm{PhD}$ Thesis. University of Otago, Dunedin, New Zealand.

Dwyer, S. (2009). Childbirth education: antenatal education and transitions of maternity care in New Zealand. Wellington: Families Commission.

Ekeroma, A., Craig, E. D., Stewart, A. W., Mantell, C. D., \& Mitchell, E. A. (2004). Ethnicity and birth outcome: New Zealand trends 1980-2001: Part 3. Pregnancy outcomes for Pacific women. Australian and New Zealand Journal of Obstetrics and Gynaecology(44), 541-544.

Ekeroma, A., \& Harillal, M. (2003). Women's choice in the gender and ethnicity of her obstetrician and gynaecologist. Australian and New Zealand Journal of Obstetrics and Gynaecology(43), 354-359.

Ermine, W., Sinclair, R., Jeffery, B., \& Indigenous Peoples' Health Research Centre. (2004). The Ethics of Research involving Indigenous Peoples. Canada: Indigenous Peoples' Health Research Centre. 
Evans, R., \& Becker, S. (2009). Parents with HIV and Aids: Global issues and policy responses. Bristol, United Kingdom: The Policy Press.

Fa'asalele Tanuvasa, A. (1999). The place of contraception and abortion in the lives of Samoan women. Unpublished $\mathrm{PhD}$ Thesis, Volume 1. Victoria University of Wellington.

Fairbairn-Dunlop, P. (1998). Tamaitai Samoa: Their stories. Suva: Institute of Pacific Studies, The University of the South Pacific.

Fairbairn-Dunlop, P., \& Makisi, G. (2003). Making our place: Growing up PI in New Zealand. Palmerston North: The Dunmore Printing Company Limited.

Families, K. (2010). BirthPlan. Retrieved March, 2010, from http://www.kiwifamilies.co.nz/Topics/Birth/Birth-Plan.html

Finfgeld-Connett, D. (2005). Telephone social support or nursing presence? Analysis of a nursing intervention. Qualitative Health Research, 15(1), 19-29.

Forbat, L., \& Henderson, J. (2005). Theoretical and practical reflections on sharing transcriptions with participants. Qualitative Health Research, 15(8), 1114-1128.

Forster, D., McLachlan, H., Rayner, J., Yelland, J., Gold, L., \& Rayner, S. (2008). The early postnatal period: Exploring women's views, expectations and experiences of care using focus groups in Victoria, Australia. BMC Pregnancy and Childbirth, 8(1), 27.

Gegeo, D. W. (1998). Indigenous knowledge and empowerment: Rural development examined from within. The Contemporary Pacific, 10(2), 289-315.

Gibbins, J., \& Thomson, A. M. (2001). Women's expectations and experiences of childbirth. Midwifery(17), 302-313.

Goodman, P., Mackey, M. C., \& Tavakoli, A. S. (2004). Factors related to childbirth satisfaction. Journal of Advanced Nursing, 46(2), 212-219.

Griffen, V. (1983). Care for ourselves: A health handbook for Pacific women. Suva: University of the South Pacific.

Guba, E. G., \& Lincoln, Y. S. (2000). Paradigmatic controversies, contradictions, and emerging confluences. In N. K. Denzin \& Y. S. Lincoln (Eds.), Handbook of qualitative research (2nd ed., pp. 163-188). Thousand Oaks: Sage.

Hale, J. (1980). The black women and child rearing. In L. Rodgers-Rose (Ed.), The Black Women (pp. 79-88). Beverly Hills, CA: Sage.

Hattori, A. P. (2006). 'The Cry of the Little People of Guam': American Colonialism, Medical Philanthropy, and the Susana Hospital for Chamorro Women, 1898-1941. Australian and new Zealand Society of the History of Medicine, 8(1), 4-26.

Health Research Council of New Zealand. (2005). Guidelines on Pacific Health Research. Auckland.

Hereniko, V. (2000). Indigenous knowledge and academic imperialism. In R. Borofsky (Ed.), Remembrance of Pacific Pasts: An invitation to remake history (pp. 79-91). Honolulu: University of Hawai'i Press.

Hill Collins, P. (1986). Learning from the outsider within: The sociological significance of Black feminist thought. Social Problems, 33(6), 175-191.

Hill Collins, P. (1989). The social construction of black feminist thought. Journal of Women in Culture and Society, 14(4), 745-773.

Hill Collins, P. (1990). Black feminist thought in the matrix of domination Black feminist thought: knowledge, consciousness, and the politics of empowerment (pp. 221-238). Boston: Unwin Hyman.

Hill Collins, P. (1998). It's all In the family: intersections of gender, race, and nation. Hypatia, 13(3), 62-82.

Hill Collins, P. (1999). Reflections on the outsider within. Journal of Career Development, 26(1), 85-88. 
Hodnett, E. (2002). Pain and women's satisfaction with the experience of childbirth: A systematic review. Americal Journal of Obstetrics and Gynaecology, 186(5), S160S172.

Hunkin-Tuiletufuga, G. (2001). Pasifika languages and Pasifika identities: Contemporary and Future challenges. In C. Macpherson, P. Spoonley \& M. Anae (Eds.), Tagata OT Moana Nui: The Evolving Identities of Pacific Peoples in Aotearoa/New Zealand. Palmerston North: Dunmore Press.

Kerslake-Hendricks, A. (1998). Postnatal support used by first-time mothers. Unpublished Master of Arts (Applied) Social Science Research. Victoria University of Wellington.

Kingi-Uluave, D., \& Olo-Whaanga, E. (2010). Talking therapies for Pasifika Peoples: best and promising practice guide for mental health and addiction services. Auckland.

Kinloch, P. (1985). Midwives and midwifery in Western Samoa. In C. D. F. Parsons (Ed.), Healing practices in the South Pacific. Hawaii: University of Hawaii Press.

Kitzinger, C. (2005). Heteronormativity in action: Reproducing the heterosexual nuclear family in after-hours medical calls. Social Problems, 52(4), 477-498.

Knight, R., \& Thirkettle, J. A. (1987). The relationship between expectations of pregnancy and birth, and transient depression in the immediate post-partum period. Journal of Psychomatic Research, 31(3).

Kvale, S. (1996). Interviews: An introduction to qualitative research interviewing: Sage, Thousand Oaks.

Kvale, S., \& Brinkmann, S. (2009). InterViews: Learning the craft of qualitative research interviewing (2nd ed.): Sage.

Ladson-Billings, G. (2000). Racialized discourses and ethnic epistemologies. In N. K. Denzin \& Y. S. Lincoln (Eds.), The handbook of qualitative research (2nd ed.). California: Sage Publications.

Lally, J. E., Murtagh, M. J., Macphail, S., \& Thomson, R. (2008). More in hope than expectation: a systematic review of women's expectations and experience of pain relief in labour. BMC Medicine, 6(7).

Lavender, T., Downe, S., Finnlayson, K., \& Walsh, D. (2007). Access to antenatal care: A systematic review: University of Central Lancashire.

Lia-Hoagberg, B., Rode, P., Skovholt, C. J., Oberg, C. N., Berg, C., Mullett, S., et al. (1990). Barriers and motivators to prenatal care among low-income women. Social Science and Medicine, 30(4), 487-495.

Lifflander, A., Gaydos, L. M., \& Hogue, C. R. (2006). Circumstances of pregnancy: Low income women in Georgia describe the difference between planned and unplanned pregnancies. Maternal Child Health Journal, 11, 81-89.

Logsdon, M. C., Wisner, K. L., \& Pinto-Foltz, M. D. (2006). The impact of postpartum depression on mothering. Journal of Obstetric, Gynecologic, \& Neonatal Nursing, 35(5), 652-658.

Low, P., Paterson, J., Wouldes, T., Carter, S., Williams, M., \& Percival, T. (2005). Factors affecting antenatal care attendance by mothers of Pacific infants living in New Zealand. The New Zealand Medical Journal, 118(1216).

Lui, D. (2007). Spiritual Injury: A Samoan perspective on Spirituality's impact on mental health. In P. Culbertson, M. Agee \& C. 'Ofa Makasiale (Eds.), Penina Uliuli: Contemporary challenges in Mental Health for Pacific Peoples: University of Hawai'i Press.

Macpherson, C., \& Macpherson, L. (1990). Samoan medical belief and practice. Auckland: Auckland University Press. 
Manhire, K. M., Hagan, A. E., \& Floyd, S. A. (2007). A descriptive account of New Zealand mothers' responses to open-ended questions on their breast feeding experiences. Midwifery, 23, 372-381.

Mann, R. J., Abercrombie, P. D., DeJoseph, J., Norbeck, J. S., \& Smith, R. T. (1999). The personal experience of pregnancy for African-American women. Journal of Transcultural Nursing, 10(4), 297-305.

Marsh, S. T. (1999). Migrating feminisms: Maligned overstayer or model citizen? Women's Studies International Forum, 21(6), 665-680.

McMahon, C., Barnett, B., Kowalenko, N. M., \& Tennant, C. C. (2006). Maternal attachment state of mind moderates the impact of postnatal depression on infant attachment. Journal of Child Psychology and Psychiatry, 47(7), 660-669.

McQuillan, J., Greil, A. L., \& Shreffler, K. M. (2010). Pregnancy intentions among women who do not try: focussing on women who are okay either way. Maternal Child Health Journal.

Meleisea, M., \& Schoeffel, P. (1998). Samoan Families in New Zealand. In V. Adair \& R. Dixon (Eds.), The Family in Aotearoa (pp. 285-296). Auckland: Addison Wesley Longman.

Melender, H.-L. (2002). Experiences of fears associated with pregnancy and childbirth: A study of 329 pregnant women. Birth, 29(2).

Merck, Sharp, \& Dohme Corporation. (2010). Women's Health Issues: Labour. The Merck Manuals: Online Medical Library. Home Edition: For patients and caregivers Retrieved July, 2010

Mila-Schaaf, K., \& Hudson, M. (2009). The interface between cultural understandings: Negotiating new spaces for Pacific mental health. Pacific Health Dialog, 15(1).

Milgrom, J., Gemmill, A. W., Bilszta, J. L., Hayes, B., Barnett, B., Brooks, J., et al. (2008). Antenatal risk factors for postnatal depression: A large prospective study. Journal of Affective Disorders, 108, 147-157.

New Zealand College of Midwives Incorporated. (2010). Information for Midwives and Women. Retrieved April, 2010, from http://www.midwife.org.nz/

New Zealand Government. (2008). New Zealand Legislation: Acts. Parental Leave and Employment Protection Act 1987. Retrieved June, 2010

New Zealand Ministry of Health. (2008). Maternity Action Plan 2008-2012: Draft for Consultation. $\quad$ Retrieved September, 2008, from http://www.moh.govt.nz/moh.nsf/pagesmh/8445/\$File/draft-maternity-actionplan.oct08-final.doc

New Zealand Ministry of Health. (2009). National Strategic Plan of Action for Breastfeeding 2008-2012: National Breastfeeding Advisory Committee of New Zealand's advice to the Director-General of Health.

New Zealand Ministry of Health. (2010a). Breastfeeding - The benefits of breastfeeding. Retrieved May, 2010, from http://www.moh.govt.nz/moh.nsf/indexmh/breastfeedingwhy

New Zealand Ministry of Health. (2010b). Healthline. Retrieved March, 2010, from http://www.moh.govt.nz/healthline

New Zealand Ministry of Health. (2010c). Maternity. Retrieved April, 2010, from http://www.moh.govt.nz/maternity

Ngan-Woo, F. E. (1985). Fa'a Samoa: The world of Samoans. Wellington, New Zealand: The Office of Race Relations Conciliator.

Norris, P., Fa'alau, F., Churchward, M., Va'ai, C., \& Arroll, B. (2006). Language, Culture and Antibiotics: Samoans in New Zealand and Samoa, Reports: International Social Pharmacy Workshop (pp. A32-A32). Australia: Pharmaceutical Press. 
Norris, P., Fa'alau, F., Va'ai, C., Churchward, M., \& Arroll, B. (2009). Navigating between illness paradigms: Treatment seeking by Samoan people in Samoa and New Zealand. Qualitative Health Research, 19(10), 1466-1475.

Oakley, A. (1979). From here to maternity: Becoming a mother. Suffolk: Penguin Books.

Oakley, A. (1984). The captured womb: A history of the medical care of pregnant women. New York: Basil Blackwell Publisher Ltd.

Oakley, A. (1992). Social support and motherhood. Oxford: Blackwell Publishers.

Oates, M. R., Cox, J. L., Neema, S., Asten, P., Glangeaud-Freudenthal, N., Figueredo, B., et al. (2004). Postnatal depression across countries and cultures: a qualitative study. British Journal of Psychiatry, 184 (suppl. 46), 10-16.

Paterson, J., Cowley, E. T., Percival, T., \& Williams, M. (2004). Pregnancy planning by mothers of Pacific infants recently delivered at Middlemore Hospital. The New Zealand Medical Journal, 117(1188).

Patton, M. Q. (2002). Qualitative research and evaluation methods (3rd ed.). California: Sage Publications Inc.

Pere, L. (2006). Oho mauri: Cultural identity, wellbeing, and tangata whai ora/motuhake. Unpublished doctoral thesis, Massey University, Palmerston North.

Philo, C. (2003). 'To go back up the side hill": Memories, Imaginations and Reveries of Childhood. Children's Geographics, 1(1), 7-23.

Plunket. (2010). PlunketLine. Retrieved March, 2010, from http://www.plunket.org.nz/

Plunket New Zealand. (2010). Food and Nutrition - Up to 1 year: You and your child: What to feed them by 7 or 8 months. Retrieved July, 2010

Pulotu-Endemann, F. K., Suaali'i-Sauni, T., Lui, D., McNicholas, T., Milne, M., \& Gibbs, T. (2007). Seitapu Pacific Mental Health and Addiction Cultural \& Clinical Competencies Framework. Auckland: Mental Health Research and Workforce Development.

Reinharz, S. (1992). Feminist methods in social research. Oxford: Oxford University Press.

Rice, P. L., \& Naksook, C. (1999). The experience of pregnancy, labour and birth of Thai women in Australia. Midwifery, 14, 74-84.

Richards, H. M., \& Schwartz, L. J. (2002). Ethics of qualitative research: are there special issues for health services research? Family Practice, 19(2), 135-139.

Righetti-Veltema, M., Conne-Perreard, E., Bousquet, A., \& Manzano, J. (1998). Risk factors and predictive signs of postpartum depression. Journal of Affective Disorders(49), 167-180.

Robertson, E., Celasun, N., \& Stewart, D. E. (2003). Risk factors for postpartum depression. In D. E. Stewart, E. Robertson, C.-L. Dennis, S. L. Grace \& T. Wallington (Eds.), Postpartum depression: Literature review of risk factors and interventions. Canada: University Health Network Women's Health Program 2003.

Robertson, E., Grace, S., Wallington, T., \& Stewart, D. E. (2004). Antenatal risk factors for postpartum depression: a synthesis of recent literature. General Hospital Pyschiatry(26), 289-295.

Rutter, M. (1999). Resilience concepts and findings: implications for family therapy. The Association for Family Therapy, 21, 119-144.

Ryan, D., \& Ekeroma, A. (2010). Personal Communication: Validity of the Edinburgh Postnatal Depression Scale (EPDS). Wellington.

Safuneituuga, P. N. (2005). Statement on behalf of the Government of Samoa. Forty-ninth session of the Commission on the status of Women. Retrieved from www.un.org/webcast/csw2005/statements/050307samoa-e.pdf

Sarantakos, S. (2005). Social Research (3rd ed.). New York: Palgrave MacMillan. 
Smith, L. T. (1999). Decolonizing Methodologies: research and indigenous peoples. London and Dunedin: Zed Books and University of Otago Press.

Southwick, M. (2001). Pacific women's stories of becoming a nurse in New Zealand: A radical hermeneutic reconstruction of marginality. Unpublished $\mathrm{PhD}$ Thesis. Victoria University of Wellington.

Statistics New Zealand. (2004). Household access to the internet. Wellington: Statistics New Zealand.

Statistics New Zealand. (2006). Population of New Zealand, 2006 Census. Retrieved January, 2010, from http://www.stats.govt.nz/Census/2006CensusHomePage/QuickStats/AboutAPlace/Sna pShot.aspx?id=9999999\&type=region

Statistics New Zealand. (2008a). Demographic Trends: 2007. Retrieved September, 2008, from http://www.stats.govt.nz/NR/rdonlyres/6476A1DB-472E-412A-98900C09A3B2ED37/0/Chapter8 Nationaldemographicprojections.pdf

Statistics New Zealand. (2008b). Pacific Peoples: A Snapshot. Retrieved May, 2008

Statistics New Zealand. (2008c). Samoan People in New Zealand: $2006 . \quad$ Retrieved September 2008

Statistics New Zealand. (2010). Pacific Profiles: Samoan - Families and households : 2001. Retrieved 2010, April, from http://www2.stats.govt.nz/domino/external/web/nzstories.nsf/fb471f7feb8c8d5fcc256 af1000fa1c1/f375f35d8c64be01cc256ccb006862f0?OpenDocument

Stevens-Simon, C., Beach, R. K., \& Klerman, L. V. (2001). To be rather than not to be - that is the problem with the questions we ask adolescents about their childbearing intentions. Archives of Pediatrics \& Adolescent Medicine, 155, 1298-1300.

Stewart-Withers, R. (2007). Contesting development: The experience of female-headed households in Samoa. Unpublished PhD Thesis. Massey University.

Stewart-Withers, R., Scheyvens, R., \& Fairbairn-Dunlop, P. (2010). Being a single mum: Pacific Island mothers' positive experience of parenting. Wellington: Families Commission.

Stewart, D. E., Robertson, E., Dennis, C.-L., Grace, S. L., \& Wallington, T. (2003). Postpartum depression: Literature reivew of risk factors and interventions. Toronto: Toronto Public Health.

Stewart, M., \& Hunt, S. C. (Eds.). (2004). Pregnancy, birth, and maternity care: Feminist perspectives. London: Elsevier Butterworth Heinemann.

Tamasese, K., Peteru, C., Waldegrave, C., \& Bush, A. (2005). Ole Taeao Afua, the new morning: a qualitative investigation into Samoan perspectives on mental health and culturally appropriate services. Australian and New Zealand Journal of Psychiatry, 39, 300-309.

Tamasese Ta'isi Efi, T. A. T. (2009). Bioethics and the Samoan indigenous reference. International Social Science Journal, 60(195), 115-124.

Teaiwa, T. (2010). Personal Communication: Re: publication Caring for Ourselves, Vanessa Griffen. Wellington.

Television New Zealand. (2008). Midwife shortage may reach 'crisis'. Auckland: Television New Zealand.

Tiatia, J. (1998). Caught between cultures: A New Zealand-born Pacific Island perspective. Auckland: Christian Research Association.

Tiatia, J. (2003). Reasons to live: NZ-Born Samoan young people's responses to suicidal behaviours. Unpublished PhD Thesis. University of Auckland. 
Tolich, M., \& Davidson, C. (2003). Collecting the data. In C. Davidson \& M. Tolich (Eds.), Social Science Research in New Zealand: Many paths to understanding (2nd ed.). Auckland: Pearson Education New Zealand.

Torkan, B., Parsay, S., Lamyian, M., Kazemnejad, A., \& Montazeri, A. (2009). Postnatal quality of life in women after normal vaginal delivery and caesarean section. BMC Pregnancy and Childbirth, 9(1), 4.

Tupuola, A. M. (1998). 'Adolesence': myth or reality for 'Samoan' women? Beyond the stagelike toward shifting boundaries and identities. Unpublished $\mathrm{PhD}$, Victoria University of Wellington, Wellington.

Tupuola, A. M. (2000). Learning sexuality: Young Samoan Women. In Alison Jones, P. Herda \& T. M. Suaalii (Eds.), Bitter Sweet: Indigenous Women in the Pacific. Dunedin: University of Otago Press.

Tupuola, A. M. (2006). Participatory research, culture and youth identities: An exploration of indigenous, cross-cultural and trans-national methods. Children, Youth and Environments, 16(2), 291-316.

Ungar, M., Brown, M., Liebenberg, L., Othman, R., Kwong, W. M., Armstrong, M., et al. (2007). Unique pathways to resilience across cultures. Adolescence, 42(166), 287310.

Vaughan, G., \& Hogg, M. (2002). Introduction to Social Psychology (3rd ed.). Sydney: Pearson Education.

Waldenstrom, U., Borg, I.-M., Olsson, B., Skold, M., \& Wall, S. (1996). The childbirth experience: A study of 295 new mothers. Birth, 23(3).

Wall, G. (2001). Moral constructions of motherhood in breastfeeding discourse. Gender \& Society, 15(4).

Walsh, S. P., White, K. M., \& Young, R. M. (2008). The Phone Connection: A qualitative exploration of how belongingness and social identification relate to mobilee phone use amongst Australian youth. Journal of Community \& Applied Social Psychology, $19,225-240$.

Watson, J., Hodson, K., Johnson, R., \& Kemp, K. (2002). The maternity experiences of Indigenous women admitted to an acute care setting. Australian Journal of Rural Health(10), 154-160.

Wei, R., \& Lo, V.-H. (2006). Staying connected while on the move: Cell phone use and social connectedness. New Media \& Society, 8(1), 53-72.

Wesley-Smith, T. (1995). Rethinking Pacific Island Studies. Pacific Studies, 18(2), 115-137.

Whitford, H. M., \& Hillan, E. M. (1998). Women's perceptions of birth plans. Midwifery, 14, 248-253.

Williams, K., Lago, L., Lainchbury, A., \& Eagar, K. (2009). Mothers' views of caseload midwifery and the value of continuity of care at an Australian regional hospital. Midwifery.

Williams, L., McCreanor, T., \& Barnes, H. M. (2003). A review of Mental Health promotion literature and analysis of evidence to inform mental health promotion practice in Aotearoa/New Zealand. Wellington: Mental Health Foundation of New Zealand.

World Health Organisation. (2009). Mental health aspects of women's reproductive health: a global review of the literature. Geneva.

York, R., Bhuttarowas, P., \& Brown, L. (1999). The development of nursing in Thailand and its relationship to childbirth practices. The American Journal of Maternal/Child Nursing, 24(3), 145-150. 


\section{GLOSSARY}

$\overline{a i g} a$ - nuclear, immediate or extended family/kin group - descent group

alofa - love, compassion, concern

$a v a$ - respect

fa'aalaolo - reverence

fa'ailoga tama - ritual giving recognition by both āiga to a child.

fa'aSamoa - Samoan way of life; the manner of the Samoans; according to Samoan customs tradition fa'atosaga - a traditional midwife

fofo-Samoan healers

ma 'itaga, ma'ito) - viewed as an illness or a sickness

ma'itagata/ma'itaga - which essentially is to be ill (ma'i) with a new tagata (human being);

pregnancy

milimili - traditional Samoan massage

$p \bar{a} k e h \bar{a}$ - is a Māori term used to describe any peoples of non-Māori or non-Polynesian heritage

papalagi/palagi - of European descent; Western

va fealoa ${ }^{\prime} i$-relationships formed on the basis of trust

\section{Sources:}

(Asiasiga, 2007; Fairbairn-Dunlop, 1998; Ngan-Woo, 1985; Stewart-Withers, 2007) 


\section{APPENDIX ONE}

\section{PARTICIPANT INFORMATION SHEET}

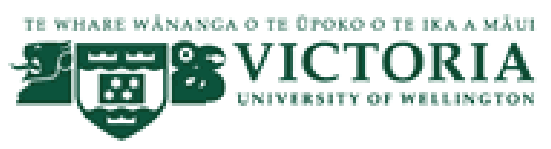

Participant Information Sheet

Title of the project: Factors affecting the health and wellbeing of Samoan women living in New Zealand during late pregnancy and post-birth

I am a Masters student in Social Science Research (Applied) at Victoria University of Wellington. As part of this degree I am undertaking a research project leading to a thesis. The University requires that ethics approval be obtained for research involving human participants. The research project I am undertaking involves exploring factors that affect the health and wellbeing of Samoan women living in New Zealand during late pregnancy and post-birth (being 4-6 months after the birth of their baby). The research will be conducted with Samoan women living in New Zealand. This research will give us an in-depth understanding of the experiences Samoan women have during pregnancy; and the impact of child birth on the emotional wellbeing of women. I am also interested in the range of support systems that Samoan women may have utilised during this time; and some of the coping strategies (if any) that were in place.

The research will give you a chance to discuss ways to help other Samoan women cope through pregnancy and early motherhood. The information you provide will also give us a better understanding of what services or support will help other women and their families and may lead to services that are culturally appropriate and safe.

The individual interviews will take between 1-11/2 hours and it will be arranged at a time and place of your choice. You are welcome to have a support person(s) with you during the interview. There will be two interviews - (1) in your last trimester of pregnancy; and (2) 4-6 months after you have given birth.

The information you provide will be kept confidential, that means you will not be able to be identified, and the information will be securely stored. All results will be presented in an aggregated form and every attempt will be taken to maintain confidentiality of participants identity. However, as participants will be drawn from a small community there is always a very slight chance that you may be recognized by someone who knows you well. Participation in this research is voluntary and you can withdraw from this research up to two weeks after the interview.

About the researcher: My name is Marianna Churchward, I was born in New Zealand, both my parents are of Samoan descent, I identify as New Zealand-born Samoan. I am the mother of three children. My contact details are: Health Services Research Centre, Victoria University of Wellington, PO Box 600, Wellington. Phone: (04) 463-6583. Email: marianna.churchward@ vuw.ac.nz/If you have any further questions please contact myself or my supervisor, Dr Teresia Teaiwa, Va'aomanu Pasifika, Pacific Studies, Victoria University of Wellington. Phone: (04) 4635827. 


\section{APPENDIX TWO \\ PARTICIPANT CONSENT FORM \\ -Interview One and Interview Two -}

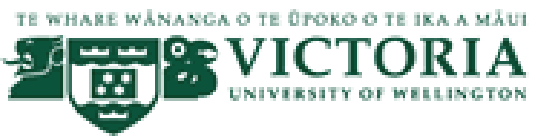

\section{CONSENT FORM}

Title of the project: Factors affecting the health and wellbeing of Samoan women living in New Zealand during late pregnancy and post-birth

I have the read the information sheet. I have been given the opportunity to ask any questions I may have and these questions have been answered by my satisfaction.

口 I understand there will be two interviews and my agreement to take part in both.

口 I agree to be interviewed for this research project by Marianna Churchward.

口 I agree to the tape-recording of the interview and understand that copies of the tapes will be kept in a locked cabinet, and destroyed 3 years after the completion of the research. (NB: This is a criteria for participating in this interview).

口 I understand that tapes will be heard and transcripts will be read only by Marianna Churchward.

口 I understand that Marianna Churchward and an academic supervisor will have access to the tapes and transcripts.

口 I understand that I may withdraw information from the study up to two weeks after the interview.

口 I understand that all the information I provide will be kept confidential and no identifying information about me will be published in the final report.

口 I understand that the data I provide will not be used for any other purpose or released to others without my written consent.

口 If at any time during the interview I am feeling uncomfortable or distressed to continue the interview, I will let Marianna know immediately.

$\square \quad$ I have been given information of agencies to call if I feel distressed after the interview

$\square \quad$ I would like to receive a summary of the final report

Signed:

Interview $1 / 2$ (please circle)

Name: Date: 


\title{
APPENDIX THREE \\ INTERVIEW SCHEDULE
}

\author{
- Interview One -
}

\section{Interview Schedule 1}

Title of the project: Factors affecting the health and wellbeing of Samoan women living in New Zealand during late pregnancy and post-birth

Phase 1: Last trimester of pregnancy

The research is about women's experience of pregnancy and motherhood. It also explores factors that have affected and or contributed to that experience. The broad questions are to guide the researcher to ensure specific areas will be explored. It is a semi-structured interview that intends to allow the emergence of themes that are constructed by the women themselves.

\begin{tabular}{|c|c|}
\hline Broad Questions & Prompts \\
\hline Self & $\begin{array}{l}\text { Tell me a little about yourself ... e.g. hobbies, family, working etc. } \\
\text { Who do you live with? } \\
\text { Are you in paid employment (do you work) }\end{array}$ \\
\hline Knowledge of your body & $\begin{array}{l}\text { How important is it for women to be knowledgeable about their bodies? } \\
\text { How important is it for Samoan women to be knowledgeable about their bodies? }\end{array}$ \\
\hline Decision to have a baby & $\begin{array}{l}\text { What were some of the reasons behind the decision to have a baby? Was it planned? } \\
\text { Who was involved in that decision-making process? }\end{array}$ \\
\hline Getting pregnant & Were there any issues about getting pregnancy? \\
\hline Pregnancy & $\begin{array}{l}\text { Lets talk about your pregnancy, what was it like physically, emotionally, spiritually, } \\
\text { socially. Explore the experience. } \\
\text { (If participant describes a difficult pregnancy) what strategies did you adopt to get } \\
\text { you through? } \\
\text { (If participant describes a relatively easy pregnancy) what do you think contributed } \\
\text { to your 'easy' pregnancy? } \\
\text { Do you have a midwive? When did you register with your midwive? Did you go to } \\
\text { antenatal classes? }\end{array}$ \\
\hline Cultural/traditional beliefs & $\begin{array}{l}\text { Do you think your beliefs about pregnancy and motherhood are specific to the } \\
\text { Samoan culture? (explore) }\end{array}$ \\
\hline Illness / Wellness & Do you think pregnancy is an 'illness' or a 'wellness' \\
\hline History of feeling sad & $\begin{array}{l}\text { In the past have you suffered from general depression? } \\
\text { What do you think 'postnatal' depression means? } \\
\text { Describe what it means to have 'postnatal depression' }\end{array}$ \\
\hline $\begin{array}{l}\text { Support systems } \\
\text { (FORMAL AND INFORMAL) }\end{array}$ & $\begin{array}{l}\text { What or who are your support systems? (explore) } \\
\text { (informational eg advice) } \\
\text { (instrumental aid assisting with tasks) } \\
\text { (emotional) } \\
\text { Specific support needs - who helped? What helped? } \\
\text { Specific support needs - who hindered? What hindered? } \\
\text { Did you seek help? What were some of the barriers? }\end{array}$ \\
\hline Expectations of labour & $\begin{array}{l}\text { What are your expectations of giving birth? } \\
\text { Hospital/home birth? What do you expect to happen in hospital/home during your } \\
\text { labour? }\end{array}$ \\
\hline Expectations of motherhood & $\begin{array}{l}\text { Thinking about the } 4-6 \text { months after baby is born, describe what you think your life } \\
\text { is going to be like? How do you think you will feel emotionally, spiritually, } \\
\text { physically, socially etc. }\end{array}$ \\
\hline
\end{tabular}




\title{
APPENDIX FOUR \\ INTERVIEW SCHEDULE
}

\author{
- Interview Two -
}

\author{
Interview Schedule 2
}

Title of the project: Factors affecting the health and wellbeing of Samoan women living in New Zealand during late pregnancy and post-birth

Phase 2: 6-12 months after birth

The research is about women's experience of pregnancy and motherhood. It also explores factors that have affected and or contributed to that experience. The broad questions are to guide the researcher to ensure specific areas will be explored. It is a semi-structured interview that intends to allow the emergence of themes that are constructed by the women themselves.

\begin{tabular}{|c|c|}
\hline Broad Questions & Prompts \\
\hline Self & How have you been since we last met? \\
\hline Experience of labour & $\begin{array}{l}\text { Talk me through your birth? } \\
-\quad \text { How did you know you were in labour? } \\
-\quad \text { hospital/home? } \\
-\quad \text { Any difficulties? } \\
-\quad \text { Who was with you at the birth? } \\
\text { - } \quad \text { How important was it to have those people there? } \\
\text { - Did they help with your labour? Or not? } \\
\text { How long did you stay in hospital? } \\
\text { - } \quad \text { was that enough time for you? } \\
\text { - } \quad \text { Did you want to stay longer/shorter? } \\
\quad \text { What were the sorts of things you really needed help with? Did you get that } \\
\quad \text { help? (for e.g. from the nurses, midwives) } \\
\text { How did you feel emotionally after giving birth? } \\
\text { What language did you use during your birth? (Samoan/English or both) } \\
\text { What about physically? } \\
\text { Who helped deliver the baby? } \\
\text { If it was a caesarean explore the experience - the expectation of a vaginal birth yet } \\
\text { having a caesarean ... any problem? } \\
\text { THINKING BACK TO YOUR EXPECTATIONS, HOW DIFFERENT/SIMILAR } \\
\text { WAS THIS EXPERIENCE TO WHAT YOU EXPECTED? } \\
\text { - } \quad \text { if there was a marked difference, how did you cope through this difference? }\end{array}$ \\
\hline $\begin{array}{l}\text { Experience of motherhood } \\
\text { At home (FIRST WEEK) } \\
\text { Some mothers feel quite nervous } \\
\text { about the responsibility that lies } \\
\text { ahead whereas others feel quite } \\
\text { confident. }\end{array}$ & $\begin{array}{l}\text { How were you feeling when you left the hospital to go home? } \\
\text { Was it your decision to leave the hospital? How did that make you feel? } \\
\text { Who did you go home with? } \\
\text { Who was with you? } \\
\text { Was there anyone at home waiting for you? If so, who? } \\
\text { Who are the people that have helped you during this time? } \\
\text { Who have you most relied on? }\end{array}$ \\
\hline
\end{tabular}




\begin{tabular}{|c|c|}
\hline & $\begin{array}{l}\text { THINKING BACK TO YOUR EXPECTATIONS, HOW DIFFERENT/SIMILAR } \\
\text { WAS THIS EXPERIENCE TO WHAT YOU EXPECTED? }\end{array}$ \\
\hline UPTO 4 MONTHS & $\begin{array}{l}\text { Describe how you felt during this time? } \\
\text { Describe as best you can what a typical day during this period would look like? }\end{array}$ \\
\hline At home (4-6 months) & 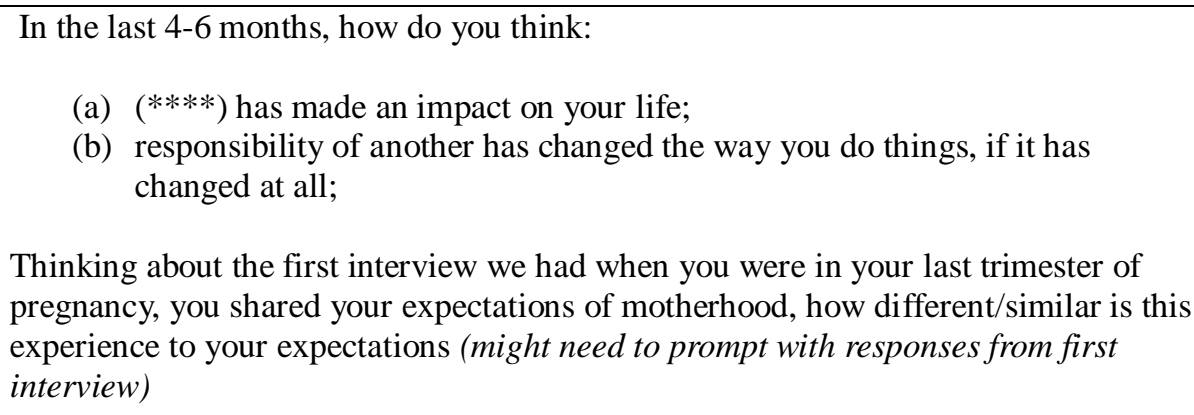 \\
\hline EMPLOYMENT & $\begin{array}{l}\text { Are you on maternity leave - did you return to work? } \\
\text { Is it the same work you were involved in before you got pregnant? } \\
\text { Did you want to go back to work? } \\
\text { Did your husband take maternity leave from work? How long? }\end{array}$ \\
\hline $\begin{array}{l}\text { At home (6-12 months) } \\
\text { SELF-CARE }\end{array}$ & $\begin{array}{l}\text { Do you feel okay to ask for help? } \\
\text { Who are you most likely to ask? How would you ask? } \\
\text { What help would you ask for? } \\
\text { How important is it for you to have time to yourself? }\end{array}$ \\
\hline Cultural/traditional beliefs & $\begin{array}{l}\text { What is your understanding of traditional Samoan beliefs around birth and } \\
\text { motherhood? } \\
\text { What have you heard from your mother, aunty, grandmother, sister? }\end{array}$ \\
\hline Support systems & $\begin{array}{l}\text { FORMAL AND INFORMAL SUPPORT SYSTEMS: } \\
\text { PRACTICAL - housework, preparing meals, helping with the baby } \\
\text { SOCIAL AND EMOTIONAL - shoulder to cry on, someone to confide in, providing } \\
\text { reassurance } \\
\text { INFORMATION OR ADVICE - helping with breasfeeding, answering questions } \\
\text { What or who are your support systems? (explore) } \\
\text { Specific support needs - who helped? What helped? } \\
\text { Specific support needs - who hindered? What hindered? } \\
\text { Did you seek help? What were some of the barriers? } \\
\text { EXAMPLE OF SUPPORTERS: } \\
\text { Partner } \\
\text { Parents/Parents-in-Law/Parents of partner } \\
\text { Grandparents } \\
\text { Siblings/Siblings-in-law/siblings of partner } \\
\text { Other close relatives: aunties, uncles, cousins,extended family } \\
\text { Friends/neighbours } \\
\text { Midwife } \\
\text { Plunket Line O8OO number } \\
\text { Plunket Nurse } \\
\text { New mother support groups } \\
\text { PIN groups } \\
\text { Parent centrelother antenatal groups } \\
\text { GP/paediatrician/specialist } \\
\text { Television programmes } \\
\text { Books/magazines }\end{array}$ \\
\hline
\end{tabular}




\begin{tabular}{|l|l|}
\hline Advice & Other $(s)$ \\
\hline \multirow{5}{*}{$\begin{array}{l}\text { If you had the opportunity to talk with a first-time Samoan mother, what are some of } \\
\text { the things that you were told by your support network that are valuable enough to } \\
\text { pass on? }\end{array}$} \\
$\begin{array}{l}\text { What are some of the things you experienced that you think women would benefit } \\
\text { from knowing? } \\
\text { What are some not so good things that you experienced or were told that women } \\
\text { should know but not worry about? (explore) } \\
\text { What are some positive things that you experienced during pregnancy or post-birth } \\
\text { that you think would be beneficial to share with others? (explore) }\end{array}$ \\
\hline DEMOGRAPHICS & $\begin{array}{l}\text { Age: } \\
\text { Partner: } \\
\text { Paid employment before baby was born? } \\
\text { Paid employment now? } \\
\text { When did you go back to work? } \\
\text { What sort of work? } \\
\text { Partner working? } \\
\text { Living circumstances }\end{array}$ \\
\hline
\end{tabular}

